Aus der Abteilung Anaesthesiologie

(Prof. Dr. med. M. Quintel)

im Zentrum Anaesthesiologie, Rettungs- und Intensivmedizin

der Medizinischen Fakultät der Universität Göttingen

\title{
Evaluierung, Validierung und Anwendung eines Scores zur \\ Stratifizierung des Risikos akuter Nierenfunktionsstörungen nach kardiochirurgischen Operationen
}

\author{
INAUGURAL - DISSERTATION \\ zur Erlangung des Doktorgrades \\ der Medizinischen Fakultät der \\ Georg-August-Universität zu Göttingen
}

vorgelegt von

Anna Julienne Wetz

aus Lich 
Dek a n:

I. Berichterstatter:

II. Berichterstatter/in:

III. Berichterstatter/in:

Tag der mündlichen Prüfung:
Prof. Dr. rer. nat. H. K. Kroemer

Prof. Dr. med. A. Bräuer 


\section{INHALTSVERZEICHNIS}

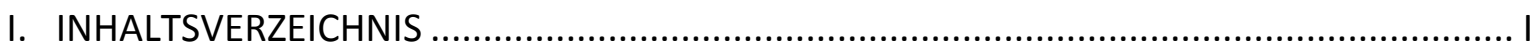

II. ABKÜRZUNGEN, ABBILDUNGEN UND TABELLEN ................................................. VI

II.1 Liste der verwendeten Abkürzungen............................................................. VI

II.2 Abbildungsverzeichnis ...................................................................................... VII

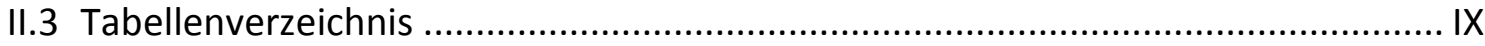

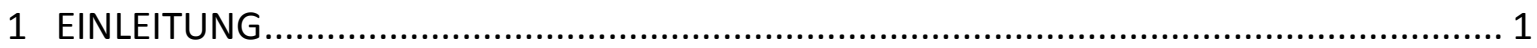

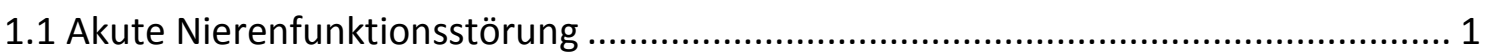

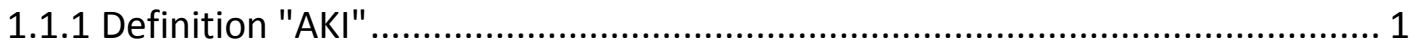

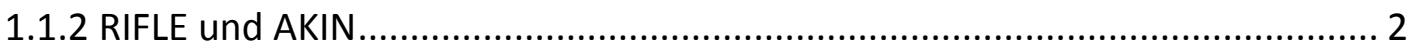

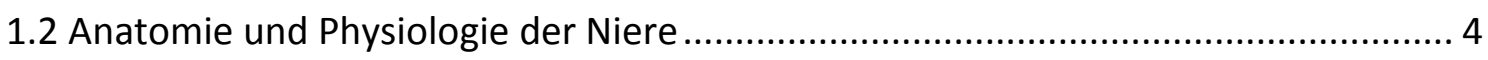

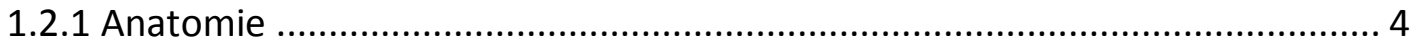

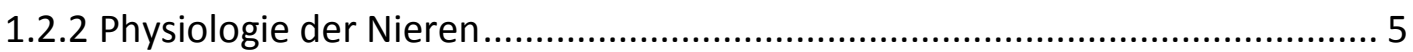

1.2.2.1 Nierendurchblutung und glomeruläre Filtrationsrate ....................... 5

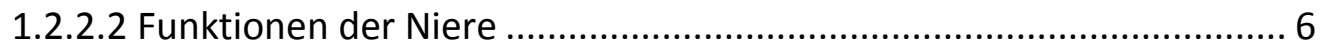

1.3 Einteilung, Ätiologie und Pathophysiologie des ANV .............................................. 8

1.3.1 Klassische Einteilung: Prä-, intra- und postrenal .......................................... 8

1.3.1.1 Prärenales Nierenversagen .............................................................. 9

1.3.1.1.1 Pathophysiologie des zirkulatorisch-ischämisch-bedingten prärenalen Nierenversagens ........................................ 10

1.3.1.2 Intrarenales Nierenversagen ......................................................... 11

1.3.1.3 Postrenales Nierenversagen ........................................................... 11

1.3.2 Häufigkeiten der unterschiedlichen Formen ............................................ 12

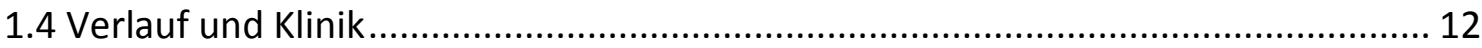

1.5 Postoperatives AKI nach Kardiochirurgie ............................................................. 13

1.5.1 Verwendung der Herz-Lungen-Maschine ................................................ 13

1.5.2 Inzidenz und Auswirkungen auf Mortalität ................................................. 14

1.5.3 Ursachen für die hohe Inzidenz eines AKI nach kardiochirurgischen

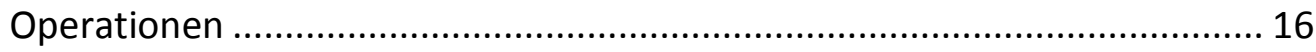

1.5.3.1 Renale Hypoperfusionsschädigung .............................................. 17

1.5.3.2 Oxido-inflammatorischer Stress.................................................... 18 
1.5.3.3 Hämolyse induziert Nierenschädigung .......................................... 18

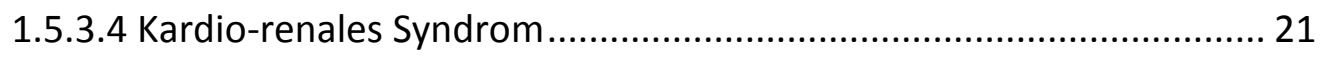

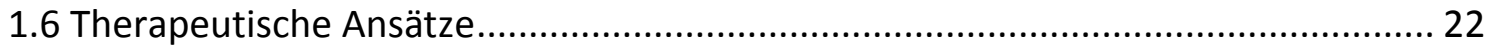

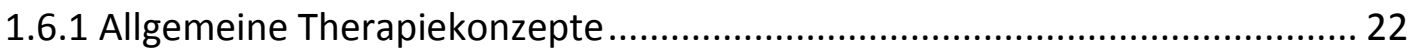

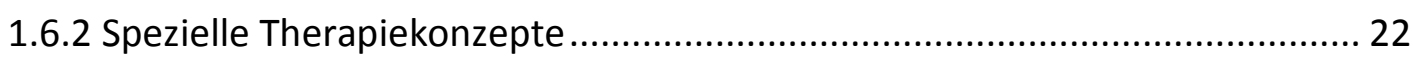

1.6.2.1 Therapie der Grunderkrankung ................................................... 22

1.6.2.2 Sekundär präventive Maßnahmen............................................... 23

1.6.2.2.1 Volumentherapie ......................................................... 23

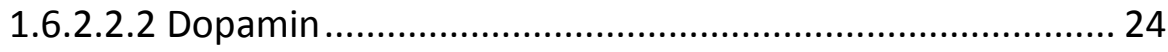

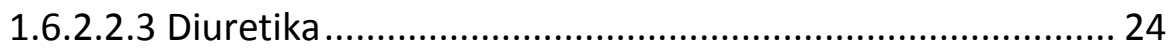

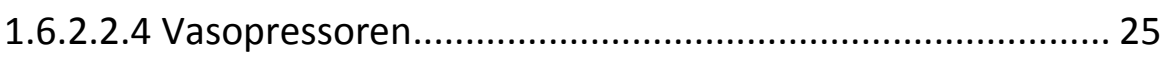

1.6.2.2.5 Vasodilatatoren ........................................................... 25

1.6.2.2.6 Hormonelle Therapie ................................................... 25

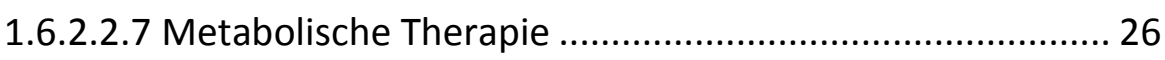

1.6.2.2.8 Nierenersatztherapie ................................................... 26

1.6.3 Spezielle Therapiekonzepte in der Kardiochirurgie................................... 26

1.6.4 Prävention des AKI durch Gabe von Natriumbicarbonat .............................. 28

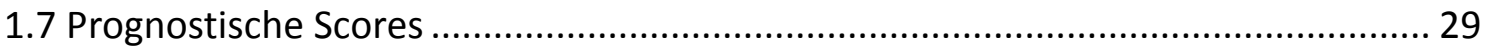

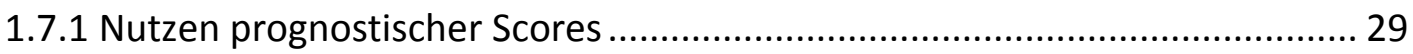

1.7.2 Charakteristika von prognostischen Scores.............................................. 29

1.7.3 Bislang publizierte prognostische Scores ................................................. 30

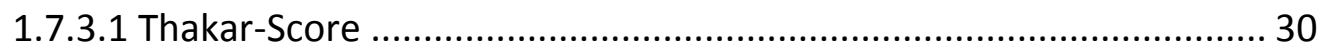

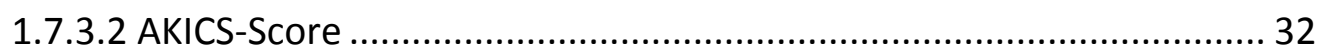

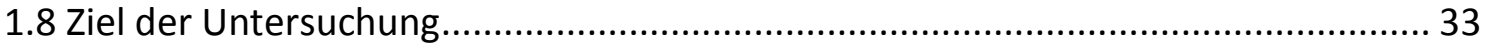

1.8.1 Validität des Thakar-Scores in einem Göttinger Kollektiv unter Verwendung

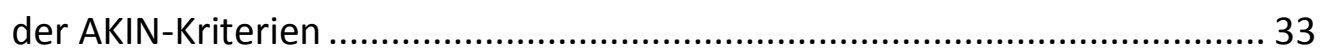

1.8.2 Modifikation des Thakar-Scores durch Re-Evaluation ................................ 34

1.8.3 Modifikation des Thakar-Scores durch Hinzunahme intraoperativer

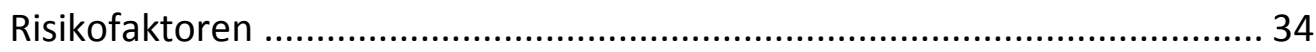

1.8.4 Auswirkung einer Therapie mit Natriumbicarbonat ..................................... 35

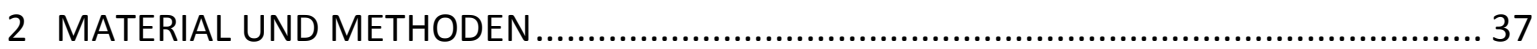

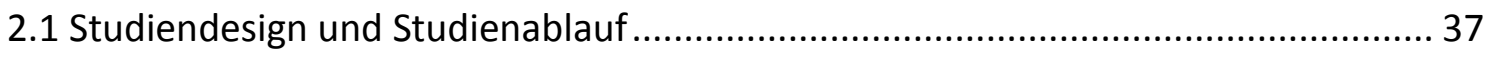




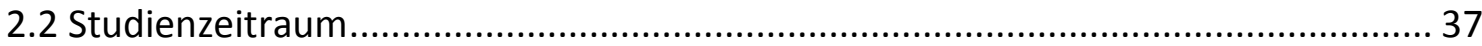

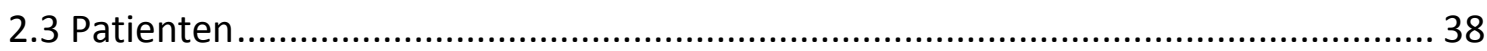

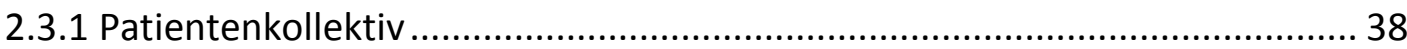

2.3.2 Patienteninformation und Einwilligungserklärung..................................... 38

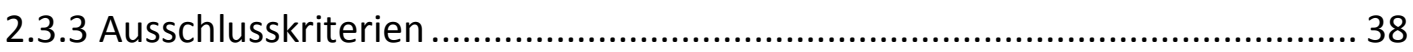

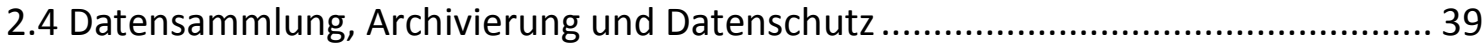

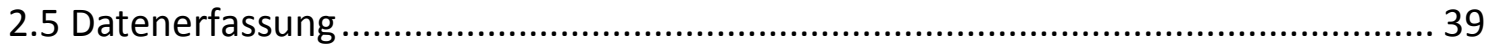

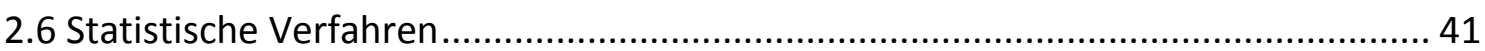

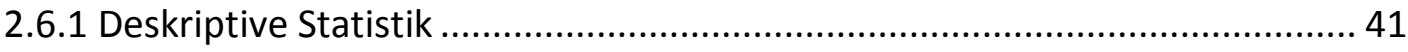

2.6.2 Überprüfung der Validität des Thakar-Scores am Göttinger Kollektiv........... 42

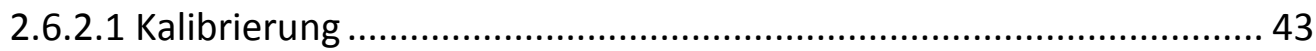

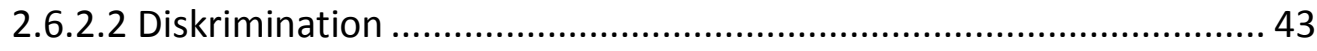

2.6.2.2.1 Sensitivität.................................................................. 43

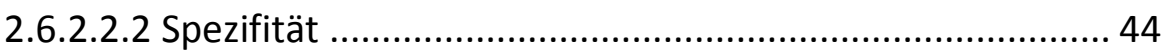

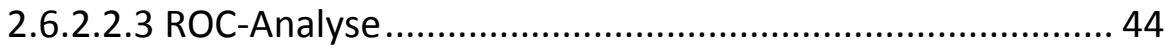

2.6.3 Re-Evaluation des Thakar-Scores am Göttinger Kollektiv ........................... 46

2.6.3.1 Monovariate Analyse ...................................................................... 46

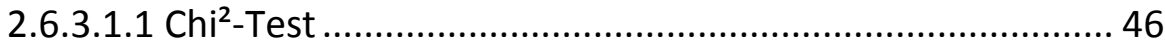

2.6.3.1.2 Normalverteilung der kontinuierlichen Variablen ............ 47

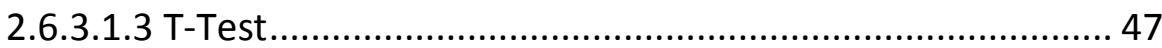

2.6.3.1.4 Mann-Whitney-U-Test ................................................... 47

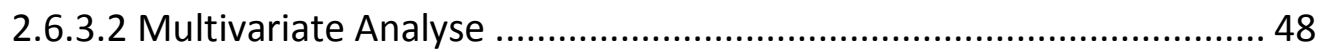

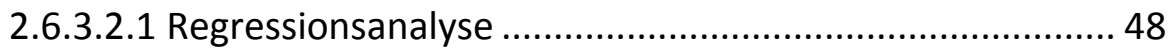

2.6.3.2.2 Logistische Regression ................................................. 49

2.6.3.2.3 Regressionskoeffizient ................................................... 49

2.6.3.2.4 Bildung der Odds Ratio .................................................. 50

2.6.3.2.5 Bildung der Score-Punkte ............................................ 51

2.6.3.2.6 Validierung der modifizierten Scores.............................. 52

2.6.4 Einfluss der Natriumbicarbonat-Therapie auf die postoperative

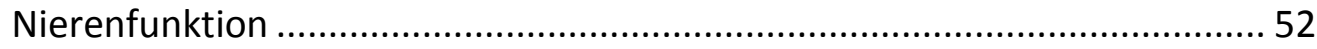

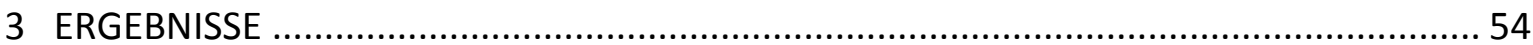

3.1 Demographie des Patientenkollektivs ............................................................. 54 
3.2 Häufigkeitsverteilung der untersuchten Risikofaktoren .................................... 54

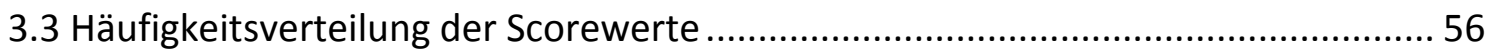

3.4 Inzidenz des AKI im Göttinger Kollektiv ............................................................ 58

3.4.1 Häufigkeitsverteilung der AKIN-Stadien .................................................... 58

3.4.2 Inzidenzen der akuten Nierenschädigung in Abhängigkeit der Scorepunkte 58

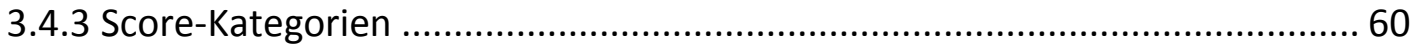

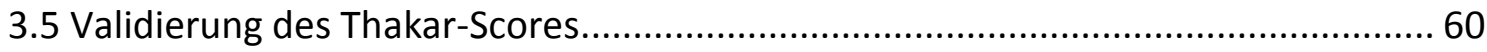

3.5.1 Kalibrierung des Thakar-Scores bei Anwendung am Göttinger Kollektiv....... 60

3.5.2 Diskrimination des Thakar-Scores bei Anwendung am Göttinger Kollektiv ...61

3.6 Re-Evaluierung des Thakar-Scores am Göttinger Kollektiv ................................... 62

3.6.1 Univariate Analyse der Risikofaktoren .................................................. 62

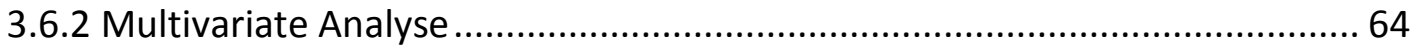

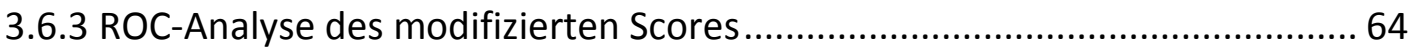

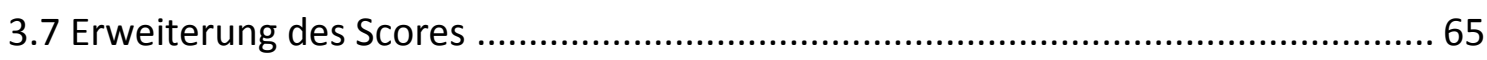

3.7.1 Univariate Analyse der kontinuierlichen Risikofaktoren ............................... 65

3.7.2 Dichotomisierung der kontinuierlichen Risikofaktoren ............................. 66

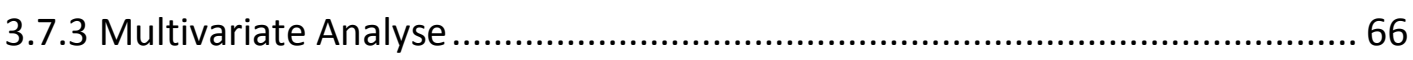

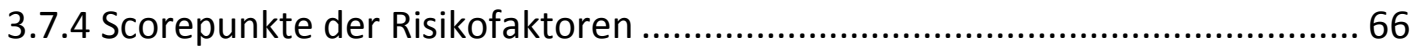

3.7.5 Diskriminationsfähigkeit des erweiterten Scores ...................................... 67

3.8 Zusammenfassung der Ergebnisse der Evaluierung und Validierung ......................68 68

3.9 Untersuchung der Auswirkung der Natriumbicarbonattherapie .............................68

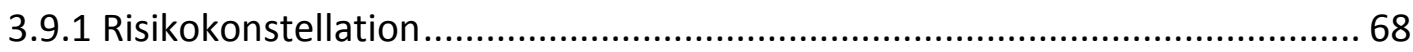

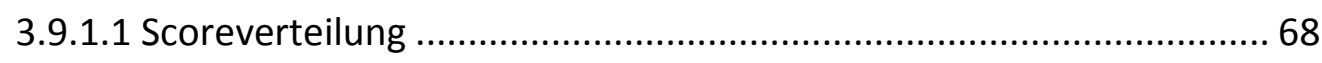

3.9.1.2 Freie Serumhämoglobinwerte .................................................... 69

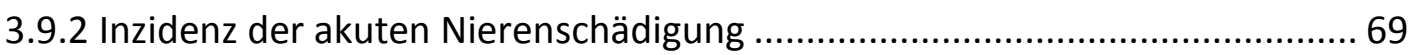

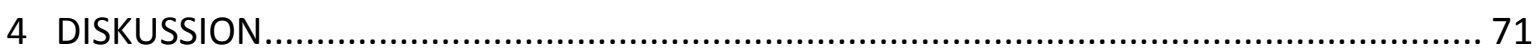

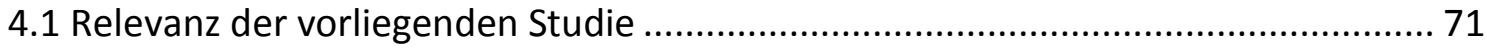

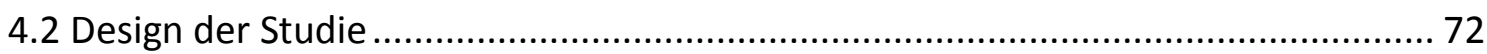

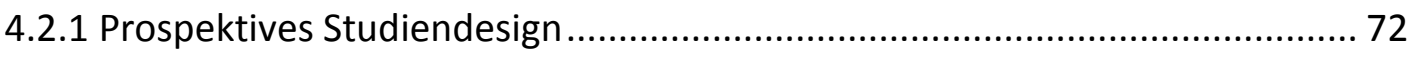

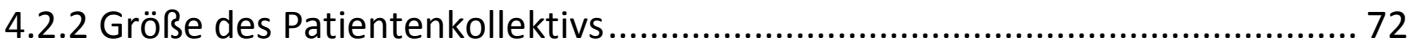

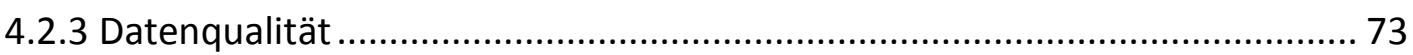

4.2.4 Endpunkt: Definition der akuten Nierenschädigung .................................. 74 


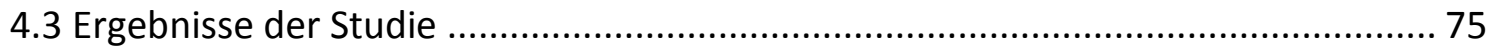

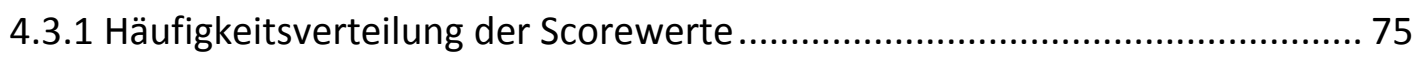

4.3.2 Häufigkeitsverteilung der Risikofaktoren ................................................. 76

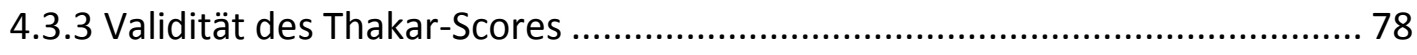

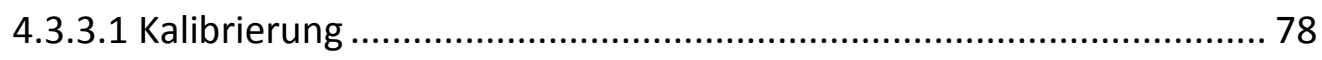

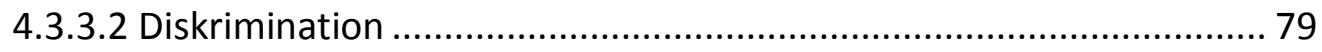

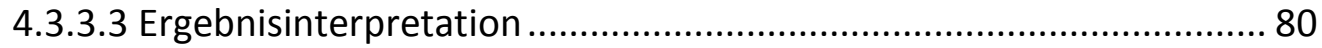

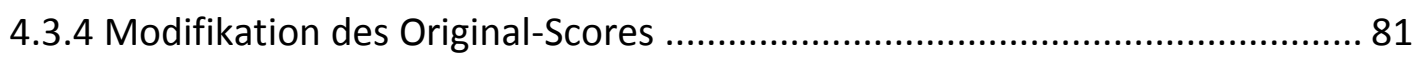

4.3.4.1 Neuberechnung des Original-Scores............................................ 81

4.3.4.2 Erweiterung um intraoperative Risikofaktoren ............................. 83

4.3.5 Einfluss von Natriumbicarbonat auf die postoperative Nierenfunktion ........ 84

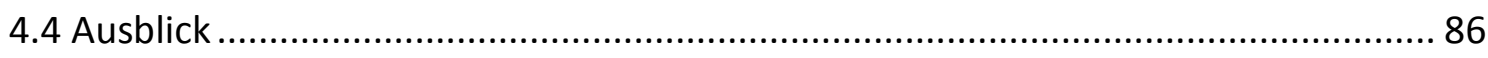

4.4.1 Validierung anderer Scores, Vergleich von Scoring-Systemen...................... 86

4.4.2 Scores mit Berücksichtigung der AKIN-Stadien ......................................... 87

4.4.3 Anwendung und Auswirkung eines Scores.................................................. 88

4.4.4 Etablierte präventive Maßnahmen .......................................................... 90

4.4.5 Experimentelle präventive Maßnahmen ................................................... 91

4.4.5.1 Haptoglobin ........................................................................... 91

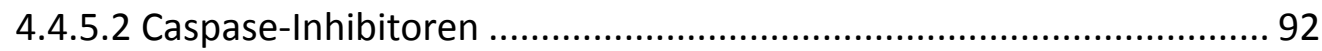

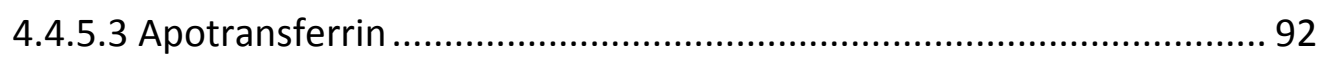

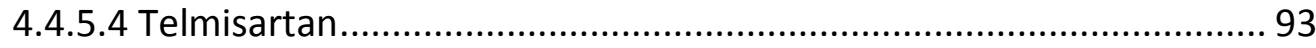

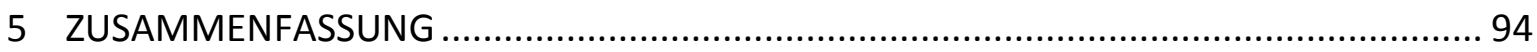

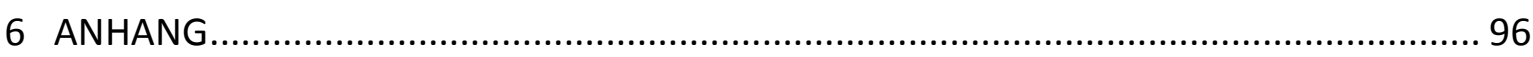

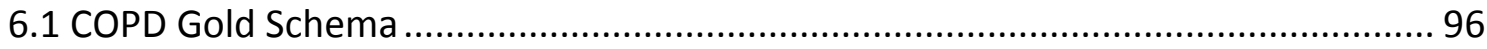

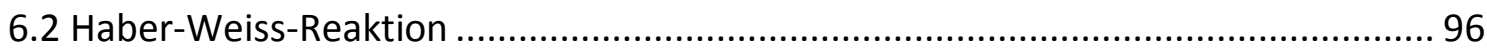

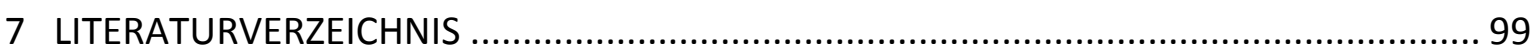




\section{Abkürzungen, Abbildungen und Tabellen}

\section{II.1 Liste der verwendeten Abkürzungen}

\begin{tabular}{|c|c|}
\hline $\mathrm{ACB}$ & Aortokoronarer Bypass \\
\hline ACE & Angiotensin Converting Enzyme \\
\hline ADQI & Acute Dialysis Quality Initiative \\
\hline AKI & Acute Kidney Injury (Akute Nierenschädigung) \\
\hline AKIN & Acute Kidney Injury Network (Klassifikation) \\
\hline ANV & Akutes Nierenversagen \\
\hline ARDS & Acute Respiratory Distress Syndrome \\
\hline ATP & Adenosintriphosphat \\
\hline$A \cup C$ & Area under the curve \\
\hline cAMP & Zyklisches Adenosinmonophosphat \\
\hline $\mathrm{cm} \mathrm{H} \mathrm{H}_{2} \mathrm{O}$ & Zentimeter Wassersäule \\
\hline $\mathrm{CO}$ & Cardiac Output \\
\hline $\mathrm{CO}_{2}$ & Kohlendioxid \\
\hline COPD & Chronisch obstruktive Lungenerkrankung \\
\hline CRS & Kardio-renales Syndrom \\
\hline CVVH & Kontinuierlich veno-venöse Hämofiltration \\
\hline DIC & Disseminierte intravasale Gerinnung \\
\hline EPO & Erythropoetin \\
\hline ESICM & European Society of Intensive Care Medicine \\
\hline ESRD & End-Stage-Nierenerkrankung \\
\hline $\mathrm{Fe}^{2+}$ & Eisen-Zwei-Oxid \\
\hline FFP & Fresh-Frozen-Plasma \\
\hline GFR & Glomeruläre Filtrationsrate \\
\hline ggfs. & gegebenenfalls \\
\hline $\mathrm{h}$ & Stunde \\
\hline HES & Hydroxethylstärke \\
\hline $\mathrm{H}_{2} \mathrm{O}$ & Wasser \\
\hline HLM & Herz-Lungen-Maschine \\
\hline HUS & Hämolytisch urämisches Syndrom \\
\hline IABP & Intraaortale Ballonpumpe \\
\hline IL-6 & Interleukin 6 \\
\hline LVEF & Linksventrikuläre Ejektionsfraktion \\
\hline MAP & Mittlerer arterieller Druck \\
\hline $\mathrm{mg} / \mathrm{dl}$ & Milligramm pro Deziliter \\
\hline $\min$ & Minute \\
\hline $\mathrm{ml}$ & Milliliter \\
\hline $\mathrm{mmHg}$ & Millimeter Quecksilbersäule \\
\hline
\end{tabular}




$\begin{array}{ll}\mathrm{NaHCO}_{3} & \text { Natriumbicarbonat } \\ \text { NSAR } & \text { Nicht Steroidale Antirheumatika } \\ \text { NYHA } & \text { New York Heart Association (Herzinsuffizienz-Klassifikation) } \\ \text { OP } & \text { Operation } \\ \text { RAA } & \text { Renin-Angiotensin-Aldosteron-System } \\ \text { RIFLE } & \text { Risk Injury Failure Loss Endstage } \\ \text { SIRS } & \text { Systemic Inflammatory Response Syndrome } \\ \text { TNF } & \text { Tumornekrosefaktor } \\ \text { TTP } & \text { Thrombotisch thrombozytopenische Purpura } \\ \text { Vs. } & \text { versus } \\ \text { ZVD } & \text { Zentralvenöser Druck }\end{array}$




\section{II.2 Abbildungsverzeichnis}

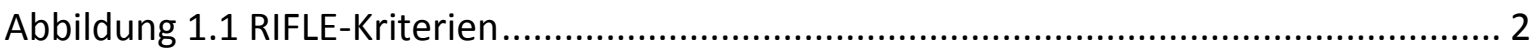

Abbildung 1.2 Ätiologische Einteilung mit den wichtigsten Ursachen des akuten

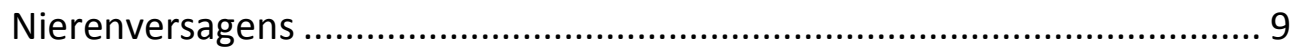

Abbildung 1.3 Schema der extrakorporalen Zirkulation .............................................. 14

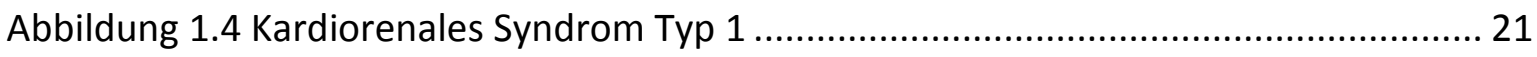

Abbildung 3.1 Histogramm der Scoreverteilung .................................................................. 57

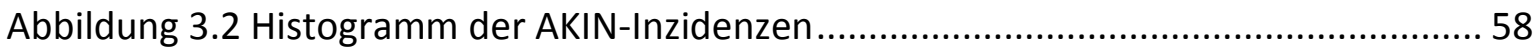

Abbildung 3.3 AKI in Abhängigkeit der Scorewerte .................................................... 59

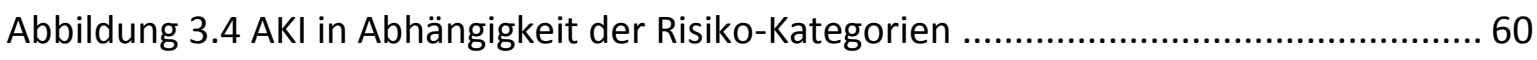

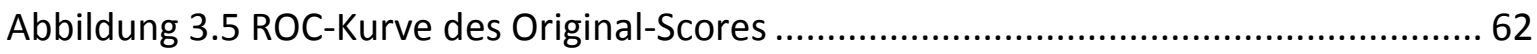

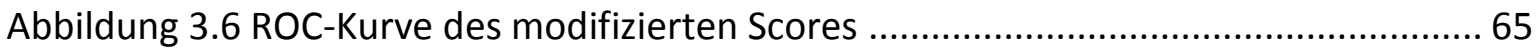

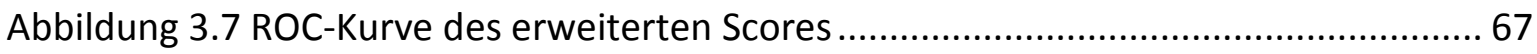

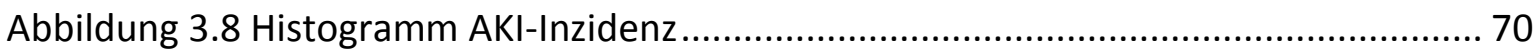




\section{II.3 Tabellenverzeichnis}

Tabelle 1.1 AKIN-Klassifikationssystem der akuten Nierenschädigung ............................. 4

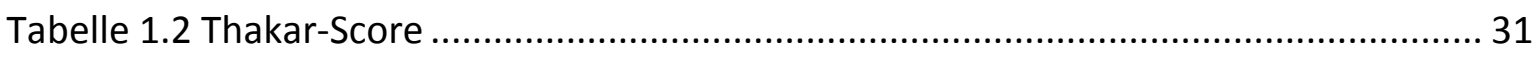

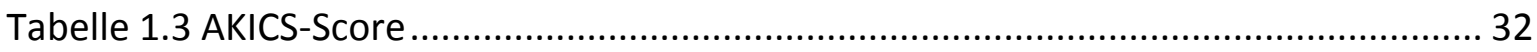

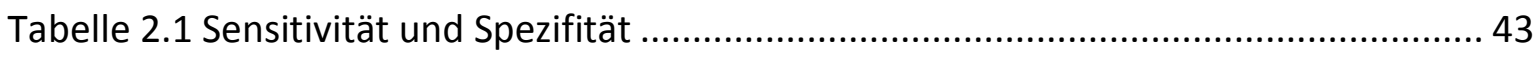

Tabelle 3.1 Verteilung der Risikofaktoren in abnehmender Häufigkeit............................. 55

Tabelle 3.2 Häufigkeitsverteilung der Scorewerte (in allen AKIN-Gruppen)....................... 56

Tabelle 3.3 Häufigkeitsverteilung der AKIN-Stadien .................................................... 58

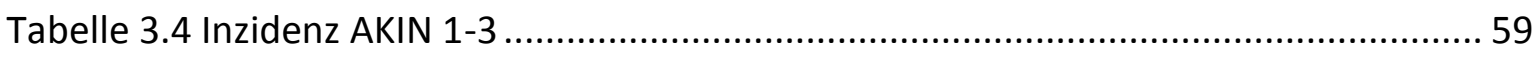

Tabelle 3.5 AKI-Inzidenzen im Göttinger Kollektiv im Vergleich zu Cleveland.................... 61

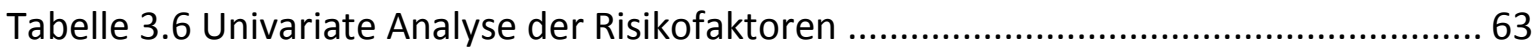

Tabelle 3.7 Regressionskoeffizienten, Odds Ratios und Scorepunkte ................................6 64

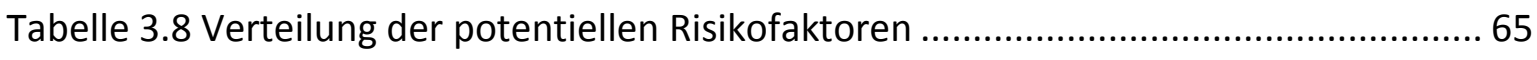

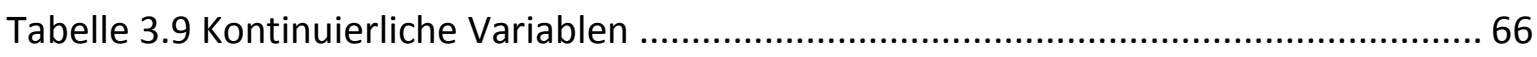

Tabelle 3.10 Regressionskoeffizienten, Odds Ratios und Scorepunkte des erweiterten

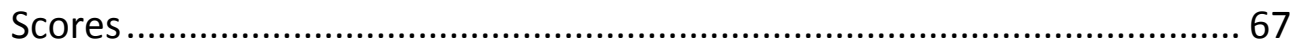

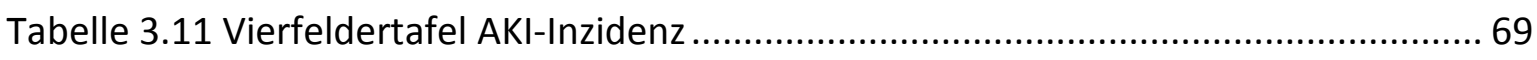

Tabelle 4.1 Häufigkeitsverteilung der Risikofaktoren in Göttingen und Cleveland ............ 76 


\section{EINLEITUNG}

Nierenfunktionsstörungen gehören zu den häufigsten Organdysfunktionen in der Intensivmedizin (de-Mendona et al. 2000). Dabei zählen kardiochirurgische Operationen zu den häufigsten Ursachen eines postoperativen akuten Nierenversagens (Dasta et al. 2008), was nicht nur weitreichende medizinische, sondern auch ökonomische Konsequenzen (Mangano et al. 1998) trägt. Einen wichtigen Bestandteil der gezielt eingesetzten Prävention stellen Scoring-Systeme dar, die das individuelle Risiko für das Auftreten einer akuten Nierenschädigung ermitteln (Parolari et al. 2012). Die vorliegende Arbeit untersucht die Anwendbarkeit eines bereits mehrfach validierten Scores, die Auswirkungen von Modifikationen sowie seine gezielte Anwendung bei der Beurteilung eines neuen prophylaktischen Therapieansatzes.

\subsection{Akute Nierenfunktionsstörung}

\subsubsection{Definition "AKI"}

AKI stellt ein Akronym für Acute Kidney Injury dar, in der deutschen Literatur als akute Nierenschädigung übersetzt. Es findet sich eine Vielzahl verschiedener Formulierungen zur Definition des akuten Nierenversagens selbst. Das akute Nierenversagen ist gekennzeichnet durch eine akut einsetzende, rasche Abnahme der in- und exkretorischen Nierenfunktion, die über Tage anhält und prinzipiell reversibel ist. Es kommt zur Retention harnpflichtiger Substanzen und zur Störung des Flüssigkeits-, Elektrolyt- und SäureBasen-Haushalts.

Leitsymptom ist das Versiegen der Harnsekretion mit Oligo- oder Anurie und einem Anstieg des Serumkreatinins (Herold 2012). 


\subsubsection{RIFLE und AKIN}

Lange Zeit gab es die Notwendigkeit einer einheitlichen Definition und eines allgemeingültigen Klassifikationssystems für das Syndrom der akuten Nierenschädigung. Das Hauptziel bestand darin, Standards für dieses häufige intensivmedizinische Syndrom einzuführen, wie sie für andere intensivmedizinische Syndrome wie beispielsweise Sepsis und ARDS vorhanden sind. Solange keine einheitlichen Kriterien zur Diagnose vorlagen, konnten auch keine einheitlichen Behandlungsempfehlungen ausgesprochen werden. So erarbeitete 2004 die Expertengruppe Acute Dialysis Quality Initiative (ADQI) die RIFLEKriterien. Ein Akronym für drei Schweregradstadien: Risiko „risk“, Schädigung „injury“ und Versagen „failure“ und zwei Verlaufsformen: persistierendes akutes Nierenversagen „loss" und „End-Stage“-Nierenerkrankung (ESRD). Es handelt sich um ein objektives und einfach durchführbares Klassifikationssystem durch Betrachten von folgenden Parametern: der Diuresemenge, des relativen Kreatininanstiegs (im Stadium F auch als absoluter Wert) und der GFR-Einschränkung (Bellomo et al. 2004).

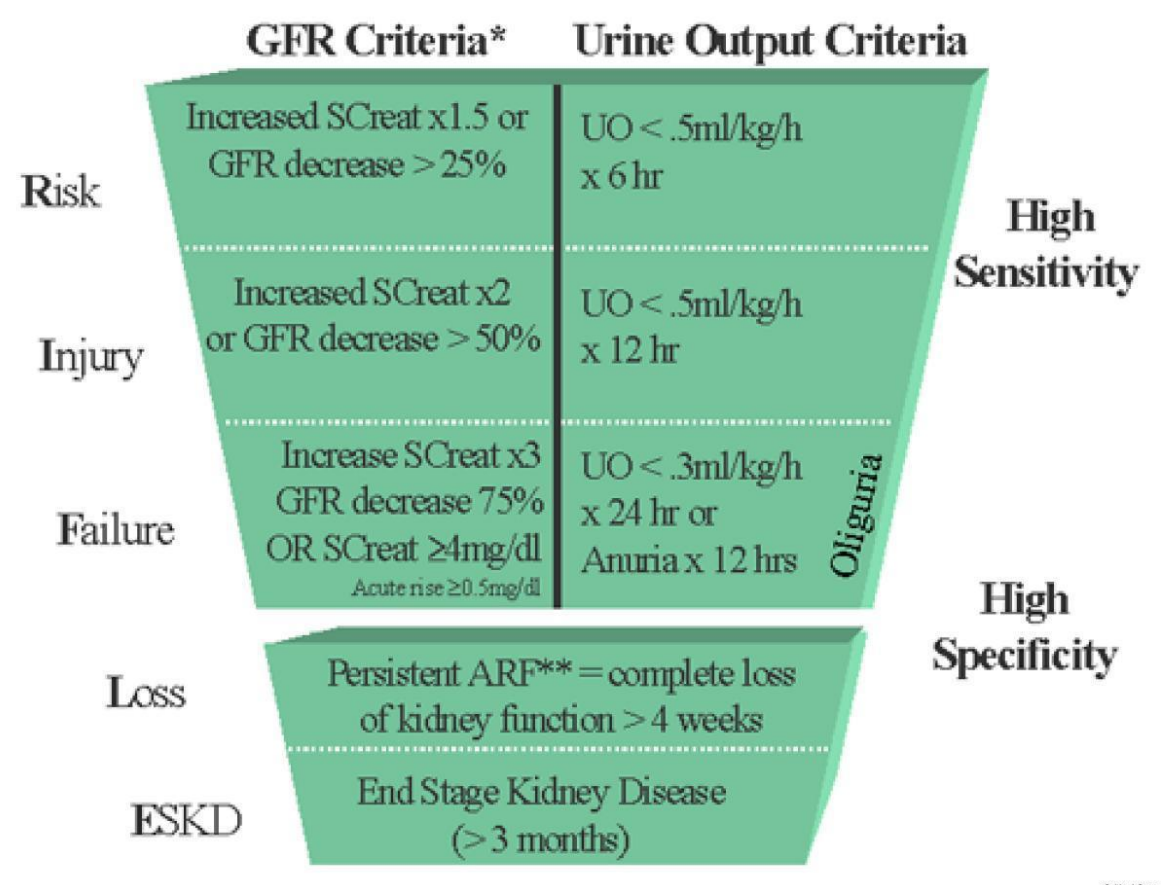

Abbildung 1.1 RIFLE-Kriterien nach Bellomo et al. 2004, S. R206. UO = UrinOutput (Diuresemenge), SCreat $=$ Serumkreatinin, GFR $=$ Glomeruläre Filtrationsrate, ARF $=$ Akutes Nierenversagen. 
RIFLE wurde zunächst zur am weitest verbreiteten Definition des akuten Nierenversagens, sowohl in der intensivmedizinischen als auch in der nephrologischen Literatur. Die Gesamtzahl der Patienten, die in Studien zur Validierung der RIFLE-Kriterien eingeschlossen wurden, geht über 200.000 hinaus (Kellum 2008).

Die Erkenntnis, dass eine Schädigung der Nieren schon vor deren völligem Versagen diagnostisch und therapeutisch relevant ist und bereits geringe Kreatininwertanstiege mit schlechterem Outcome, verlängerter Hospitalisierungszeit und erhöhter Mortalität assoziiert sind, ließ drei Jahre später durch die größere, interdisziplinäre, internationale Gruppe AKIN (Acute Kidney Injury Network) eine modifizierte Klassifikation entstehen. In diesem Zusammenhang wurde auch der früher geläufige Begriff des akuten Nierenversagens durch Acute Kidney Injury beziehungsweise akute Nierenschädigung abgelöst (Mehta et al. 2007).

Anhand der AKIN-Kriterien lässt sich die akute Nierenschädigung ausschließlich mit Hilfe der Diuresemenge und/oder des Serumkreatinins diagnostizieren und stratifizieren. Diese Modifikationen können wie folgt zusammengefasst werden: Zunächst legte AKIN eine numerische Stadieneinteilung 1, 2 und 3 statt R, I und F fest. Das RIFLE-Kriterium „Risk“ wurde durch das AKIN Stadium 1 erweitert, das bereits die Patienten mit einbezieht, die nur einen absoluten Serumkreatininanstieg von $0,3 \mathrm{mg} / \mathrm{dl}$ oder einen relativen 50 \% igen Anstieg vorweisen. Es wurde ein 48-Stunden-Zeitfenster für die erste Dokumentation eines der Kriterien eingerichtet. Patienten werden als Stadium 3 eingestuft, wenn ein Nierenersatzverfahren angewendet wird, unabhängig vom Kreatininwert oder der Diuresemenge (Mehta et al. 2007). Die unterschiedlichen Outcomes beziehungsweise Verlaufsformen der RIFLE-Klassifikation „Loss“ und „End Stage Renal Disease“ wurden in den AKIN-Kriterien nicht mehr berücksichtigt. 
Tabelle 1.1 AKIN-Klassifikationssystem der akuten Nierenschädigung modifiziert nach Mehta et al. 2007, S. R31.

\begin{tabular}{|c|c|c|}
\hline Stadium & Serumkreatinin-Kriterium & Diuresemenge-Kriterium \\
\hline 1 & $\begin{array}{l}\text { Serumkreatininanstieg } \geq 0,3 \mathrm{mg} / \mathrm{dl} \text { oder ein Anstieg } \\
\text { um } \geq 150 \% \text { bis } 200 \% \text { (1.5 - bis } 2 \text {-fach) des Aus- } \\
\text { gangswertes }\end{array}$ & $\begin{array}{l}\text { Weniger als } 0,5 \mathrm{ml} / \mathrm{kg} \text { pro } \\
\text { Stunde für mehr als } 6 \text { Stunden }\end{array}$ \\
\hline 2 & $\begin{array}{l}\text { Serumkreatininanstieg um mehr als } 200 \% \text { bis } \\
300 \% \text { (> 2- bis 3-fach) des Ausgangswertes }\end{array}$ & $\begin{array}{l}\text { Weniger als } 0,5 \mathrm{ml} / \mathrm{kg} \text { pro } \\
\text { Stunde für mehr als } 12 \text { Stun- } \\
\text { den }\end{array}$ \\
\hline 3 & $\begin{array}{l}\text { Serumkreatininanstieg um mehr als } 300 \% \text { (> 3- } \\
\text { fach) vom Ausgangswert oder Serumkreatinin } \\
\geq 4,0 \mathrm{mg} / \mathrm{dl} \text { mit einem akuten Anstieg von mindes- } \\
\text { tens } 0,5 \mathrm{mg} / \mathrm{dl} \text { oder Anwendung eines Nierener- } \\
\text { satzverfahrens }\end{array}$ & $\begin{array}{l}\text { Weniger als } 0,3 \mathrm{ml} / \mathrm{kg} \text { pro } \\
\text { Stunde für } 24 \text { Stunden oder } \\
\text { Anurie für } 12 \text { Stunden }\end{array}$ \\
\hline
\end{tabular}

\subsection{Anatomie und Physiologie der Niere}

Im Folgenden werden die Anatomie und die physiologischen Mechanismen der Nierendurchblutung erläutert, deren Kenntnis Voraussetzung zum Verständnis der Entstehung des prärenalen Nierenversagens ist, das auf ischämische Ursachen zurückzuführen ist.

\subsubsection{Anatomie}

Die Niere ist ein paarig angelegtes Organ, das sich im Retroperitonealraum befindet. Die Nieren setzen sich zusammen aus dem Nierenparenchym, der Nierenrinde und mark, den Markpyramiden, dem Nierenbecken und den Nierenkelchen. Jede Niere besteht aus circa einer Million Nephronen, die die funktionelle Einheit bilden. Das Nephron besteht aus einem Glomerulus mit Bowmannscher Kapsel und Kapillärknäuel. In der glomerulären Kapillare wird der Primärharn in den Bowmannschen Kapselraum filtriert. Die Bowmannsche Kapsel setzt sich in das Tubulussystem fort, dem proximalen Tubulus, Henle-Schleife, distalem Tubulus und Sammelrohr. Dann folgen die Kelche des Nierenbe- 
ckens. Bei einer Reduktion der Anzahl funktionierender Nephrone um mehr als 60-70\% kommt es zur Niereninsuffizienz (Krautzig 2004).

\subsubsection{Physiologie der Nieren}

\subsubsection{Nierendurchblutung und glomeruläre Filtrationsrate}

Die Nierendurchblutung nimmt etwa $25 \%$ des Herzzeitvolumens ein. Circa 1,2 Liter fließen pro Minute durch die Nieren mit ihrer besonderen Gefäßarchitektur: Die Vasa afferentia versorgen die glomerulären Kapillarschlingen und die Vasa efferentia versorgen über die Vasa recta die peritubulären Kapillaren des Kortex (Krautzig 2004).

Die hohe renale Durchblutung dient der Filtratbildung und damit der Regulationsund Ausscheidungsfunktion der Nieren. 90 \% des renalen Blutflusses erhält die Nierenrinde, der Ort der Filtratbildung (Silbernagl 2005). Durch Autoregulation über die Widerstandsgefäße in den Vasa afferentia wird die Nierendurchblutung weitgehend konstant gehalten, sodass systolische Blutdruckschwankungen zwischen 80 und $170 \mathrm{mmHg}$ zu keiner wesentlichen Änderung der Nierendurchblutung führen (Silbernagl 2005). Bei Erhöhung des transmuralen Druckes und bei Dehnung der glatten Muskelzellen reagieren die präglomerulären Nierengefäße, die Interlobulararterien und die afferenten Arteriolen mit einer Kontraktion. Diese aktive myogene Reaktion auf transmurale Druckdifferenzen bezeichnet man als Bayliss-Effekt. Dies ermöglicht eine von der treibenden Druckdifferenz unabhängige lokale Durchblutung: die Autoregulation der Nierendurchblutung. Dies wiederum ermöglicht eine konstante GFR. Erst bei systolischen Blutdruckwerten jenseits von circa $170 \mathrm{mmHg}$ oder $80 \mathrm{mmHg}$ ist die Autoregulationsgrenze erreicht, sodass die GFR direkt proportional zum arteriellen Blutdruck zu- beziehungsweise abnimmt (Silbernagl 2005).

Die glomeruläre Filtrationsrate wird durch den effektiven Filtrationsdruck, der durch den hydrostatischen Druck abzüglich des onkotischen Drucks im Plasma entsteht, beeinflusst. In einer Schocksituation oder bei Nierenarterienstenose mit Druckabfall über der Stenose kommt es zu Störungen des Filtrationsdrucks. Weiterhin ist die GFR abhängig von der Intaktheit der glomerulären Basalmembran und von der Filtrationsfläche, die beispielweise bei reduzierter Nephronanzahl bei chronischer Niereninsuffizienz verändert ist (Krautzig 2004). 


\subsubsection{Funktionen der Niere}

Die Funktionen der Niere umfassen neben der Ausscheidung harnpflichtiger Substanzen die Anpassung des Intravasal- und Extrazellulärvolumens über die Regulation des Wasser- und Elektrolythaushaltes und die Säure-Basen-Homöostase. Darüber hinaus zeigt die Niere endokrine und parakrine Wirkungen, spielt eine Rolle in der Blutdruckregulation, der Blutbildung und im Knochenstoffwechsel.

Harnpflichtige Substanzen wie Kreatinin, Ammoniak, Harnstoff, Harnsäure und Pharmaka werden passiv durch glomeruläre Filtration oder aktiv durch tubuläre Sekretion ausgeschieden. Andere Substanzen wie Glukose, Aminosäuren und Peptide können über energieabhängige aktive Transportvorgänge rückresorbiert werden.

Täglich werden circa 170 Liter Primärharn durch die glomeruläre Kapillarmembran in die Bowmannsche Kapsel und das Tubulussystem abfiltriert, man spricht von der glomerulären Filtrationsrate in Filtrationsvolumen pro Minute. Circa 168 der 170 filtrierten Liter werden rückresorbiert, zu größten Anteilen im proximalen Tubulus (65 \%), ferner im distalen Tubulus und im Sammelrohr. Die Rückresorption im Sammelrohr wird durch das antidiuretische Hormon, das an membranständige V2-Rezeptoren im Sammelrohr bindet und über cAMP den Einbau von Aquaporinen in die Zellmembran bewirkt, reguliert. Die Konzentrierung des Harns erfolgt mittels eines aktiven kortikomedullären osmotischen Druckgradienten, der nach dem Gegenstromprinzip zwischen dem absteigenden und aufsteigenden Schenkel der Henleschleife erzeugt wird.

Die Regulation des Säure-Basen-Haushaltes erfolgt über folgende Mechanismen: Bicarbonat-Rückresorption im proximalen Tubulus, Ausscheidung von Säure-Äquivalenten als Ammoniumionen $\left(\mathrm{NH}_{4}^{+}\right)$und Sekretion freier Wasserstoffionen $\left(\mathrm{H}^{+}\right)$. Weiterhin findet eine Umwandlung von Wasserstoff und Dinatriumhydrogenphosphat $\left(\mathrm{Na}_{2} \mathrm{HPO}_{4}\right)$ zu Natriumdihydrogenphosphat $\left(\mathrm{NaH}_{2} \mathrm{PO}_{4}\right)$ und Natrium $\left(\mathrm{N}^{+}\right)$statt, das als titrierbare Säure, Harnsäure oder Zitronensäure ausgeschieden wird (Krautzig 2004).

Mit dem Renin-Angiotensin-Aldosteron-System nimmt die Niere eine zentrale Rolle in der Blutdruckregulation ein. In den Granulosazellen des juxtaglomerulären Apparates wird Renin gebildet, das als proteolytisches Enzym die Bildung von Angiotensin II reguliert. Sinkt der Druck in den Nierenarterien um mehr als 10-15 mmHg, kommt es zur Freisetzung von Renin in den systemischen Kreislauf. Das Dekapeptid Angiotensin I wird vom 
aus der Leber stammenden Angiotensinogen abgespalten und mittels Hydrolyse durch das Angiotensin-Converting-Enzym entsteht das aktive Oktapeptid Angiotensin II. Angiotensin II bewirkt eine Blutdrucksteigerung, einerseits durch Erhöhung des peripheren Widerstands durch direkte Vasokonstriktion und andererseits über die Freisetzung von Aldosteron in der Nebennierenrinde, welches Natrium retiniert und damit das Extrazellulärvolumen steigert.

In den Mitochondrien des proximalen Tubulus wird der wirksame Metabolit des Vitamin D, das Calcitriol, aus 25-OH-Cholecalciferol hydroxyliert, welcher den KalziumHaushalt reguliert.

Daneben wird in peritubulären Fibroblasten der Nebennierenrinde Erythropoetin (EPO) gebildet. Ein verminderter Sauerstoffpartialdruck, bei arterieller Hypoxie oder Anämie, stimuliert dessen Bildung. EPO erhöht im Knochenmark die Proliferation und Differenzierung der Erythrozytenvorstufen. Bei einer GFR unter circa $25 \mathrm{ml} / \mathrm{min}$ fällt die EPO-Produktion ab, was in einer renalen Anämie resultiert. Außerdem ist die Niere ein wichtiges Organ im Energiestoffwechsel, da neben der Leber auch sie Gluconeogenese betreibt (Silbernagl 2005). 


\subsection{Einteilung, Ätiologie und Pathophysiologie des ANV}

Das akute Nierenversagen kann anhand des Ortes der zugrunde liegenden schädigenden Ursache in prä-, intra- und postrenal eingeteilt werden.

Das akute Nierenversagen kann sich entweder bei vorheriger normaler Nierenfunktion (acute) oder auch bei bereits vorbestehender eingeschränkter Nierenfunktion entwickeln (acute on chronic renal failure).

Weiterhin kann man eine Einteilung durch Quantifizierung der Diuresemenge vornehmen. Man unterscheidet normurische, polyurische, oligurische und anurische Verläufe. Eine Normurie liegt im Bereich von $1-1,5 \mathrm{l} / 24 \mathrm{~h}$ oder 0,5 - $1 \mathrm{ml} / \mathrm{min}$ vor. Die Polyurie fordert eine Urinausscheidung von mehr als $2800 \mathrm{ml} / 24 \mathrm{~h}$. Oligurie ist definiert durch weniger als $500 \mathrm{ml}$ Urinausscheidung in 24 Stunden, eine Anurie liegt bei einer Ausscheidung von weniger als $100 \mathrm{ml}$ Urin in 24 Stunden vor. Bis zu 30 \% der Fälle verlaufen jedoch normo- oder polyurisch, nur mit einem Anstieg des Serumkreatinins als Leitsymptom (von Baeyer 2011).

Außerdem lässt sich das AKI entsprechend der Höhe der Retentionsparameter klassifizieren. Hierzu siehe Punkt 1.1.2 RIFLE und AKIN.

\subsubsection{Klassische Einteilung: Prä-, intra- und postrenal}

Klassischerweise unterscheidet man pathophysiologisch zwischen dem prärenalen, renalen und postrenalen akuten Nierenversagen.

Die Übergänge sind fließend zu betrachten, da ein prä- oder postrenales Nierenversagen über die eintretende Tubulusschädigung häufig auch ein intrarenales Nierenversagen bedingt. 


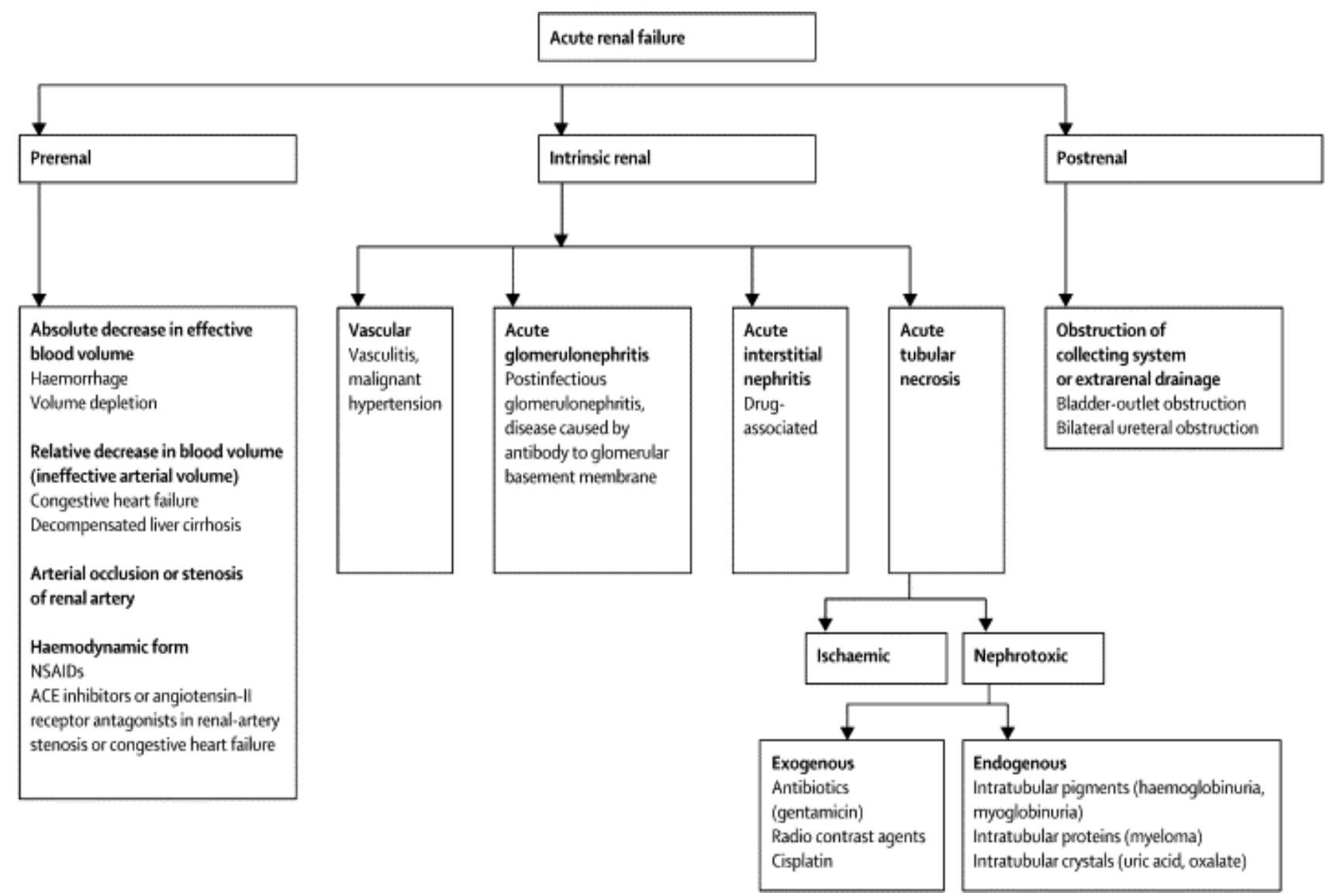

Abbildung 1.2 Ätiologische Einteilung mit den wichtigsten Ursachen des akuten Nierenversagens nach Lameire et al. 2005, S. 418. NSAIDs = Nicht Steroidale Antirheumatika, $\mathrm{ACE}=$ Angiotensin-Converting-Enzym.

\subsubsection{Prärenales Nierenversagen}

Sinkt der arterielle Blutdruck stark ab, so verringern sich die Nierendurchblutung und die GFR. Im Schockzustand kommt es zur peripheren Vasokonstriktion, wodurch die Nierendurchblutung stark abnimmt. Dies resultiert in einer Abnahme bis hin zum Versagen der Nierenfunktion, man spricht vom prärenalen akuten Nierenversagen. Nach Normalisierung des Blutdrucks kommt es auch zur Normalisierung der Nierenfunktion.

Das prärenale Nierenversagen stellt mit circa $75 \%$ der Fälle den größten Anteil. Die meisten Fälle sind zirkulatorisch-ischämisch bedingt. Dies tritt vor allem postoperativ oder posttraumatisch auf. Jede Störung, die zu Hypovolämie und Abnahme des effektiven Plasmavolumens führt, kann die Nierenperfusion beeinflussen und ein prärenales Nierenversagen induzieren, beispielsweise Hämorrhagien, gastrointestinaler Volumenverlust, Exsikkose, Pankreatitis, Leberinsuffizienz oder nephrotisches Syndrom mit Hypalbuminämie. Auch durch akute kardiale Dekompensation zum Beispiel nach Lungenembolie, Myo- 
kardinfarkt, Herzrhythmusstörungen, Perikardtamponade und weiteren Ursachen kommt es zu einer akuten Kreislaufinsuffizienz, Zentralisierung des Blutflusses, peripherer Vasokonstriktion und zur renalen Ischämie. Schockzustände jeglicher Ursache können zu renaler Minderperfusion führen, die in einem akuten Nierenversagen resultieren kann. Weiterhin werden Niereninfarkte bei Nierenarterienstenosen, Embolien oder hyaline Thromben, die zu Stenosen in den Arteriolen oder Glomeruli und zu renovaskulärer Minderperfusion führen, dem prärenalen Nierenversagen untergeordnet. Als pharmakologisch induziertes prärenales ANV sei eine ACE-Hemmer-Therapie in Kombination mit Antiphlogistika, durch Hemmung der Prostaglandinsynthese, Thrombozytenaggregationshemmer oder Diuretika insbesondere bei vorbestehender Nierenarterienstenose erwähnt. Prostaglandine bewirken eine Vasodilatation des Vas afferens und Angiotensin II eine Vasokonstriktion des Vas efferens mit Zunahme des Filtrationsdrucks. Wird die Synthese von Prostaglandinen beziehungsweise Angiotensin II gehemmt, ist die Autoregulationsfähigkeit der Nieren gestört und es kommt zur Abnahme der glomerulären Filtrationsrate.

\subsection{Pathophysiologie des zirkulatorisch-ischämisch-bedingten prärenalen Nierenversagens}

Die Ischämie führt zu einem Sauerstoff- und Substratmangel sowie einer Akkumulation von Stoffwechselprodukten. Bereits nach fünf Minuten liegt ein ATP-Mangel vor, sodass die $\mathrm{Na}+/ \mathrm{K}+/$ ATPase nicht ausreichend aufrecht erhalten werden kann, mit der Folge, dass die Resorptions- und Sekretionsleistungen der Tubuli abnehmen (Silbernagl 2005). Die Tubuluszellen quellen durch Wassereinstrom auf, sodass die Barrierefunktion der Zellmembran zusammenbricht. Es folgt ein Anstieg der intrazellulären Kalziumkonzentration, der zu einer Störung der Homöostase der Zellen führt und in einer weiteren Zellschwellung resultiert (Riede et al. 2004). Die Zellschwellung bedingt eine weitere Reduktion der Durchblutung und des Harnabflusses durch Verengung von sowohl Blutgefäßen als auch Tubuluslumina (Silbernagl 2005). „Werden nur einzelne Zellen geschädigt, so lösen sie sich von der Basalmembran ab, und benachbarte, noch erholungsfähige Zellen schließen die Epithellücken“ (Silbernagl 2005, S. 373). Durch Anstieg der Kalziumkonzentration werden außerdem vasoaktive Substanzen freigesetzt und intrazelluläre Enzyme aktiviert, die das Zytoskelett angreifen, was zur Abstoßung des Bürstensaums führt (Silbernagl 2005). Die primäre Schädigung des Tubulussystems führt dazu, dass die Natriumrückresorption vermindert wird, was über den tubuloglomerulären Rückkopplungsme- 
chanismus zusätzlich eine sekundäre glomeruläre Funktionseinschränkung nach sich zieht (Alpers und Fogo 2007).

\subsubsection{Intrarenales Nierenversagen}

Dem intrarenalen Nierenversagen liegt eine strukturelle Schädigung des Nierenparenchyms zu Grunde. Häufig kommt es durch eine toxische Tubulusschädigung zur akuten Nierenfunktionsstörung: entweder exogen in Folge von Medikamenten-Einnahmen (vor allem Antibiotika der Aminoglykosidgruppe) und Kontrastmittelgabe und bakteriellen Endotoxinen, oder auf endogenem Wege durch Rhabdomyolyse und Hämolyse und Hyperkalziämie. Weiterhin können interstitielle Erkrankungen - wie die akute interstitielle Nephritis - und glomeruläre Erkrankungen, insbesondere die Glomerulonephritiden vom rapid-progressiven und postinfektiösen Typ, ein intrarenales akutes Nierenversagen induzieren. Als seltenere Ursachen sind thrombotische Mikroangiopathien zu nennen, wie DIC (disseminierte intravasale Gerinnung), HUS (hämolytisch urämisches Syndrom) und TTP (thrombotisch thrombozytopenische Purpura).

Außerdem kann jedes länger bestehende prärenale Nierenversagen sekundär in ein intrarenales übergehen, da die Nephrone und Endothelien der Nierengefäße durch die prärenal verursachte Ischämie geschädigt werden (Silbernagl 2005). Man spricht dann von einer ischämisch induzierten akuten Tubulusnekrose, die häufigste Ursache des akuten Nierenversagens. Morphologisch ist die akute Tubulusnekrose charakterisiert durch nekrotische Veränderungen im proximalen Tubulus, Proteinaustritt im distalen Tubulus und ein interstitielles Ödem (Alpers und Fogo 2007).

\subsubsection{Postrenales Nierenversagen}

Das postrenale Nierenversagen entsteht durch Obstruktion der Harnwege mit Rückstau in eine oder beide Nieren, zum Beispiel durch eine Nephro- oder Urolithiasis, eine retroperitoneale Fibrose oder ein retroperitoneales Hämatom, eine mechanische Blasenentleerungsstörung durch eine Prostatahyperplasie, Harnröhrenstrikturen oder obstruktionen, oder neurogene Blasenentleerungsstörung. Auch können Tumore der 
Harnwege und Harnblase oder andere komprimierende Neoplasien im Bauchraum und angeborene Fehlbildungen zur akuten Niereninsuffizienz führen (Krautzig 2004).

\subsubsection{Häufigkeiten der unterschiedlichen Formen}

Das prärenale Nierenversagen stellt den größten Anteil in 60 \% - 75 \% der Fälle, gefolgt vom intrarenalen Nierenversagen mit $20-35 \%$. Das postrenale Nierenversagen tritt am seltensten auf (5 \%) (Lameire et al. 2005; von Baeyer 2011).

Die akute Tubulusnekrose nach chirurgischen Eingriffen macht etwa 20 - $25 \%$ aller im Krankenhaus erworbenen akuten Nierenversagen aus. Ein großer Anteil darunter hat prärenale Ursachen (Lameire et al. 2005).

Fünf Prozent aller hospitalisierten Patienten entwickeln ein akutes Nierenversagen (Hou et al. 1983). 5 - $20 \%$ der kritisch kranken Patienten erleiden im Verlauf ihrer Erkrankung eine Episode der Nierenfunktionseinschränkung, oft auch im Rahmen eines Multiorganversagens (de-Mendona et al. 2000; Lameire et al. 2005). Akutes Nierenversagen tritt bei $19 \%$ der Patienten mit moderater Sepsis auf, bei $23 \%$ mit schwerer Sepsis und bei $51 \%$ im septischen Schock (Schrier und Wang 2004). Nach operativen Eingriffen liegt die Inzidenz des ANV zwischen 0,8\% und bis zu $40 \%$, abhängig von der Art des Eingriffs. Die Mortalität liegt zwischen $17 \%$ und $60 \%$, wieder abhängig von der Art des Eingriffs. In $10-20 \%$ der Fälle geht das akute Nierenversagen in eine chronische Niereninsuffizienz über (Bourgeois et al. 2009).

\subsection{Verlauf und Klinik}

Das akute Nierenversagen ist gekennzeichnet durch einen plötzlichen Rückgang der inkretorischen und exkretorischen Funktionen der Niere. Die Klinik der akuten Nierenschädigung zeigt einen phasenartigen Verlauf. Es kommt zunächst zur akuten Einschränkung oder zum völligen Ausbleiben der Urinproduktion. Gleichzeitig steigt die Konzentration der harnpflichtigen Substanzen im Blut an und das spezifische Uringewicht ist wegen reduzierter tubulärer Konzentrationsleistung vermindert. Diese Phase wird als oligoanurisch bezeichnet. Nach Kreislauferholung folgt die polyurisch-hypostenurische Phase, in der die Urinproduktion wieder aufgenommen wird. Da die Konzentrations- und Rückre- 
sorptionsfähigkeiten der Tubuluszellen noch nicht vollständig wiederhergestellt sind, wird zunächst überschießend Harn produziert (Riede et al. 2004).

Die Anurie oder kritische Oligurie sind frühzeitige, wenn auch unspezifische Indikatoren der akuten Nierenschädigung. Diuresemengen-Veränderungen können lange vor laborchemischen Veränderungen auffällig werden. Das prärenale Nierenversagen zeigt sich fast immer mit einer Oligurie, obwohl auch nicht-oligurische Verläufe beschrieben wurden und eine Nierenschädigung trotz normaler Urinproduktion vorhanden sein kann. Das postrenale und renale Nierenversagen kann mit jeder Verlaufsform einhergehen von Anurie bis Polyurie (Lameire et al. 2005).

\subsection{Postoperatives AKI nach Kardiochirurgie}

Die postoperative akute Nierenfunktionsstörung nach herzchirurgischen Eingriffen unter Verwendung der Herz-Lungen-Maschine (HLM) ist eine häufige und gravierende Komplikation (Conlon et al. 1999; Haase et al. 2007; Mangano et al. 1998; Parolari et al. 2012; Thakar et al. 2005). Die pathophysiologischen Mechanismen der Herz-LungenMaschine-abhängigen Nierenschädigung werden im Kapitel 1.5.3 (S. 16) ausführlich erläutert.

\subsubsection{Verwendung der Herz-Lungen-Maschine}

Zahlreiche Operationen an den großen Gefäßen und am Herzen selbst sind nur am nicht schlagenden Herzen möglich. Hierzu müssen Herz und Lungen stillgelegt und aus dem normalen Kreislauf ausgeschaltet werden. Eine externe Herz-Lungen-Maschine übernimmt ihre Funktion, ein Vorgang, der als extrakorporale Zirkulation bezeichnet wird. Sie kann über mehrere Stunden aufrechterhalten werden und wird im Rahmen der Herzchirurgie bei folgenden Operationen durchgeführt: Aortokoronarer Bypass (ACB), Herzklappenersatz, Rekonstruktion von Herzklappen und Eingriffe an der Aorta ascendens, sowie für die Korrektur kongenitaler Herzfehler (Larsen 2010), die im Rahmen dieser Studie jedoch nicht mit eingeschlossen wurden. 


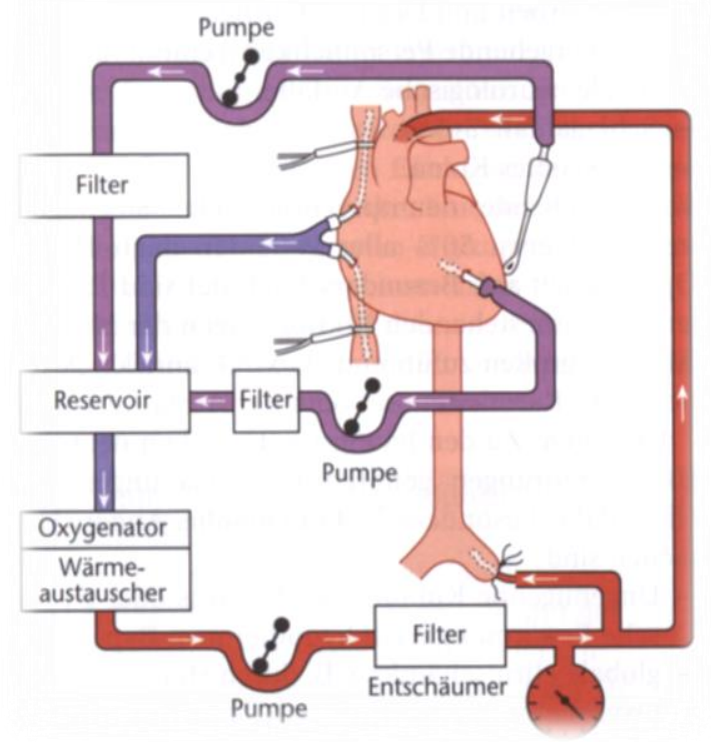

\section{Abbildung 1.3 Schema der extrakorporalen Zirkulation}

nach Larsen 2010, S. 1258.

Während der extrakorporalen Zirkulation fließt das gesamte venöse Blut über Kanülen und Schläuche aus beiden Hohlvenen in die Herz-Lungen-Maschine. Von hier wird es nach Oxygenierung und Eliminierung von $\mathrm{CO}_{2}$ über die Aorta oder die Femoralarterie in den arteriellen Kreislauf des Patienten zurückgepumpt. In Deutschland werden jährlich circa 100.000 Operationen unter Verwendung der Herz-Lungen-Maschine durchgeführt, weltweit über eine Million (Larsen 2010).

\subsubsection{Inzidenz und Auswirkungen auf Mortalität}

Mit einer Gesamtinzidenz von $1 \%$ - $30 \%$ treten akute Nierenschädigungen sehr häufig auf (Dasta et al. 2008). In der Literatur finden sich weit streuende Angaben zur Inzidenz, was einerseits auf eine bis zur Einführung der RIFLE- und AKIN-Klassifikationen lange fehlende einheitliche Definition des Nierenversagens zurückzuführen ist, andererseits auf zentrenabhängige Faktoren, verschiedene Operationsverfahren und -techniken (Candela-Toha et al. 2008; Falvo et al. 2008).

AKI mit Dialysepflicht nach kardiochirurgischen Eingriffen mit HLM-Einsatz tritt in bis zu 5 \% der Fälle auf, weitere 8-15\% verzeichnen eine moderate Erhöhung des Serumkreatininwertes von $1,0 \mathrm{mg} / \mathrm{dl}$, einen leichten Anstieg des Kreatininwertes gegenüber 
dem präoperativen Ausgangswert von mindestens $25 \%$ finden sich bei $50 \%$ der Patienten (Haase et al. 2007).

Die Wahrscheinlichkeit, ein postoperatives AKI zu erleiden, ist abhängig von verschiedenen Faktoren. Patienten mit besonders hohem Risiko eines HLM-assoziierten AKI können bereits anhand präoperativer Risikofaktoren identifiziert werden: Patienten weiblichen Geschlechts, mit präoperativem Kreatininwert über 1,2 mg/dl, insbesondere über 2,1 mg/dl, präoperativer IABP, mit reduzierter linksventrikulärer Funktion, einer COPD (Thakar et al. 2005) oder einem insulinabhängigen Diabetes mellitus (Parolari et al. 2012; Thakar et al. 2005). Daneben ist das Risiko eines AKI abhängig von intraoperativen Variablen wie der Art des Eingriffes, Elektiv- oder Notfalleingriff (Thakar et al. 2005), der Transfusion von Blutkomponenten (Haase und Shaw 2010) - insbesondere Erythrozytenkonzentrate (Parolari et al. 2012)-, HLM-Zeit und Aortenklemmzeit, sowie von postoperativen Risikofaktoren wie eine Re-Thorakotomie (Thakar et al. 2005) oder dem Einsatz von Inotropika und Antiarrhythmika (Parolari et al. 2012).

AKI ist nicht nur eine ernstzunehmende postoperative Komplikation, sondern bringt auch einen signifikant steigenden Kostenaufwand mit sich, insbesondere wenn der Einsatz eines Nierenersatzverfahrens notwendig wird. Schlechtes Outcome führt zu verlängerten Krankenhaus- und Intensivstationaufenthalten, doppelt so lange bei AKI und mehr als fünfmal so lange bei Dialyseeinsatz, und zu einem größeren Bedarf an spezialisierten Langzeit-Pflegeeinrichtungen (Mangano et al. 1998).

Die Gesamtmortalität nach Operationen mit HLM liegt zwischen 2 und $8 \%$. Bei Auftreten eines postoperativen akuten Nierenversagens hingegen steigt die Mortalitätsrate exponentiell an, alle Stadien des AKI sind mit einer erhöhten Mortalität assoziiert. Bei schweren AKI, die ein Nierenersatzverfahren benötigen, steigt die Mortalitätsrate auf $60 \%$ an (Bove et al. 2005; Thakar et al. 2005).

Chertow et al. berichten, dass ein Kreatininanstieg mit hohem Mortalitätsrisiko korreliert. Sie konnten zeigen, dass bereits geringe Veränderungen der Nierenfunktionen mit einem allgemein schlechteren Outcome assoziiert sind, sodass Nierenfunktionsstörungen entscheidend für eine schlechte Prognose von Patienten sind (Chertow et al. 2005). Genauso stellte die Arbeitsgruppe um Loef eine erhöhte Mortalität bei einer Serumkreatininerhöhung um nur $25 \%$ während der ersten postoperativen Woche fest (Loef et al. 
2005). Lassnigg et al. fanden eine doppelt so hohe Mortalitätsrate bei kardiochirurgischen Patienten mit nur geringen Serumkreatininanstiegen von $<0.5 \mathrm{mg} / \mathrm{dl}$ innerhalb von 48 Stunden postoperativ gegenüber Patienten mit einem Abfall des Serumkreatinins. Weiterhin stellten sie fest, dass die wiederholte Messung von Serumkreatininkonzentrationen während der ersten 48 Stunden und die Bestimmung des relativen Anstiegs die effektivste Methode zur Identifizierung von Patienten mit Risiko für ein schlechtes postoperatives Outcome nach kardialen Operationen ist. Sie sei besser als die alleinige Anwendung der RIFLE- oder AKIN-Kriterien, wobei RIFLE noch weniger Trennschärfe aufwies als AKIN (Lassnigg et al. 2008).

Da das postoperative akute Nierenversagen nicht nur den therapeutischen Aufwand und die damit verbundenen Kosten, sondern auch die Mortalität erheblich erhöht (Bahar et al. 2005; Mangano et al. 1998; Provenchere et al. 2003), muss eine effektive präventive Therapiestrategie etabliert werden. Zwar existieren evidenzbasierte Therapieansätze zur Optimierung der Nierenfunktion (Barr und Kolodner 2008; Bellomo 2005; Jones und Lee 2007; Kellum et al. 2005; Merten et al. 2004; Sisillo et al. 2008), die jedoch nicht unselektiert bei allen Patienten angewendet werden können. Die frühzeitige, idealerweise bereits präoperative Identifikation von Patienten mit erhöhtem Risiko einer akuten Nierenschädigung ist daher eine unabdingbare Voraussetzung für den gezielten Einsatz nephroprotektiver Maßnahmen.

\subsubsection{Ursachen für die hohe Inzidenz eines AKI nach kardiochirurgi- schen Operationen}

Es existieren verschiedene Mechanismen, die zu einer postoperativen Nierenschädigung nach kardiochirurgischen Operationen unter Einsatz von HLM führen. Es ist wichtig, die zentralen Mechanismen zu identifizieren, um eine entsprechende präventive Therapiestrategie entwickeln zu können.

Zu den zentralen Mechanismen des HLM-abhängigen AKI gehören die perioperative hämodynamische Instabilität, der beeinträchtigte renale Blutfluss mit renaler Hypoperfusion und der unphysiologische nicht-pulsatile Blutfluss sowie die ischämische Reperfusion. Der HLM-Einsatz führt zur Aktivierung von Entzündungskaskaden, die zu einem postoperativen SIRS (systemic inflammatory response syndrome) führen können und außerdem zu 
oxidoinflammatorischem Stress durch die Bildung von freien Radikalen. Andere Ursachen, die zur postoperativen Nierenschädigung beitragen, sind Mikroembolisation in den Nierenarterien, Hypothermie, Hämoglobinämie, Myoglobinämie und exogene Nephrotoxine wie nephrotoxische Antibiotika, NSAR und Anästhetika (Billings et al. 2011; Haase et al. 2007; Mack et al. 2004; Zanardo et al. 1994).

\subsubsection{Renale Hypoperfusionsschädigung}

Die Nieren gehören zu den am besten durchbluteten Organen des menschlichen Körpers, sodass sie besonders anfällig für eine ischämische Schädigung sind. Metabolische Beanspruchungen durch tubuläre Reabsorption bewirken die ischämische Vulnerabilität der renalen Perfusion mit niedrigen Sauerstoffpartialdrücken im Nierenmark von 10 $20 \mathrm{mmHg}$. Aus diesem Grund ist die Aufrechterhaltung des physiologischen Blutflusses durch einen ausreichenden Perfusionsdruck (MAP) vor, während und nach HLM-Einsatz essentiell zur Prävention des postoperativen AKI.

Die perioperative hämodynamische Instabilität, über die Grenzen der Autoregulationsfähigkeit des Nierenperfusionsdruckes hinaus, führt einerseits zu renaler Minderperfusion und Hypoxie im Nierenmark, die an sich eine Nierenschädigung auslösen können. Jegliche Diskrepanz zwischen Sauerstoffbedarf und Angebot kann sich äußerst kritisch auf die Nierenfunktion auswirken (Haase et al. 2007). Dies wird weiter verstärkt durch eine Anämie, hervorgerufen durch Hämodilution während und nach der extrakorporalen Zirkulation, die mit einer erheblich reduzierten Sauerstofftransportkapazität einhergehen kann (Ranucci et al. 2005). Zusätzlich beeinflusst die Hämodilution auch die Mikrozirkulation in der Niere, sodass die Hämodilution an sich als ein entscheidender Faktor im Hinblick auf die Entstehung der Nierenschädigung anzusehen ist (Karkouti et al. 2005). Andererseits kommt es durch die anämisch-hypoxischen Bedingungen auch zur vermehrten Bildung von freien Radikalen in den Mitochondrien, die in einer weiteren Schädigung der Niere resultieren. Es wird außerdem angenommen, dass nicht nur ischämische oder hypoxische intraoperative Bedingungen selbst zu einer Nierenschädigung führen, sondern auch deren Reperfusion (Haase et al. 2007). 


\subsubsection{Oxido-inflammatorischer Stress}

Oxidativer Stress ist eine wichtige Ursache der Nierenschädigung bei Patienten, die einer extrakorporalen Zirkulation ausgesetzt sind. Getriggert durch Oberflächenkontakte, Ischämie und Reperfusion kommt es zu einer Akute-Phase-Reaktion, die eine Reihe von postoperativen Komplikationen mit sich bringt. Eine Kaskade von Entzündungsreaktionen führt schlussendlich zur Aktivierung von Leukozyten und Endothelialzellen, die für Zelldysfunktionen in verschiedenen Organen verantwortlich ist (Paparella et al. 2002). Der Einsatz der HLM ist proinflammatorisch und aktiviert Komponenten des unspezifischen Immunsystems. Die Immunantwort beinhaltet die systemische, aber auch die in der Niere lokalisierte Bildung von Zytokinen wie TNF und IL-6, die die renale Mikrozirkulation beeinträchtigen und auf diesem Wege zur Tubulusschädigung führen (Haase et al. 2007).

Neben der Stimulation von neutrophilen Granulozyten und Entzündungsmediatoren kommt es zur Bildung von freien Radikalen. Vieles deutet darauf hin, dass die Bildung von freien Radikalen zu Tubuluszellnekrose führt und sie aufrecht erhält (Nath und Norby 2000). Zusätzlich berichteten Haase et al. von einer temporären intra- und postoperativen Einschränkung der antioxidativen Kapazitäten im Serum, was das Ausmaß der Schädigung durch freie Radikale noch weiter verstärkt (Haase et al. 2007).

\subsubsection{Hämolyse induziert Nierenschädigung}

Durch mechanische Beanspruchung der Erythrozyten durch die Komponenten der HML entstehen Scherkräfte und Schubspannung, die zur mechanischen Hämolyse führen. Hierzu tragen folgende Komponenten bei, deren hämolytische Wirkung durch hohe Flussgeschwindigkeit und hohe Druckzustände zusätzlich verstärkt wird:

Schläuche, Durchfluss-Querschnittsflächen, die Anzahl an Kreislaufverbindungsstücken, Rauheitsgrad der Oberflächen, Filter, Grenzflächen des Oxygenators und Blutaspiration während des Kardiotomie-Sogs (Skrabal et al. 2006). Das Ausmaß der Erythrozytenzerstörung durch die Herz-Lungen-Maschine korreliert mit der Dauer der extrakorporalen Zirkulation. Folglich findet umso mehr Hämolyse statt, je länger die extrakorporale Zirkulation andauert, und dementsprechend zirkuliert mehr freies Hämoglobin. Es konnte weiterhin gezeigt werden, dass die Dauer der extrakorporalen Zirkulation mit der Inzidenz des AKI korreliert. Demzufolge scheint die Hämolyse mit der Freisetzung von Hämoglobin 
einen entscheidenden Beitrag zur Entstehung des AKI nach kardiochirurgischen Eingriffen zu leisten, was aktuell von großer klinischer Wichtigkeit ist (Haase et al. 2007).

Kardiochirurgische Patienten, die unter Verwendung der HLM operiert wurden und postoperativ ein AKI entwickeln, weisen im Vergleich zu Kontrollgruppen eine höhere Hämoglobinämie auf. Wird eine Nierenschädigung durch Freisetzung großer Mengen an Hämoglobin oder Myoglobin und einer daraus resultierenden Hämoglobinurie beziehunsweise Myoglobinurie ausgelöst, spricht man von einer sogenannten Pigmentnephropathie. Viele ursächliche Faktoren sind an der Freisetzung von Hämoglobin und Myoglobin ins Serum beteiligt, dazu gehören vor allem die Hämolyse durch HLM, die Rhabdomyolyse, aber auch andere Ursachen wie Transfusionsreaktionen, Malariainfektionen, mechanische Lyse durch Gefäßprothesen, Verbrennungen, chemische Agenzien, sowie genetische Defekte, die zu Membraninstabilitäten der Erythrozyten prädisponieren (Haase et al. 2007).

Außerdem konnte eine Korrelation zwischen der Menge des freien Hämoglobins, dem oxidativen Stress und dem postoperativen Anstieg des Serumkreatinins nachgewiesen werden. Dies weist darauf hin, dass freies Hämoglobin zum oxidativen Stress während des HLM-Einsatzes beiträgt und darauffolgend zur Nierenschädigung führt. Die Mechanismen, die zur Bildung dieses intraoperativen oxidativen Stresses führen und zur Pathogenese des postoperativen AKI beitragen, sind multifaktoriell und bis heute nicht vollständig erklärt. Hämproteine schädigen die Nierenzellen durch vielseitige Mechanismen, darunter Vasokonstriktion, Zylinderbildung und Lipid-Peroxidation. Erhöhtes freies Hämoglobin und erhöhte Myoglobinwerte wurden bereits als unabhängige Risikofaktoren zur Entstehung des AKI identifiziert (Benedetto et al. 2010), was die Annahme unterstützt, dass Hämproteine eine entscheidende Rolle in der Pathogenese des postoperativen AKI spielen (Billings et al. 2011; Vermeulen Windsant et al. 2010).

Hämoglobin und Myoglobin besitzen eine ähnliche chemische Kernstruktur, das Hämprotein. Im Zentrum des Hämproteins befindet sich das $\mathrm{Fe}^{2^{+}}$. Beide Moleküle können freies Eisen abgeben, das als Nephrotoxin wirkt, sodass von einer gleichen Pathogenese des AKI durch Hämoglobinurie und Myoglobinurie ausgegangen wird. Durch die oben beschriebene HLM-abhängige mechanische Hämolyse steigt der Plasmaspiegel von freiem Hämoglobin, das sich mit Haptoglobin zu einem Komplex verbindet (Haase et al. 2007). Unter normalen Umständen existiert Hämoglobin nicht in freier Form im Serum; nur 
wenn die Menge des freien Hämoglobins die Bindungskapazität von Haptoglobin überschreitet, erscheint Hämoglobin im Urin, da es in freier Form glomerulär filtrierbar ist (Keene und Jandl 1965). Es kommt zur Bildung von Hämoglobinzylindern mit Tubulusobstruktion und Tubuluszellnekrose. Weiterhin wird freies Eisen freigesetzt, das an der Bildung von freien Radikalen beteiligt ist. In Tierstudien konnte gezeigt werden, dass die Gabe von freiem Hämoglobin zu einem akuten Nierenversagen führt. Es ist anzunehmen, dass die Umwandlung von Hämoglobin zu Methämoglobin ein wichtiger pathophysiologischer Schritt in der Entstehung der Pigmentnephropathie ist. Das saure Milieu im tubulären Urin erleichtert diese Umwandlung. Methämoglobin präzipitiert im distalen Tubulus zu Zylindern, die in jeder Pigmentnephropathie gefunden werden können. Dies führt zur Tubulusobstruktion und schließlich zum Versiegen der Filtration (Haase et al. 2007; Jaenike 1967; Paller 1988). Im sauren Milieu und durch die Tubulusobstruktion kommt es zur vermehrten endozytotischen Aufnahme von freiem Hämoglobin in die proximalen Tubuluszellen, was mit einer proximalen Tubuluszellnekrose assoziiert ist. Ischämie triggert die Zylinderbildung, diese verstärkt die tubuläre Obstruktion, was wiederum die Hämoglobinaufnahme in den proximalen Tubuluszellen erleichtert. Es konnte gezeigt werden, dass eine zusätzliche ischämisch/hypoxische Reperfusion nach Okklusion der Nierenarterien zu einer drastischen Verschlimmerung der Pigmentnephropathie mit Bildung von ausgedehnten Methämoglobinzylindern und Nekrose des proximalen Tubulus führt (Haase et al. 2007).

Dagegen hat die Gabe von Hämoglobin in Tiermodellen unter alkalischen Bedingungen, wie sie durch eine prophylaktische Gabe von Natriumbicarbonat erzeugt werden können, keine Nierenschädigung zur Folge, und eine Urinalkalisierung vermindert das Ausmaß des akuten Nierenversagens. Es kommt darunter zu signifikant verminderter Umwandlung von Hämoglobin zu Methämoglobin, verminderter Bildung von Methämoglobinzylindern und dadurch weniger Tubulusobstruktion. Eine proximale Tubuluszellnekrose scheint unter diesen Umständen auch äußerst selten aufzutreten (Zager und Gamelin 1989). 


\subsubsection{Kardio-renales Syndrom}

Das CRS ist definiert als kardio-renales Syndrom, also als Dysfunktion von Herz und Nieren, wobei eine akute oder chronische Dysfunktion eines Organs eine akute oder chronische Dysfunktion des anderen induzieren kann. Der CRS-Typ 1 bezieht sich auf eine abrupte Verschlechterung der kardialen Funktion, die zu einer akuten Nierenschädigung führt. Die klinische Gewichtung der einzelnen Mechanismen, die primär zum akuten Herzversagen geführt haben, variieren von Patient zu Patient und von Situation zu Situation. Im akuten Herzversagen mit beeinträchtigter linksventrikulärer Ejektionsfraktion scheint das AKI gravierender zu sein, als bei Patienten mit erhaltener linksventrikulärer Funktion. Die dann beeinträchtigte Nierenfunktion wird durchweg als unabhängiger Risikofaktor für die Ein-Jahres-Mortalität bei Patienten mit akutem Herzversagen bestimmt (Ronco et al. 2008).

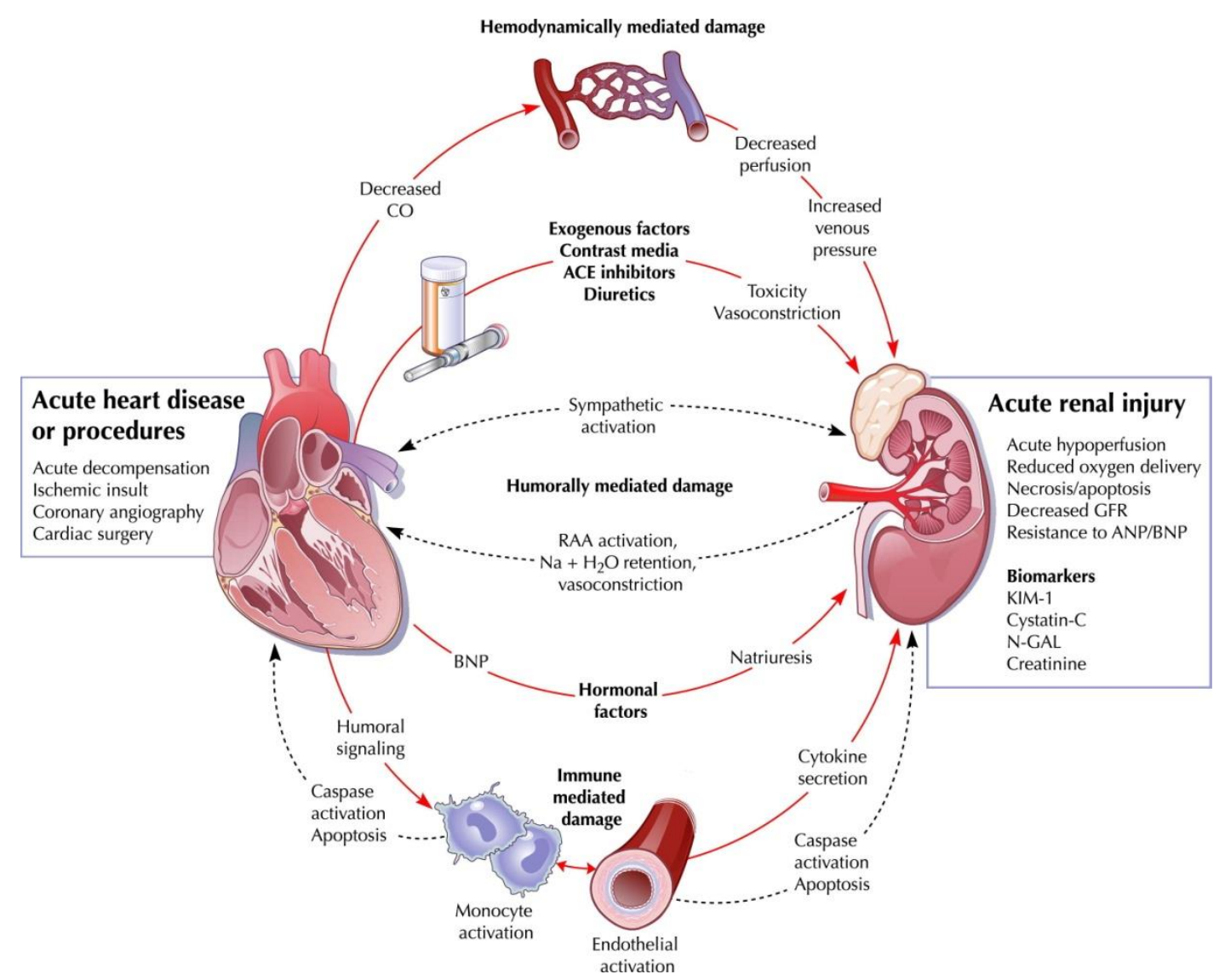

Abbildung 1.4 Kardiorenales Syndrom Typ 1 nach Ronco et al. 2008, S. 1529. ANP = Atriales-Natriuretisches Peptid, BNP = B-Typ-Natriuretisches Peptid, $\mathrm{CO}=$ Cardiac Output, $\mathrm{KIM}=$ Kidney-Injury-Molecule, $\mathrm{N}-\mathrm{GAL}=$ Neutrophilen-Gelatinase-assoziiertes Lipocalin, RAA = Renin-Angiotensin-Aldosteron-System. 


\subsection{Therapeutische Ansätze}

\subsubsection{Allgemeine Therapiekonzepte}

Bisher konnte für die Nierenschädigung selbst noch keine Therapie entwickelt werden, sodass der Fokus darauf liegt, zunächst die auslösenden Ursachen der Nierenschädigung frühzeitig zu detektieren, um diese effektiv zu therapieren.

Neben der kausalen Therapie der Grunderkrankung besteht eine weitere Strategie in der unterstützend orientierten symptomatischen Therapie. Hierzu zählt die Aufrechterhaltung aller oben beschriebenen Funktionen der Niere. Wichtig ist auch die Aufrechterhaltung und Optimierung des kardialen Outputs und des renalen Blutflusses. Zunächst ist die Überprüfung der gesamten Medikation mit gegebenenfalls Dosisanpassung der renal eliminierten Medikamente und Absetzen aller nephrotoxischen Komponenten obligatorisch. Weiterhin ist die Flüssigkeitszu- und ausfuhr zu bilanzieren und bei Bedarf eine Volumensubstitution durchzuführen. Auf einen ausgeglichenen Elektrolythaushalt und $\mathrm{pH}$-Wert, sowie die suffiziente Ausscheidung der harnpflichtigen Substanzen ist zu achten. Überschreiten die Parameter die tolerablen Grenzwerte, muss ein Nierenersatzverfahren eingeleitet werden. Weiterhin gilt es, Komplikationen wie beispielsweise Infektionen oder Lungenödeme zu vermeiden und gegebenenfalls zu therapieren. Frühe nutritive Unterstützung kann sinnvoll sein (Lameire et al. 2005).

Daneben stellt die präventive Therapie eine wichtige Säule der Behandlung akuter Nierenfunktionsstörung dar. Gerade hierzu ist die Abschätzung des individuellen Risikos der Entwicklung eines Nierenversagens wichtig. Nur dann können ausreichend nephroprotektive Maßnahmen zielgerichtet eingesetzt werden, wenn potentiell nephrotoxische Anwendungen durchgeführt werden müssen.

\subsubsection{Spezielle Therapiekonzepte}

\subsubsection{Therapie der Grunderkrankung}

Zu den häufigsten Ursachen des prärenalen akuten Nierenversagens zählen der septische Schock, der kardiogene Schock und die akute Hypovolämie. Ziel ist hierbei die Stabilisation der hämodynamischen Parameter sowie die strikte Vermeidung von Volu- 
mendefiziten. Die Aufrechterhaltung des Nierenperfusionsdruckes ist essentiell, wozu beispielsweise der Einsatz von Noradrenalin dienen kann (Leone und Martin 2008). Das Absetzen nephrotoxischer Substanzen stellt bei primär toxisch-intrarenalen Formen des AKI die kausale Therapie eines intrarenalen Nierenversagens dar. Zur Therapie des postrenalen Nierenversagens dient häufig die chirurgische Entfernung von Harnwegsobstruktionen, beispielsweise der Prostatahyperplasie.

\subsubsection{Sekundär präventive Maßnahmen}

Es existieren zahlreiche Behandlungsansätze zur pharmakologischen Prävention eines AKI. Die Arbeitsgruppe Nephrologie der European Society of Intensive Care Medivine (ESICM) publizierte 2010 ein Resumée derjenigen Maßnahmen, für die ein eindeutiger, evidenzbasierter präventiver Effekt gezeigt werden konnte (Joannidis et al. 2010). Dies beinhaltet insbesondere die Kreislaufstabilität mit spezieller Aufmerksamkeit gegenüber einem ausreichenden intravasalen Volumen (Bellomo 2005) durch adäquate Hydratation unter Vermeidung von hochmolekularem HES (Joannidis et al. 2010), Aufrechterhalten eines adäquaten mittleren arteriellen Blutdruckes von $60-65$ mmHg durch Einsatz von Vasopressoren im hypotensiven Schock mit Vasodilatation, weiterhin die Optimierung des Cardiac Outputs (Bellomo 2005) eventuell durch spezifische Vasodilatatoren unter strikter hämodynamischer Kontrolle zur Blutdrucksenkung; außerdem die kurzfristige Hämofiltration bei anhaltender Nierenfunktionsstörung nach Koronarintervention. Daneben sind alle nephrotoxischen Medikamente (insbesondere Amphotericin B und Aminoglykoside) abzusetzen (Joannidis et al. 2010).

\subsection{Volumentherapie}

Neben der kausalen Therapie eines ANV, hervorgerufen durch Hypovolämie, ist die Volumensubstitution auch zur Prävention einer Nierenfunktionsstörung wichtig. Es ist stets auf einen ausgeglichenen Volumenstatus zu achten (Bellomo 2005). Dabei sind der Einsatz von hochmolekularer Hydroxyethylstärke (10\% HES 250/0,5) oder Dextranen und unphysiologisch hohe Flüssigkeitsbelastungen unbedingt zu vermeiden, da sie pulmonale und kardiale Probleme zur Folge haben können. Bevorzugt eingesetzt werden sollten Gelantinelösungen, niedermolekulares HES und kristalloide Lösungen (Joannidis et al. 2010), hierunter Vollelektrolytlösungen, da isotonische Kochsalzlösungen zu einer hyperchlorä- 
mischen Azidose und darüber zu einer weiteren Verschlechterung der Nierenfunktion führen können (Wilcox 1983).

Die prophylaktische Volumenexpansion mit isotonischen Kristalloiden sollte bei Patienten mit hohem Risiko einer Kontrastmittel induzierten Nephropathie oder bei nicht vermeidbarem Gebrauch potentiell nephrotoxischer Medikamente durchgeführt werden (Joannidis et al. 2010).

\subsection{Dopamin}

Dreißig Jahre lang war das Konzept der low-dose-Dopamin-Therapie $(<5 \mu \mathrm{g} / \mathrm{kg} / \mathrm{min})$ bei oligurischen Patienten etabliert. Jedoch zeigte sich in großen multizentrischen Studien, dass Dopamin keinen protektiven Einfluss auf die Nierenfunktion hatte. Darüber hinaus konnten sogar negative Wirkungen auf die Splanchnikus-Oyxgenierung, Gastrointestinalfunktion, endokrinologische Funktionen und auf das Immunsystem nachgewiesen werden. Folglich besteht heute keine Indikation mehr für den Gebrauch von low-doseDopamin bei kritisch kranken Patienten (Friedrich et al. 2005; Holmes und Walley 2003; Joannidis et al. 2010).

\subsection{Diuretika}

Zur Prävention oder Verbesserung akuter Nierenfunktionsstörungen sind Schleifendiuretika nicht empfohlen. Es konnte keine Verbesserung des primären Outcomes, wie Erholung der Nierenfunktion und Mortalität nachgewiesen werden (Joannidis et al. 2010). Darüber hinaus wurde sogar beobachtet, dass die Gabe von Schleifendiuretika mit einer Verschlechterung der Nierenfunktion assoziiert sein kann, was darauf zurückzuführen ist, dass eine Diuretika-induzierte Hypovolämie zu Störungen der glomerulären Filtration führt. Des Weiteren nimmt der osmotische Druckgradient im Nierenmark unter dem Einfluss von Schleifendiuretika ab, wodurch es zu einer Abnahme der Markdurchblutung und damit der nutritiven Versorgung der Tubuluszellen kommt (Mason et al. 1981).

Dennoch können auch positive Effekte, die die Nierenfunktion verbessern können, verzeichnet werden: vermehrte Flussgeschwindigkeit mit Verhinderung von Tubuluslumenobstruktion durch Zelldetritus und Reduktion des Sauerstoffverbrauchs im Markraum (Bayati et al. 1990; Heyman et al. 1994). So gehört der Einsatz von Diuretika weiterhin zum klinischen Alltag, eine definitiv gerechtfertigte Indikation besteht allerdings nur bei kritischer Hypervolämie oder Hyperkaliämie (Bagshaw et al. 2007). 


\subsection{Vasopressoren}

Eine zielorientierte Therapie zur Aufrechterhaltung des arteriellen Mitteldrucks von $60-65 \mathrm{mmHg}$ ist essentiell. Hierzu sollten individuell angepasste Substanzen je nach Grunderkrankung zum Einsatz kommen. Im Fall einer vasoplegen Hypotension in Folge einer Sepsis oder eines SIRS sind Noradrenalin oder Dopamin als Vasopressoren der ersten Wahl zusammen mit Volumengabe einzusetzen (Joannidis et al. 2010). Zum Einsatz von prophylaktischer low-dose-Dopamingabe siehe oben.

\subsection{Vasodilatatoren}

Bei ausgeglichenem Volumenstatus und streng hämodynamisch überwachten Patienten, insbesondere in Hinsicht auf eine Hypotension, kann ein Vasodilatator zur protektiven Therapie eingesetzt werden. Bei der Wahl des geeigneten Vasodilatators müssen der Zustand des Patienten, die Begleitumstände und die geplanten Eingriffe sowie die Medikamentenverfügbarkeit beachtet werden. Bei kardiochirurgischen Patienten mit erhöhtem Risiko für ein AKI wird die prophylaktische Gabe von Fenoldopam, einem kurzwirksamen Partialagonisten am Dopamin-1-Rezeptor und Antihypertensivum, empfohlen. Weiterhin kann der Einsatz von natriuretischen Peptiden in Erwägung gezogen werden. Die Gabe von Theophyllin, einem nicht-selektiven Adenosin-A1- und -A2-RezeptorAntagonisten, konnte in verschiedenen experimentellen Studien eine Adenosin-A1Rezeptor-vermittelte afferente renale Vasokonstriktion und ein Kontrastmittelinduziertes Nierenversagen verhindern (Huber et al. 2006). Demzufolge kann es insbesondere bei akuten Interventionen mit Kontrastmittel empfohlen werden, wenn eine Hydratation nicht durchführbar ist (Joannidis et al. 2010).

\subsection{Hormonelle Therapie}

Bei chirurgischen Patienten ist eine Blutzuckerkontrolle zur Vermeidung von Hypound Hyperglykämien durchzuführen, was als Präventionsmaßnahme des AKI angesehen wird (Bellomo 2005; Bellomo et al. 2008). Eine altersentsprechende normale Blutzuckerkontrolle mit intravenöser Insulingabe nach etablierten Blutzuckerprotokollen hat sich als ausreichend herausgestellt, sodass eine intensivierte Insulintherapie nicht mehr empfohlen wird. Auch die routinemäßige Gabe von Thyroxin, Erythropoietin, Aktiviertem Protein C oder Glukokortikoiden zur Prävention eines AKI werden nicht empfohlen (Joannidis et al. 2010). 


\subsection{Metabolische Therapie}

Alle gefährdeten Patienten erhalten eine adäquate Ernährungsunterstützung, bevorzugt auf enteralem Wege, da hierdurch eine Wiederherstellung der Nierenfunktion erreicht werden kann (Joannidis et al. 2010). N-Acetylcystein zeigt widersprüchliche Effekte, sodass es bei besseren Alternativen nicht mehr zur Prophylaxe einer Nierenschädigung eingesetzt werden sollte (Bellomo et al. 2008), genauso wenig wie eine routinemäßige Gabe von Selen (Joannidis et al. 2010).

\subsection{Nierenersatztherapie}

Extrakorporale Nierenersatzverfahren gehören weniger zur Prävention, als vielmehr zur effektiven - allerdings auch aufwendigen - Behandlung eines kritischen AKI bei konservativ nicht mehr behandelbarer metabolischer Azidose, Hyperkaliämie, Urämie und Hypervolämie.

Es gibt die Möglichkeit einer periinterventionellen kontinuierlichen veno-venösen Hämofiltration (CVVH), beispielsweise zur Prävention einer Kontrastmittelnephropathie nach Herzkatheteruntersuchungen bei Hoch-Risiko-Patienten mit fortgeschrittener chronischer Niereninsuffizienz (Joannidis et al. 2010). Zur Durchführung eines Nierenersatzverfahrens muss individuell entschieden werden, ob die Therapie in einem frühen oder fortgeschrittenen Stadium der Nierenschädigung begonnen wird, welche tägliche Dialysedosis und welcher Therapiemodus (konvektiv oder diffusiv; kontinuierlich oder intermittierend) angewendet werden.

\subsubsection{Spezielle Therapiekonzepte in der Kardiochirurgie}

Die kausale Therapie einer postoperativen Nierenschädigung nach kardiochirurgischen Operationen erfordert zunächst die Kenntnis von deren Pathogenese, die im Kapitel 1.5.2 beschrieben wurde.

Da die perioperative hämodynamische Instabilität und der beeinträchtigte renale Blutfluss mit renaler Hypoperfusion zu den zentralen Mechanismen gehören, ist stets auf eine hämodynamische Stabilität des Patienten mit einem ausgeglichenen Volumenstatus, einem arteriellen Mitteldruck von mindestens 60-65 mmHg und einem adäquaten Cardiac Output zu achten. Hierzu dienen oben genannte Volumentherapiekonzepte und Vasop- 
ressoren oder als Maximaltherapievariante der Einsatz einer intraaortalen Ballonpumpe (IABP) (Bellomo et al. 2008).

Therapeutische Ziele sollten weiterhin die Reduzierung der HLM-induzierten Hämolyse und Hämoglobinämie sein. Dies könnte beispielsweise durch Einsatz von Off-pumpTechniken, Minimierung der Dauer der HLM-Verwendung (Bellomo et al. 2008), durch technischen Fortschritt der HLM-Zirkulation oder den Einsatz von Zentrifugalpumpen erreicht werden. Abgesehen von der Optimierung der technischen Durchführung, ist es notwendig, Strategien zur medikamentösen Prävention und Behandlung der Hämolyse zu entwickeln. Neben der Gabe von Natriumbicarbonat wird zurzeit der Einsatz von Haptoglobin diskutiert. Haptoglobin ist ein Transportprotein für freies Hämoglobin im Plasma, dessen Plasmakonzentration sofort nach Beginn der HLM unter die Nachweisgrenze sinkt. Dies weist darauf hin, dass auf Grund der Hämolyse die Bindungskapazität von Haptoglobin für Hämoglobin erschöpft ist und daraufhin Hämoglobin frei zirkulieren kann (Billings et al. 2011).

In verschiedenen Studien wurde die Aortenklemmzeit als unabhängiger Risikofaktor für die Entstehung einer akuten Nierenschädigung identifiziert (Parolari et al. 2012; Roh et al. 2012). Demzufolge ist neben der HLM-Zeit ebenfalls die Aortenklemmzeit möglichst kurz zu halten. Nach Wiedereröffnen der Aorta kommt es durch die ischämische Reperfusion der Aorta zu den bereits in Kapitel 1.5.2.2 beschriebenen Konsequenzen mit oxidativem Stress und inflammatorischen Reaktionen mit Apoptoseinduktion in den Nieren. Dies konnten Oyar et al. auch nach infrarenaler Abklemmung der Aorta zeigen (Oyar et al. 2012).

Das Ausmaß des oxidoinflammatorischen Stresses durch die Bildung von freien Radikalen und die Aktivierung von Entzündungskaskaden, die zu einem postoperativen SIRS führen können, sollte reduziert werden. Auch dies ist durch Optimierung der HerzLungen-Maschinen-Verwendung und der Aortenklemmzeit möglich.

Außerdem gibt es verschiedene Strategien bezüglich des intraoperativen Temperaturmanagements, die das Risiko eines postoperativen AKI beeinflussen (Coleman et al. 2011; Englberger et al. 2011; Mori et al. 2011; Roh et al. 2012). 


\subsubsection{Prävention des AKI durch Gabe von Natriumbicarbonat}

Ein saurer pH-Wert ist schädlich, indem die Bildung von Methämoglobin-Zylindern und die Bildung von tubulotoxischen freien Radikalen gefördert werden. In Anbetracht der Pathogenese des HLM-assoziierten AKI, die unter dem Punkt 1.5.3 (S. 16) beschrieben wurde, gibt es ausreichend pathophysiologische Argumente, den Urin-pH als wichtiges Behandlungsziel zu bestimmen, um die akute Nierenschädigung nach HLM-Einsatz zu verringern. Die Urinalkalisierung mit Natriumbicarbonat stellt einen vielversprechenden protektiven Therapieansatz dar, indem verschiedene schädliche Entstehungsmechanismen des HLM-assoziierten AKI unterbrochen werden. Sie ist darüber hinaus eine günstige, sichere und einfach anwendbare Therapieoption (Haase et al. 2007).

Die Urinalkalisierung mit Natriumbicarbonat schützt vor tubulärer Obstruktion durch Zylinderbildung, indem sie die Entstehung von Methämoglobin aus Hämoglobin hemmt, und sie schützt vor Zellnekrose im proximalen Tubulus durch Reduktion der endozytotischen Aufnahme von Hämoglobin. Da postoperatives AKI nach Operationen unter Einsatz von HLM unter anderem auf eine Hämoglobin-induzierte Pigmentnephropathie zurückgeführt wird (Haase et al. 2007), kann die Gabe von Natriumbicarbonat an dieser Stelle in der Prävention des AKI eine entscheidende Rolle spielen.

Die Bildung freier Radikale wird durch ein saures Milieu, wie es typischerweise im tubulären Urin und im Nierenmark vorliegt, gefördert. Durch den höheren pH-Wert im Extrazellulärraum wird diese Bildung wiederum verhindert, sodass eine Alkalisierung des tubulären Urins mittels Bicarbonat die Nierenschädigung vermindern kann. Die Gabe von Natriumbicarbonat schützt vor der oxidativen Schädigung durch Verlangsamung der pHabhängigen Haber-Weiss-Radikale-Produktion (siehe Anhang) und sie verhindert oxidative Schädigung durch direktes Abfangen von Radikalen wie Peroxynitrit oder anderen Radikalen, die durch Stickstoffmonoxid gebildet werden. So demonstrierten Merten et al. im Tiermodell, dass die Vorbehandlung mit Natriumbicarbonat protektiv im Bezug auf das ischämisch bedingte akute Nierenversagen ist (Merten et al. 2004). Außerdem kommt es auf Grund des alkalischen pH-Wertes durch Natriumbicarbonat zur Präzipitation von freiem Eisen, das auch an der Bildung von Radikalen beteiligt ist, wodurch unlösliches Eisenhydroxid entsteht (Haase et al. 2007). 


\subsection{Prognostische Scores}

\subsubsection{Nutzen prognostischer Scores}

Die höchsten postoperativen Inzidenzen eines AKI treten - wie in den bisherigen Kapiteln beschrieben - nach kardiochirurgischen Eingriffen unter Einsatz der Herz-LungenMaschine auf (Bahar et al. 2005; Mangano et al. 1998; Provenchere et al. 2003), sodass verschiedene Scoring-Systeme beziehungsweise Vorhersagemodelle entwickelt wurden, durch die sich bereits präoperativ anhand bestimmter Risikofaktoren die Wahrscheinlichkeit einer akuten Nierenschädigung nach kardiochirurgischen Eingriffen abschätzen lässt. Diese präzisen Vorhersagen des individuellen Risikos eines Nierenversagens ermöglichen es, gefährdete Patienten mit erhöhtem Risiko zu identifizieren und frühzeitige Therapien gezielt einzusetzen. So können evidenzbasierte Therapieansätze zur Optimierung der Nierenfunktion (Barr und Kolodner 2008; Bellomo 2005; Jones und Lee 2007; Kellum et al. 2005; Merten et al. 2004; Sisillo et al. 2008) selektiv bei gefährdeten Patienten angewendet werden. Dementsprechend kann auch eine risikoadaptierte Planung des anästhesiologischen Vorgehens mit fokussiertem Einsatz nephroprotektiver Maßnahmen und ein begründetes restriktives Vorgehen in Bezug auf perioperative potentiell nephrotoxische Maßnahmen wie beispielsweise Kontrastmitteluntersuchungen erfolgen.

\subsubsection{Charakteristika von prognostischen Scores}

Prognostische Scores unterscheiden sich zunächst in der Zusammensetzung ihrer „Input-Größen“. Die Inputgrößen sind die Prädiktorvariablen beziehungsweise Risikofaktoren, die die „Endpunkt-Größe“ vorhersagen sollen. Man unterscheidet zwischen Scores, die nur präoperative Risikofaktoren berücksichtigen und kombinierten prä-, intra- und postoperativen Scores. Die Scores lassen sich nicht nur durch die Zusammensetzung der Risikofaktoren charakterisieren, sondern auch anhand der unterschiedlichen Gewichtung der einzelnen Risikofaktoren.

Weiterhin muss die Endpunkt-Größe, das vorherzusagende Ereignis, definiert werden. Auch diese variiert in den verschiedenen Scores zur Vorhersage des Nierenversagens. In einigen wird die akute Nierenschädigung beispielsweise noch über die Anwen- 
dung von Nierenersatzverfahren definiert, in anderen bereits über präzise festgelegte Kreatiningrenzwerte.

Darüber hinaus lassen sich Scores durch ihre Gültigkeit, ob sie die Endpunkt-Größe tatsächlich prognostizieren können, charakterisieren. Dabei stellt sich die Frage, wie präzise ein Score in seiner Vorhersagekraft (Kalibrierung oder Kalibration) ist und wie präzise die einzelnen Scorewerte zwischen dem Eintreten und Nicht-Eintreten der EndpunktGröße trennen können (Diskrimination). Nach Entwicklung eines Scores wird der Grad der Genauigkeit, mit dem er das zu untersuchenden Ereignis, das er vorhersagen soll, tatsächlich vorhersagt, über multizentrische Validierungsstudien beurteilt. Liegen die Häufigkeiten des zu untersuchenden Ereignisses innerhalb des prognostizierten Bereichs, spricht man von einer guten Kalibrierung des Scores. Zur Beurteilung der Diskriminationsfähigkeit werden die Sensitivität und Spezifität eines Scores ermittelt.

Das Validierungskollektiv kann zunächst aus dem gleichen Zentrum wie das Evaluierungskollektiv oder einem monozentrischen anderen Kollektiv stammen, sollte aber im Verlauf an mehreren anderen Zentren und im besten Falle innerhalb einer multizentrischen Studie validiert werden.

\subsubsection{Bislang publizierte prognostische Scores}

\subsubsection{Thakar-Score}

Vor dem Hintergrund, dass die Inzidenz des akuten Nierenversagens nach kardiochirurgischen Eingriffen, insbesondere mit Herz-Lungen-Maschinen, sehr hoch ist, entwickelte Thakar einen klinischen Score unter Einbeziehung präoperativer Risikofaktoren, um die individuelle Wahrscheinlichkeit eines postoperativen akuten Nierenversagens bestimmen zu können. Mehr als 30.000 Patienten unterzogen sich an der Cleveland Clinic Foundation in Ohio von 1993 bis 2002 einem kardiochirurgischen Eingriff. An randomisierten 15.838 Patienten entwickelte Thakar ein Risikoabschätzungsmodell und validierte es an 15.839 weiteren Patienten desselben Zentrums. Der Score setzt sich aus 10 Risikofaktoren zusammen, denen in Abhängigkeit ihres relativen Risikos zwischen 1 und 5 Punkte zugeordnet wurden. Der minimale Scorewert beträgt 0 Punkte; bei Präsenz aller Risikofaktoren werden maximal 17 Punkte erreicht. Als Kriterium des akuten Nierenversagens diente die 
Anwendung von Nierenersatzverfahren; dieses wurde durchgeführt bei Hypervolämie, Urämie oder Hyperkaliämie.

Die Validierung des Scores erfolgte sowohl für jeden einzelnen Scorewert, als auch für vier Risikokategorien, in denen Patienten mit Scorewerten von 0 - 2, 3- 5, 6 - 8 und > 9 zusammengefasst wurden, wobei die Grenzwerte für die einzelnen Risikokategorien willkürlich festgelegt wurden. Das Scoring-System von Thakar zeigte nicht nur eine hohe Diskriminationsfähigkeit, sondern auch eine sehr gute Kalibrierung innerhalb der Scorewerte sowie der vier Risikokategorien, sodass von einem präzisen Risikoabschätzungsmodell auszugehen ist, das in der klinischen Praxis hohe Nützlichkeit im Vorbeugen und im Umgang mit akutem Nierenversagen vorweisen kann (Thakar et al. 2005).

Tabelle 1.2 Thakar-Score modifiziert nach Thakar et al. 2005, S. 165. LVEF = Linksventrikuläre Ejektionsfraktion, $\mathrm{COPD}=$ Chronisch obstruktive Lungenerkrankung, IABP = intraaortale Ballonpumpe, $\mathrm{ACB}=$ Aortokoronarer Bypass, $\mathrm{OP}=$ Operation.

\begin{tabular}{|c|c|}
\hline Risikofaktor & Score-Punkte \\
\hline - Weibliches Geschlecht & 1 \\
\hline - Herzinsuffizienz & 1 \\
\hline - $\quad \mathrm{LVEF}<35 \%$ & 1 \\
\hline - COPD & 1 \\
\hline - Insulinpflichtiger Diabetes mellitus & 1 \\
\hline - Wiederholungseingriff & 1 \\
\hline - Notfalleingriff & 2 \\
\hline - Präoperative IABP & 2 \\
\hline \multicolumn{2}{|l|}{ - Art des Eingriffs } \\
\hline O Herzklappen-OP & 1 \\
\hline O $A C B+$ Herzklappen-OP & 2 \\
\hline ○ Sonstige Operation & 2 \\
\hline \multicolumn{2}{|l|}{ - Präoperatives Serumkreatinin } \\
\hline O $1,2 \mathrm{mg} / \mathrm{dl}-2,0 \mathrm{mg} / \mathrm{dl}$ & 2 \\
\hline$\circ \quad \geq 2,1 \mathrm{mg} / \mathrm{dl}$ & 5 \\
\hline
\end{tabular}




\subsubsection{AKICS-Score}

An 603 Patienten wurde 2007 ein weiter Score zur Risikostratifizierung der akuten Nierenschädigung entwickelt: der AKICS-Score (acute kidney injury prediction following elective cardiac surgery), indem nicht nur präoperative Risikofaktoren, sondern auch intra- und postoperative Parameter analysiert wurden. Für folgende Risikofaktoren konnte ein unabhängiger Einfluss auf die Entstehung eines AKI gezeigt werden: Alter über 65 Jahre, präoperatives Kreatinin über $1,2 \mathrm{mg} / \mathrm{dl}$, präoperative Kapillarblut-Glukose über 140 mg/dl, Herzinsuffizienz (Cardiac Failure), kombinierte Eingriffe, HLM-Zeit über zwei Stunden, geringer Cardiac Output und erhöhter zentralvenöser Druck (ZVD). Als Definition der akuten Nierenfunktionsstörung dienten hier die Kriterien Serumkreatinin über $2,0 \mathrm{mg} / \mathrm{dl}$ oder ein Anstieg von über $50 \%$ zum Ausgangskreatinin. Anhand der erreichten Punkte (0-20) werden die Patienten in fünf Kategorien bezüglich ihres Risikos eingeteilt. Im Anschluss daran wurde das entwickelte Scoring-System an einem weiteren Kollektiv, bestehend aus 215 Patienten, validiert. Dort betrug die Inzidenz des AKI 14 \%. Der AKICSScore zeigte eine gute Kalibrierung und ein gutes Diskriminationsvermögen sowohl in dem primär untersuchten Kollektiv als auch im Validierungskollektiv, sodass auch mit dem AKICS-Score ein mögliches AKI in Folge von kardiochirurgischen Einsätzen präzise vorhergesagt werden kann (Palomba et al. 2007).

Tabelle 1.3 AKICS-Score modifiziert nach Palomba et al 2007, S. 627. RF = Risikofaktoren, NYHA = New York Heart Association, ZVD = Zentral-venöser Druck.

\begin{tabular}{lc}
\hline Risikofaktor & Scorepunkte \\
\hline präoperative RF & \\
Präop. Blutglukose $>140 \mathrm{mg} / \mathrm{dl}$ & 1,7 \\
Alter $>65$ & 2,3 \\
Herzinsuffizienz NYHA $>2$ & 3,2 \\
Präop. Serumkreatinin $>1.2 \mathrm{mg} / \mathrm{dl}$ & 3,1 \\
Kombinierter Eingriff & 3,7 \\
intraoperativer RF & \\
HLM-Zeit > 120 min & 1,8 \\
postoperative RF & \\
ZVD $>14 \mathrm{~cm} \mathrm{H}_{2} \mathrm{O}$ & 1,7 \\
Low cardiac output & 2,5 \\
\hline
\end{tabular}




\subsection{Ziel der Untersuchung}

Da sowohl die Entwicklung als auch die Validierung des Thakar-Scores an einem monozentrischen Patientenkollektiv erfolgte, kann die generelle Aussagekraft und Übertragbarkeit erst nach erneuten Validierungen an Patientenkollektiven anderer Zentren beurteilt werden (Thakar et al. 2005). Mit dieser Studie erfolgte die Anwendung auf das Kollektiv in Göttingen und im Anschluss an diese Arbeit ein direkter Vergleich in Regensburg.

Es wurde eine prospektive Beobachtungsstudie mit dem Ziel der Evaluierung und Validierung des Thakar-Scores durchgeführt, anhand dessen sich bereits vor kardiochirurgischen Operationen das Risiko einer postoperativen akuten Nierenfunktionsstörung möglichst präzise voraussagen lässt.

Darüber hinaus ist zu erwähnen, dass die Definition der Outcome-Größe - des akuten Nierenversagens - im Thakar-Score ausschließlich über die Anwendung eines Nierenersatzverfahrens (Dialyse) erfolgte, genauso wie in der zuvor erfolgten retrospektiven Validierungsstudie in Göttingen durch Heise et al. 2010. In der aktuellen Literatur wird jedoch Kritik darüber geäußert, dass in bisherigen gut validierten Scores das Risiko der weniger kritischen AKIN-Stadien nicht bewertet wurde (Huen und Parikh 2012). Demzufolge wurde in der vorliegenden Arbeit die akute Nierenschädigung über die präzise AKINKlassifikation definiert und geprüft, ob der Thakar-Score das Vermögen besitzt, anstelle des Risikos für ein schweres AKI mit der Notwendigkeit einer Dialyse, bereits mildere Verlaufsformen eines AKI präzise vorherzusagen.

\subsubsection{Validität des Thakar-Scores in einem Göttinger Kollektiv unter Verwendung der AKIN-Kriterien}

Nachdem die individuellen Scorepunkte nach Thakar für jeden Patienten ermittelt wurden, erfolgte die Validierung des Thakar-Scores. „Validität bezeichnet die Menge der zutreffenden Schlussfolgerungen, die aus einem Testergebnis gezogen werden können“ (Moosbrugger 2012, S. 429). Hierbei erfolgte die Prüfung der Gültigkeit des Thakar-Scores als Vorhersagemodell für akute Nierenschädigungen für ein übertragbares Kollektiv, indem die Kalibrierung und das Diskriminationsvermögen im Göttinger Kollektiv bestimmt 
wurden. Die Kalibrierung zeigt an, wie präzise der Score das Ereignis AKI prognostizieren kann. Hierzu werden die tatsächlichen AKI-Inzidenzen der jeweiligen Scorepunkte und Score-Kategorien mit den 95\%-Konfidenzintervallen der von Thakar prognostizierten Inzidenzen verglichen. Die Diskrimination ist die Trennschärfe eines Scores, die angibt, wie genau die verschiedenen Scorewerte die Patienten mit und ohne AKI voneinander „trennen“ können. Hierzu werden Sensitivität und Spezifität ermittelt. Eine hohe Diskriminationsfähigkeit des Scores liegt vor, wenn möglichst viele Scorewerte richtig-positive Ergebnisse (Sensitivität) beziehungsweise richtig-negative Ergebnisse (Spezifität) vorweisen.

\subsubsection{Modifikation des Thakar-Scores durch Re-Evaluation}

Nachdem die Validierung des Thakar-Scores erfolgte, wurde untersucht, ob durch Vornahme von Modifikationen am Original-Score die Vorhersagekraft des Scores verbessert werden kann. Diese Modifikationen beinhalteten eine Analyse der präoperativen Risikofaktoren von Thakar hinsichtlich der quantitativen Ausprägung ihres unabhängigen Einflusses auf die AKI-Inzidenz. Weiterhin wurde überprüft, ob sich die Diskriminationsfähigkeit verbessern lässt, wenn die Gewichtungen der einzelnen Risikofaktoren angepasst werden. Der quantitative Einfluss aller Risikofaktoren des Scores wurde hierfür neu berechnet und entsprechend veränderte Scorepunkte wurden vergeben. Eine erneute Validitätsprüfung des re-evaluierten modifizierten Scores erfolgte wie oben beschrieben anhand seiner Kalibrierung und Diskriminationsfähigkeit. Die daraus ermittelte Vorhersagekraft des modifizierten Scores wurde mit dem Original-Score von Thakar verglichen.

\subsubsection{Modifikation des Thakar-Scores durch Hinzunahme intraoperati- ver Risikofaktoren}

Neben dem Einfluss präoperativer Risikofaktoren konnten zahlreiche intraoperative Einflussgrößen identifiziert werden (Abu-Omar und Ratnatunga 2006; Haase und Shaw 2010; Karkouti et al. 2005; Oyar et al. 2012; Parolari et al. 2012), die sich auf die Inzidenz eines AKI auswirken können. So stellt sich die Frage, wie sich ein neuer Score neben der Modifikation der Orginal-Risikofaktoren von Thakar durch Hinzunahme von intraoperativen Risikofaktoren verhält. Können Kalibrierung und Diskrimination noch weiter verbes- 
sert werden? Hierfür wurden folgende weitere Parameter erhoben: HLM-Zeit, Aortenklemmzeit, Anzahl der verabreichten Erythrozytenkonzentrate, Fresh-Frozen-Plasma (FFP) und Thrombozytenkonzentrate sowie intraoperative Hämofiltration. Es wurde untersucht, welche dieser Parameter einen unabhängigen Einfluss ausüben und wie ausgeprägt dieser Einfluss auf die Entstehung eines AKI ist. Darauffolgend wurde analog zu Thakar der erweiterte, modifizierte Score entwickelt, der erneut wie oben beschrieben validiert wurde.

\subsubsection{Auswirkung einer Therapie mit Natriumbicarbonat}

Haase et al. postulierten, dass die intravenöse Applikation von Natriumbicarbonat eine günstige, sichere und einfach durchführbare Methode in der Prävention des Nierenversagens nach kardiochirurgischen Eingriffen darstellt. Die Urinalkalisierung mit Natriumbicarbonat stellt einen vielversprechenden protektiven Therapieansatz dar, indem verschiedene schädliche Entstehungsmechanismen des HLM-assoziierten AKI unterbrochen werden (Haase et al. 2009).

Während der Patienten-Rekrutierungsphase dieser Arbeit nahm die Abteilung Anästhesie und Intensivmedizin des Universitätsklinikums Göttingen - basierend auf den Resultaten von Haase et al. - die intravenöse Gabe von 8,4\%iger NatriumbicarbonatInfusionslösung in die interne Standard-Handlungsempfehlungen (Standard Operation Procedure) auf. Demzufolge erhielten ab dem 09.08.2010 alle Patienten, die unter Einsatz einer HLM operiert wurden, diese Therapiemaßnahme.

So wurde in dieser Arbeit die folgende Frage untersucht: Wirkt sich die Gabe von Natriumbicarbonat tatsächlich protektiv auf die Entwicklung eines AKI nach kardiochirurgischen Eingriffen aus? Da alle sonstigen relevanten Risikofaktoren für das Auftreten eines AKI innerhalb dieser Studie erfasst wurden, konnte der tatsächliche Effekt des Natriumbicarbonats evaluiert werden, indem die Inzidenz eines AKI unter 224 Patienten, die eine Natriumbicarbonattherapie erhielten (im Folgenden als „NaHCO $3-\mathrm{Gruppe}$ bezeichnet) mit einer Kontrollgruppe aus ebenfalls 224 Patienten verglichen wurde. Durch Errechnen der individuellen Scorewerte jedes Patienten konnte bei gleich verteilten Scorewerten eine gleichmäßige Risikoverteilung zwischen der $\mathrm{NaHCO}_{3}-\mathrm{Gruppe}$ und der Kontrollgruppe sicher gestellt werden und ein Einfluss durch unterschiedlich verteilte Risikofaktoren ausgeschlossen werden. Außerdem wurde durch Erheben der intraoperativen 
freien Hämoglobinwerte das Ausmaß der Hämolyse evaluiert und ebenfalls deren Verteilung in beiden Kollektiven überprüft. Auf diese Weise konnte direkt untersucht werden, ob (bei gleichen Risikokonstellationen) eine Therapie mit Natriumbicarbonat mit einer geringeren Inzidenz der akuten Nierenschädigung einhergeht. Auch hier diente die AKINKlassifikation als Kriterium der akuten Nierenschädigung. 


\section{MATERIAL und METHODEN}

\subsection{Studiendesign und Studienablauf}

Die Untersuchung wurde als prospektive Beobachtungsstudie durchgeführt. 1.007 Patienten der operativen Intensivstation 0118 des Universitätsklinikums Göttingen wurden nach Genehmigung der Ethik-Kommission und Patientenaufklärung mit schriftlicher Einverständniserklärung in die Studie eingeschlossen. Nach Studieneinschluss erfolgte anhand der Patientenunterlagen zunächst die Überprüfung der präoperativen Risikofaktoren. Um den Einfluss intraoperativer Parameter für das Auftreten einer Nierenfunktionsstörung beurteilen zu können, wurden die vermuteten Haupt-Risikofaktoren (Dauer der Herz-Lungen-Maschine, Aortenklemmzeit, Anzahl transfundierter Blutprodukte, intraoperative Hämofiltration sowie intraoperative Konzentration an freiem Hämoglobin) ebenfalls erfasst. Anschließend wurde für jeden Patienten der individuelle Scorewert nach Thakar bestimmt.

Für die Dauer von sechs Tagen wurde täglich anhand des Serumkreatinins und der Diuresemenge die AKIN-Klassifikation vorgenommen. Es wurde jeweils die maximal erreichte AKIN-Klasse innerhalb der sechs postoperativen Tage eines Patienten gewertet. Die tägliche Bestimmung des Serumkreatinins gehört sowohl auf der Intensivstation als auch auf den chirurgischen Normalstationen zur klinischen Routine, sodass keine studienspezifischen Blutentnahmen oder Laboruntersuchungen durchgeführt wurden.

Nach Abschluss der Patientenrekrutierung und Ermittlung des maximal erreichten AKIN-Stadiums jedes Patienten erfolgte die Aufteilung der Göttinger Patienten gemäß der AKIN-Stadien in je eine Gruppe ohne (AKIN 0) beziehungsweise mit akuter Nierenschädigung (AKIN 1-3).

\subsection{Studienzeitraum}

Der Studienzeitraum mit Patientenrekrutierung erstreckte sich von Juli 2009 bis Februar 2011. 


\subsection{Patienten}

\subsubsection{Patientenkollektiv}

Die Untersuchung wurde an Patienten des Universitätsklinikums Göttingen durchgeführt, die sich einem kardiochirurgischen Eingriff unter Einsatz eines kardiopulmonalen Bypasses unterzogen und einer Studienteilnahme zustimmten. Die durchgeführten Operationen waren Aortokoronare Bypass-Operationen mit oder ohne Ersatz beziehungsweise Rekonstruktion der Herzklappen, alleinige Herzklappenoperationen, oder sonstige Operationen unter Einsatz der Herz-Lungen-Maschine.

\subsubsection{Patienteninformation und Einwilligungserklärung}

Es erfolgte die schriftliche Einwilligung an der Teilnahme der Studie von allen Patienten. Die Patienten wurden über den Inhalt, die Hintergründe, den Ablauf und die Perspektive der Studie, sowie über die Möglichkeit eines Widerrufs der Einwilligung aufgeklärt. Des Weiteren wurde den Patienten ein zweiseitiges Informationsblatt (siehe Anhang) über die Studie ausgehändigt und ausreichend Zeit zur Überlegung und zur Vorbereitung von Fragen ermöglicht. Mit der Einwilligung zur Teilnahme an der Studie erklärten sich die Patienten damit einverstanden, ihre persönlichen Daten in pseudonymisierter Form im Rahmen der Studie zu Verfügung zu stellen, dass ihre Krankenanamnese retrospektiv erhoben und im Rahmen der Studie genutzt wird und oben genannte Parameter prospektiv bestimmt werden.

\subsubsection{Ausschlusskriterien}

Patienten wurden nicht in die Studie eingeschlossen, wenn ein oder mehrere der folgenden Kriterien vorlagen:

1. Alter $<18$ Jahre

2. Vorliegen einer bereits präoperativ bestehenden terminalen Nierenfunktionsstörung mit Dialysepflicht

3. Kardiochirurgischer Eingriff ohne Einsatz der HLM

a. Transapikale Herzklappenoperationen

b. Off-pump-Operationen 
4. Herztransplantationspatienten

5. Präoperative ECMO (extrakorporale Membranoxygenierung)

6. Präoperatives Tracheostoma oder mechanische Ventilation

7. ICD-Implantation

8. LVAD (left ventricular assist devices): Membranpumpen oder axiale Rotationspumpen zur mechanischen Kreislaufunterstützung

9. Teilnahme an der Studie vom Patienten abgelehnt

10. Patienten nicht einwilligungsfähig

11. Versterben des Patienten im Operationssaal

12. Inkomplette Datenerfassung.

Dabei konnten Personen mehr als einmal erfasst werden, wenn sie innerhalb des Studienzeitraums mehr als einmal einen operativen Eingriff unter Einsatz der HerzLungen-Maschine hatten. Eine erneute schriftliche Aufklärung der Patienten erfolgte in diesem Fall nicht.

\subsection{Datensammlung, Archivierung und Datenschutz}

Die Datenerfassung, Datenverwaltung und Archivierung erfolgte im Tabellenkalkulationsprogramm Microsoft Excel.

Die Erfassung und Archivierung der Daten erfolgte pseudonymisiert, das heißt ohne Namen, Patientennummern oder sonstige Angaben, die eine nachträgliche Identifikation der Patienten zulassen könnten. Um bei mehreren, parallel untersuchten Patienten eine eindeutige Zuordnung während der Datenerfassungsperiode zu ermöglichen, wurden lediglich die Initialen gespeichert.

\subsection{Datenerfassung}

Die Daten der Patienten wurden aus den Krankenakten erhoben: Dazu dienten das Anästhesie-OP-Protokoll, das chirurgische OP-Protokoll, Kardiotechniker-Protokoll, präoperative Lungenfunktionsdiagnostik, Herzkatheter und Herzechographie-Befund, Arzt- 
briefe und daneben die klinikinternen Programme IXSERV, GISI und ICIP und die Rücksprache mit den jeweiligen behandelnden Ärzten.

Folgende Daten wurden erfasst:

- persönliche Patientendaten:

- teilanonymisierter Name: über ein Kürzel bestehend aus jeweils dem ersten Buchstaben des Vornamens und des Nachnamens

- Geburtsdatum

- Alter

- Geschlecht

- Gewicht

- Operationsdatum

- Art des Eingriffs:

- $\mathrm{ACB} / \mathrm{ACV} B$

- Herzklappenoperation

- Kombinierte $A C B / A C V B$ und Herzklappeneingriff

- Sonstige Art von Eingriff

- Primär-Operation oder Re-Operation

- Elektiv-Operation oder Nicht-Elektiv-Operation

- Weitere präoperative Risikofaktoren nach Thakar:

- Linksventrikuläre Ejektionsfraktion

- NYHA-Stadium 0 bis IV

- COPD

- Insulinpflichtiger Diabetes mellitus

- Präoperativer Kreatininwert

- Präoperativer Einsatz einer Intraaortalen Ballonpumpe

- Intraoperative Risikofaktoren:

- Anzahl der Blutprodukte, unterschieden zwischen Erythrozytenkonzentraten, Fresh-Frozen-Plasma und Thrombozytenkonzentraten

- Dauer des HLM-Einsatzes

- Aortenklemmzeit

- Hämofiltration an der HLM, ggfs. entzogenes Volumen. 
Darüber hinaus wurden bei 448 Patienten die intraoperativen freien Hämoglobinwerte und die Gabe von Natriumbicarbonat-Puffer-Infusionslösungen intra- und/oder postoperativ erfasst. Die intraoperativen freien Hämoglobinwerte wurden zu mehrfachen Messzeitpunkten erfasst: der Hämoglobin-Ausgangswert nach Narkoseeinleitung, der PräBypass-Hämoglobinwert nach vollständigem Anschluss an die noch stehende HLM sowie weitere Abnahmen während des HLM-Einsatzes und 15 Minuten nach HLM-Beendigung.

Die Definition und Graduierung des akuten Nierenversagens erfolgte über die AKINKlassifikation. Hierfür wurden am Operationstag und den ersten fünf postoperativen Folgetagen die Diuresemenge und Kreatininwerte erfasst und das jeweilige AKIN-Stadium ermittelt. Es wurde jeweils die maximal erreichte AKIN-Klasse eines Patienten gewertet.

\subsection{Statistische Verfahren}

Die statistische Datenanalyse wurde mit Microsoft Excel, Statistika und MedCalc durchgeführt. $\chi 2$-Test, Kolmogorow-Smirnow-Test, t-Test für unverbundene Stichproben, Mann-Whitney-Test, logistische Regressionsanalysen und ROC-Analysen wurden mit den Statistikprogrammen Statistika und MedCalc durchgeführt. Die Grafiken wurden mit Microsoft Word 2007, Microsoft Excel 2007 und Statistika erstellt.

Die Daten wurden zunächst auf Plausibilität überprüft und anschließend einer Analyse deskriptiver Statistik unterzogen. Die formale Prüfung beinhaltete die Prüfung auf korrekte Formatierung der Daten. Die inhaltliche Prüfung umfasste eine Kontrolle auf Plausibilität, auf realistische Werte der Variablen, korrekte Berechnung von Scorewerten und korrekte Zuteilung entsprechend des Klassifikationssystems AKIN. Alle Testungen erfolgten zweiseitig, das heißt Abweichungen von einem Sollwert waren sowohl nach oben als auch nach unten gleich gewichtet.

\subsubsection{Deskriptive Statistik}

Zunächst wurde für die Parameter eine deskriptive Statistik erstellt, um die Zusammensetzung der Untersuchungsgruppe hinsichtlich der verschiedenen Risikofaktoren und der AKIN-Stadien zu charakterisieren. „Die deskriptive Statistik stellt Analysetechniken zur Verfügung, die der explorativen Beschreibung und Charakterisierung empirischer Daten in 
Form ihrer Verteilung, Lage und Streuung dienen“ (Pospeschill 2006, S. 9). Hierbei wurden Mittelwerte, Median, Interquartil-Bereich (IQR), Varianz und Standardabweichungen im 95\%- Konfidenzintervall bestimmt, wobei die Varianz und Standardabweichung Maße für die Streuung sämtlicher Werte einer Variablen um ihren Mittelwert sind. Die Varianz ist definiert als „Summe der quadrierten Abweichungen aller Messwerte vom arithmetischen Mittel, dividiert durch die Anzahl aller Messwerte" (Bortz 2005, S. 41). Die Standardabweichung einer Variablen ergibt sich aus der Quadratwurzel der berechneten Varianz. „Das Konfidenzintervall kennzeichnet denjenigen Bereich eines Merkmals, in dem sich 95\% aller möglichen Populationsparameter befinden, die den empirisch ermittelten Stichprobenkennwert erzeugt haben können“ (Bortz 2005, S.102). Der Interquartilbereich gibt den Werte-Bereich zwischen dem $75 \%$ - und $25 \%$ - Quantil an, in dem sich $50 \%$ aller Werte befinden.

\subsection{2 Überprüfung der Validität des Thakar-Scores am Göttinger Kol- lektiv}

Korreliert der Test mit einem sogenannten Außenkriterium, einem in der Zukunft liegenden Ereignis, wie das AKI, so spricht man von Vorhersagevalidität oder prognostischer Validität (Kubinger 2003). „Validität bezeichnet die Menge der zutreffenden Schlussfolgerungen, die aus einem Testergebnis gezogen werden können“ (Moosbrugger 2012, S. 428).

Zur Validierung des Thakar-Scores gehören die Überprüfung der Kalibration und der Diskriminationsfähigkeit. Die Kalibration trifft eine Aussage darüber, wie präzise die tatsächliche Inzidenz des AKI anhand der Scorewerte vorausgesagt werden kann. Die Diskrimination untersucht die Fähigkeit, anhand des Scorewertes Patienten mit und ohne AKI voneinander zu unterscheiden beziehungsweise zu „trennen“, das heißt die Trennschärfe. Der Score kann als valide und übertragbar gelten, wenn er im Validierungskollektiv eine gute Kalibrierung und ein gutes Diskriminationsvermögen aufweist, was im Folgenden erläutert wird.

Als Basis der statistischen Analyse wurden den Patienten die kontinuierliche Größe „Scorewert" und die dichotome Endpunkt-Größe „AKI“ zugeordnet. 


\subsubsection{Kalibrierung}

Zunächst wurde für jeden Patienten nach vollständigem Erfassen aller Parameter gemäß dem Thakar-Score der jeweilige präoperative summierte Scorewert ermittelt (siehe Kapitel 1.7.3.1). Die Patienten wurden in Gruppen mit gleichen präoperativen Scorewerten (0-17) eingeteilt und den vier Risikogruppen analog zu den Risikokategorien nach Thakar (0-2; 3-5; 6-8; > 9 Scorepunkte) zugeordnet (Thakar et al. 2005).

Für jeden Score-Wert (0-17) und für jede Risikokategorie (1-4) wurde daraufhin die AKI-Inzidenz ermittelt. Anschließend wurde die tatsächliche Inzidenz in Göttingen mit der Inzidenz, die Thakar et al. für den jeweiligen Scorewert prognostizierten, verglichen. Liegen die Werte des Göttinger Validierungskollektivs innerhalb der $95 \%$ Konfidenzintervalle der von Thakar prognostizierten Inzidenzen, so weist der Score eine gute Kalibrierung auf.

\subsubsection{Diskrimination}

Im zweiten Schritt wurde die Diskriminationsfähigkeit des Scores beurteilt. Hierbei wird, wie oben beschrieben, die Trennschärfe untersucht; wie "treffsicher" ist der Score, zwischen „kranken“ und „gesunden“ Patienten zu trennen? Hierfür wurden die Sensitivität und Spezifität in Abhängigkeit von verschiedenen Cut-Off-Werten mit Hilfe der Receiver-Operating-Characterisitc-Analyse (ROC) ermittelt, die die Trennschärfe zwischen richtig-positiven ( $R P$, richtig erkannte Kranke) und falsch-positiven (FP, krank erklärte, übersehene Gesunde) beziehungsweise richtig-negativen (RN, erkannte Gesunde) und falschnegativen (FN, übersehene Kranke) angeben.

Tabelle 2.1 Sensitivität und Spezifität

\begin{tabular}{|c|c|c|}
\hline & "krank" & "gesund“ \\
\hline Test positiv & RP & FP \\
\hline Test negativ & FN & RN \\
\hline
\end{tabular}

\subsection{Sensitivität}

Die Sensitivität eines Tests gibt den Anteil positiver Testergebnisse $(T+)$ unter den Kranken $(\mathrm{K}+)$ an. Sie wird also als Quotient aus richtig positiven Testergebnissen und tat- 
sächlich Kranken berechnet. Die tatsächlich Kranken setzen sich aus der Summe von richtig positiven und falsch negativen Testergebnissen zusammen. Je höher die Sensitivität eines Tests ist, desto sicherer erfasst ein Test eine Erkrankung und kein kranker Patient wird übersehen. In anderen Worten bedeutet ein negativer Test bei hoher Sensitivität, dass der Patient sicher gesund ist. Abstrakt wird die Sensitivität wie folgt beschrieben: Die Sensitivität eines diagnostischen Tests $\mathrm{P}(\mathrm{T}+\mid \mathrm{K}+)$ gibt die Wahrscheinlichkeit dafür an, dass bei Vorliegen eines Sachverhaltes $(\mathrm{K}+)$ das Testergebnis positiv ausfällt $(\mathrm{T}+)$ (Bortz 2005; Hilgers et al. 2007; Kubinger 2003; Moosbrugger 2012).

\subsection{Spezifität}

Die Spezifität gibt den Anteil negativer Testergebnisse (T-) unter den Gesunden (K-) an, dementsprechend die Anzahl korrekter Ablehnung. Die abstrakte Definition der Spezifität lautet: Die Spezifität eines Tests $\mathrm{P}(\mathrm{T}-\mid \mathrm{K}-)$ gibt die Wahrscheinlichkeit dafür an, dass bei Nicht-Vorliegen eines Sachverhalts (K-) das Testergebnis negativ ausfällt (T-). Sie wird als Quotient aus richtig negativen Testergebnissen und tatsächlich Gesunden berechnet. Die tatsächlich Gesunden setzen sich zusammen aus der Summe aus falsch positiven (krank erklärte, übersehene Gesunde) und richtig negativen (richtig erkannte Gesunde). Eine hohe Spezifität heißt, dass kein gesunder Patient fälschlicherweise als krank bezeichnet wird und wenn das Testergebnis positiv ausfällt, der Patient sicher krank ist (Bortz 2005; Hilgers et al. 2007; Moosbrugger 2012; Sachs und Hedderich 2009).

\subsection{ROC-Analyse}

Die ROC-Analyse stellt ein statistisches Verfahren dar, das aus der Signalentdeckungstheorie der Psychophysik von Green und Swets aus dem Jahr 1966 stammt, um bei einem diagnostischen Test mit nicht-dichotomen Variablen die beschriebenen Testgütekriterien Sensitivität und Spezifität beurteilen zu können (Moosbrugger 2012).

Die Scorewerte, deren Sensitivität und Spezifität bestimmt werden sollen, stellen eine solche nicht-dichotome, kontinuierliche Variable dar. Da kontinuierliche Größen prinzipiell keinen „natürlichen“ Trennwert besitzen, ist zunächst eine Überführung dieser Variablen in eine binäre Aussage notwendig. Es wird ein sogenannter Cut-off-Wert bestimmt, um die Variable zu dichotomisieren. Das bedeutet, dass sie jetzt nur noch zwei Ausprägungen einnehmen kann: eine unterhalb des Cut-off-Wertes und eine oberhalb 
des Cut-off-Wertes. Dieser Cut-off-Wert ist frei wählbar und entscheidet maßgeblich über Sensitivität und Spezifität.

Da Sensitivität und Spezifität von diesem Trennwert beziehungsweise Schwellenwert abhängig sind, ist der zur Fallunterscheidung verwendete Trennwert der Variablen so festzulegen, dass Trefferquote und Quote korrekter Ablehnungen maximiert werden. So wird eine maximale Trennschärfe erreicht. Ein niedrig gewählter Cut-off-point bewirkt eine hohe Sensitivität bei geringer Spezifität. Umgekehrt führt ein hoher Cut-off-point zu einem Test mit geringer Sensitivität und hoher Spezifität.

In der ROC-Analyse wird für jeden möglichen Cut-off-Point die dazugehörige Sensitivität und Spezifität errechnet. Anschließend werden die Wertepaare von Sensitivität und Spezifität für alle möglichen Cut-Off-Points innerhalb des Messbereiches graphisch dargestellt, woraus die ROC-Kurve resultiert. (1-Spezifität) wird entlang der Abszisse, Sensitivität entlang der Ordinate aufgetragen. Der diagnostische Test zeigt dann Trennschärfe, wenn sich die Kurve signifikant von der Diagonalen unterscheidet. Je größer der Abstand der ROC-Kurve von der Diagonalen ist, desto höher ist die Trennschärfe des Tests. Im Idealfall liegt die Kurve auf der linken beziehungsweise oberen Begrenzungsseite des umschließenden Quadrates. Liegt die Kurve auf der Diagonalen, bedeutet es, dass die Summe aus Sensitivität und Spezifität für alle Schwellenwerte gleich groß ist und es läge ein reiner Zufallsbefund vor; der diagnostische Test hätte keine Aussagekraft. Mit demjenigen Trennwert, für den die Summe von Sensitivität und Spezifität am größten wird, ist die Trennschärfe zwischen beiden Kollektiven am größten. „Rechnerisch lässt sich dieser Punkt über den Youden-Index bestimmen, der als (Sensitivität + Spezifität - 1) so gebildet wird, dass er Werte zwischen 0 und 1 annimmt" (Moosbrugger 2012, S. 186). Der entsprechende Punkt auf der ROC-Kurve ist derjenige, an dem das Lot auf die Hauptdiagonale den größten Abstand aufweist. Mit Hilfe der graphischen ROC-Analyse können jedoch auch Trennwerte festgelegt werden, die ein anderes Optimierungsverhältnis haben als das aus dem Youden-Index resultierende maximale. Beispielsweise kann ein niedriger Schwellenwert mit niedriger Spezifität, aber hoher Sensitivität zweckmäßiger sein und damit den optimalen Schwellenwert darstellen (Moosbrugger 2012; Sachs und Hedderich 2009).

Exakt quantifiziert wird die Trennschärfe beziehungsweise die Güte des Tests über die Berechnung der Fläche unter der ROC-Kurve: „Fläche unter der Kurve“ oder „Area 
under the curve" (AUC). Dabei kann sie Werte zwischen 0,5 und 1 annehmen, wobei ein höherer Wert die bessere Trennschärfe anzeigt (Sachs und Hedderich 2009).

Die Validierung des Scores am Göttinger Kollektiv erfolgte durch Errechnen der AUC, der optimalen Sensitivität und Spezifität, und dem Vergleich mit dem Ergebnis am Kollektiv von Thakar.

\subsubsection{Re-Evaluation des Thakar-Scores am Göttinger Kollektiv}

Wie im Kapitel 1.8 beschrieben, wurde untersucht, ob sich die Kalibrierung und Diskriminationsfähigkeit des Scores durch Vornahme von Modifikationen am Original-Score verbessern lässt. Hierfür wurden die einzelnen präoperativen Risikofaktoren von Thakar hinsichtlich ihres Einflusses auf die AKI-Inzidenz im Göttinger Kollektiv mittels monovariater Analyse überprüft und deren unabhängiger und quantitativer Einfluss mittels multivariater Analyse bestimmt.

\subsubsection{Monovariate Analyse}

Ist ein Risikofaktor in den beiden Outcome-Gruppen AKIN 0 und AKIN 1-3 gleich verteilt beziehungsweise ausgeprägt, kann davon ausgegangen werden, dass er keinen Einfluss auf das Outcome hat. Unter dieser Annahme wurde zunächst die Verteilung der Risikofaktoren auf dem Signifikanzniveau $p \leq 0,05$ überprüft, wobei $p \leq 0,01$ ein sehr beziehungsweise hoch signifikantes Ergebnis darstellt. Hierzu dienten in Abhängigkeit der Parameter folgende Tests: $\mathrm{Chi}^{2}$-Test für kategorische Parameter, $\mathrm{t}$-Test für kontinuierliche normalverteilte Parameter und der Mann-Whitney-U-Test für kontinuierliche nichtnormalverteilte Variablen.

\subsection{Chi²-Test $^{2}$}

Der $\mathrm{Chi}^{2}$-Test oder $\mathrm{\chi}^{2}$ - Test wird zur Analyse von kategorischen Parametern im Nominalskalenniveau (beispielsweise Geschlecht) durchgeführt. Er untersucht die unterschiedliche Verteilung von nominalskalierten Variablen in den Untersuchungsgruppen. Mit Hilfe des $\chi 2$-Tests wird der Unterschied zwischen den beobachteten und den nach der Nullhypothese zu erwarteten Häufigkeiten bestimmt und berechnet, ob dieser Unterschied signifikant ist (Leonhart 2004). 
Die Häufigkeiten der Variablen werden in sogenannten Kontingenztafeln angeordnet. Die einfachste Form, um Unterschiede verschiedener Merkmalsausprägungen zu untersuchen, ist die 2x2-Kontingenztafel: die Vierfeldertafel (Leonhart 2004; Pospeschill 2006). Eine Variable mit jeweils zwei Ausprägungen „vorhanden“ / „nicht vorhanden“ (beispielsweise „weibliches Geschlecht“ „vorhanden“ / „nicht vorhanden“) wird bezüglich ihrer Verteilung oder Häufigkeit in den zwei Ausprägungen einer anderen Variable (AKIN 0 und AKIN 1-3) untersucht.

\subsection{Normalverteilung der kontinuierlichen Variablen}

Die Normalverteilungsannahme der kontinuierlichen Variablen kann mit dem Kolmogorow-Smirnow-Test geprüft werden. Liegt eine Normalverteilung vor, kann der t-Test für unverbundene Stichproben angewendet werden. Bei kontinuierlichen Parametern, die keiner Normalverteilung unterliegen, kommt der Wilcoxon-Mann-Whitney-Test für unabhängige Stichproben zum Einsatz.

\subsection{T-Test}

Der Zweistichproben-t-Test ist ein Signifikanztest zum Testen von Hypothesen über Erwartungswerte zweier unabhängiger Stichproben aus normalverteilten Grundgesamtheiten. Das Ergebnis ist die Irrtumswahrscheinlichkeit p, mit der die Nullhypothese der Annahme gleicher Mittelwerte in den Gruppen abgelehnt werden kann. Er prüft infolgedessen anhand der Mittelwerte zweier Stichproben, ob die Mittelwerte zweier Grundgesamtheiten einander gleich sind, gegen die Alternativhypothese, dass einer der Mittelwerte kleiner ist als der andere (Sachs und Hedderich 2009).

\subsection{Mann-Whitney-U-Test}

Alternativ wird in dieser Studie der Mann-Whitney-U-Test für nicht-normalverteilte, unverbundene Stichproben verwendet. Der Mann-Whitney-U-Test - oder auch WilcoxonMann-Whitney-Test genannt - gehört zu den nicht-parametrischen Tests (das heißt für nicht-normalverteilte Variablen) und kommt zum Einsatz, um zwei unabhängige Stichproben hinsichtlich ihrer zentralen Tendenz (Maß für das Zentrum der Verteilung) in einem mindestens ordinalskalierten Merkmal (Nominaldaten in Rangreihen) zu vergleichen (Leonhart 2004). Er dient der Bestimmung von Mittelwertunterschieden zwischen verschiedenen Untersuchungsgruppen beziehungsweise unterschiedlichen Verteilungen der Variablen innerhalb der Gruppen. Es wird untersucht, ob dieser berechnete Unterschied be- 
ziehungsweise die Teststatistik auch signifikant ist. Im Gegensatz zum t-Test, der metrischskalierte Variablen verlangt, müssen die Variablen dabei nicht normalverteilt sein und nur Ordinalskalenniveau besitzen. Der U-Test wird als Rangsummentest durchgeführt, das heißt die Berechnung der Teststatistik basiert auf dem Vergleich von Rangreihen. Die Rangreihen würden sich in beiden Gruppen gleichmäßig verteilen, wenn sich die zentrale Tendenz der Gruppen nicht unterscheidet. Durch Addition der Rangplätze erhält man eine Rangsumme. Anschließend wird die Prüfgröße $U$ bestimmt, indem ausgezählt wird, wie häufig ein Rangplatz in der einen Stichprobe größer ist als die Rangplätze in der anderen Gruppe; analog wird mit allen Rangplätzen verfahren (Bortz und Schuster 2010; Pospeschill 2006).

\subsubsection{Multivariate Analyse}

Nachdem die Parameter auf eine signifikant unterschiedliche Verteilung in beiden Outcome-Gruppen überprüft wurden, wurden diejenigen, für die eine unterschiedliche Verteilung gezeigt werden konnte, einer multivariaten Analyse unterzogen. Hierbei wird untersucht, welche Variablen die Entstehung eines AKI unabhängig von anderen Variablen beeinflussen. So werden die signifikant unabhängigen Einflussgrößen von den nichtunabhängigen herausgearbeitet. In Abhängigkeit des Skalenniveaus der Variablen wird die lineare oder logistische Regressionsanalyse angewendet. In dieser Studie wurde die in den nächsten Punkten beschriebene logistische Regression durchgeführt.

\subsection{Regressionsanalyse}

Die Regressionsanalyse ist ein Analyseverfahren, das „sowohl für die Beschreibung und Erklärung von Zusammenhängen als auch für die Durchführung von Prognosen“ (Backhaus et al. 2008, S. 12), mit welcher Wahrscheinlichkeit bestimmte Ereignisse in der Zukunft eintreten, große Bedeutung besitzt. Sie dient der Analyse von Beziehungen zwischen einer abhängigen, der Kriteriumsvariablen, und einer oder mehreren unabhängigen Prädiktorvariablen (Backhaus et al. 2008; Bortz und Schuster 2010). Es lassen sich „Hypothesen über Wirkungsbeziehungen prüfen“ und „Prognosen erstellen“ (Backhaus et al 2008, S. 12). „Mit Hilfe der Regressionsanalyse können derartige Beziehungen quantifiziert und damit weitgehend exakt beschrieben werden" (Backhaus et al. 2008, S. 12). Im Unterschied zur Korrelation dient die Regression also nicht primär zur Beschreibung von 
Zusammenhängen, sondern der Vorhersage einer Kriteriumsvariablen anhand von Prädiktoren (Leonhart 2004).

\subsection{Logistische Regression}

Bei der logistischen Regressionsanalyse wird die Zugehörigkeits-Wahrscheinlichkeit der (in der Regel dichotomen) abhängigen Variablen in Abhängigkeit von einer oder mehreren unabhängigen Variablen zu einer Kategorie bestimmt (Bortz 2010). „Da zur Schätzung der Eintrittswahrscheinlichkeiten der Kategorien der abhängigen Variablen auf die (s-förmige) logistische Funktion zurückgegriffen wird, gehört dieses Verfahren zu den nicht-linearen Analyseverfahren“ (Backhaus et al. 2008, S. 14). Die logistische Regressionsanalyse kann bei Variablen mit verschiedenen und auch gemischten Skalenniveaus eingesetzt werden. Im Gegensatz zur linearen Regression weist die abhängige Variable kein metrisches Skalenniveau auf, sondern ist eine kategoriale Variable mit nominalem Skalenniveau. Diese kategoriale Variable besitzt in der Regel zwei Ausprägungen (binär), kann aber auch multinominal (mehr als zwei Ausprägungen) sein. Die unabhängigen Variablen werden als Faktoren bezeichnet. Als unabhängige Variable können sowohl nominalskalierte kategoriale Variablen als auch metrische Variablen berücksichtig werden, wobei kategoriale unabhängige Variablen in binäre Variablen, die nur die Werte 0 oder 1 annehmen können, überführt werden müssen (Backhaus et al. 2008). Die unabhängigen Prädiktorvariablen werden zur Vorhersage eingesetzt und sind die nicht-beeinflussbaren Parameter (präoperative Risikofaktoren). Die abhängige Variable (AKI) beschreibt die Auswirkungen des Einflusses der unabhängigen Variablen und soll vorhergesagt werden (Bortz und Schuster 2010).

\subsection{Regressionskoeffizient}

Anhand der multiplen Regressionsanalyse ist es möglich, Beziehungen zwischen mehreren Prädiktorvariablen und einer Kriteriumsvariablen zu untersuchen. Das Ergebnis ist die Regressionsfunktion zur Vorhersage von Kriteriumswerten, "für deren Ermittlung die Schätzung der sogenannten Regressionskoeffizienten - Steigungen und yAchsenabschnitt - notwendig ist" (Bortz und Schuster 2010, S. 342). Der Regressionskoeffizient oder Steigungskoeffizient eines Prädiktors beschreibt den Einfluss des Prädiktors auf das Kriterium (abhängige Variable). Im nächsten Schritt wird geprüft, ob und in welchem Ausmaß sich weitere Prädiktorvariablen auf den Steigungskoeffizienten und das 
Regressionsmodell auswirken. Durch das nacheinander schrittweise Hinzunehmen (Vorwärtsselektion) und Entfernen (Rückwärtselimination) von weiteren Prädiktorvariablen können genau diejenigen Faktoren identifiziert werden, die einen Einfluss auf die Regressionsgleichung nehmen. Durch die Regressionskoeffizienten wird dieser Einfluss quantifiziert (Bortz und Schuster 2010; Leonhart 2004).

\subsection{Bildung der Odds Ratio}

Aus den Regressionskoeffizienten werden nachfolgend die sogenannten „Odds Ratios" gebildet. Sie stellen eine Möglichkeit zur Ergebnisinterpretation der logistischen Regressionsanalyse dar. Mit der Odds Ratio wird das bedingte Wahrscheinlichkeitsverhältnis in Form eines Faktors für das Eintreten eines Ereignisses in Abhängigkeit von einer anderen Variablen ausgedrückt.

Der Zusammenhang zwischen den unabhängigen Variablen und den über die logistische Funktion bestimmten Wahrscheinlichkeiten ist nicht linear. Daraus ergibt sich ein unmittelbares Interpretationsproblem. Da die unabhängigen Variablen den Exponenten der e-Funktion bestimmen, beeinflussen sie einerseits nur indirekt über die Wahrscheinlichkeitsberechnung und andererseits in nicht-linearer Form (durch die untergestellte logistische Funktion) die Eintrittswahrscheinlichkeit für das Ereignis. „Das hat zur Konsequenz, dass weder die Regressionskoeffizienten untereinander vergleichbar sind, noch dass die Wirkung der unabhängigen Variablen“ (Backhaus et al. 2008, S. 256) innerhalb ihrer Ausprägung bis in die Randbereiche konstant beziehungsweise linear ist. Eine lineare Interpretation, das heißt eine genaue Aussage über die Höhe der Einflussstärke der Variablen auf die Eintrittswahrscheinlichkeit, kann nur dadurch erfolgen, dass nicht „die Eintrittswahrscheinlichkeit $P(Y=1)$, sondern ihr Verhältnis zur Gegenwahrscheinlichkeit $P(Y=0)$ beziehungsweise 1-P(Y=1) betrachtet wird“ (Backhaus et al. 2008, S. 258). Dieses Wahrscheinlichkeitsverhältnis stellt die Chance (Odd) dar, das Ereignis $\mathrm{Y}=1$ im Vergleich zum Ereignis $\mathrm{Y}=0$ zu erhalten. Die Odds entwickeln sich entsprechend der e-Funktion mit dem Wert des jeweiligen Regressionskoeffizienten als Exponenten.

Die Odds Ratio gibt nun denjenigen Faktor an, um den die Chance für das Eintreten des Ereignisses steigt oder fällt, wenn der dazugehörige Risikofaktor vorhanden ist. Bei kategorischen unabhängigen Variablen ist die Odds Ratio der Faktor, um den die Chance für das Eintreten eines Ereignisses steigt oder fällt. Erhöht sich eine kontinuierliche unab- 
hängige Variable um eine Einheit, „so vergrößert sich das Chancenverhältnis zu Gunsten des Ereignisses" (Backhaus et al. 2008, S. 259) um den Faktor e potenziert mit dem Regressionskoeffizienten. Die Odds Ratio kann Werte zwischen 0 bis $\infty$ annehmen, wobei eine Odds Ratio = 1 bedeutet, dass die Variable keinen Einfluss auf das Ereignis hat, bei einer Odds Ratio $<1$ reduziert sich die Chance des Eintretens des Ereignisses und eine Odds Ratio $>1$ begünstigt das Eintreten des Ereignisses (Backhaus et al. 2008). Grundsätzlich sollten für alle Odds Ratios die entsprechenden 95\%-Konfidenzintervalle angegeben werden. Darüber hinaus ist zu beachten, dass nur dann eine Aussage über den Einfluss einer unabhängigen Variablen getroffen werden kann, wenn der Wertebereich des 95\%Konfidenzintervalls der zugehörigen Odds Ratio entweder nur bei Werten $>1$ oder nur $<1$ liegt. Intervalle, die die Zahl 1 enthalten (beispielsweise 0,5 - 1,5), besitzen keinen eindeutigen Einfluss.

\subsection{Bildung der Score-Punkte}

Im Rahmen dieser Studie wurden zwei logistische Regressionsanalysen durchgeführt, die anfangs nur die präoperativen Faktoren und darauffolgend die prä- und intraoperativen Variablen berücksichtigten. Hierzu wurden zunächst alle kontinuierlichen Parameter (wie Alter oder Kreatinin) dichotomisiert, das heißt in nominalskalierte oder kategoriale Form „vorhanden“ und „nicht vorhanden“ überführt. Anschließend wurde mittels logistischer Regression der Einfluss der einzelnen nominalskalierten unabhängigen Variablen auf das Auftreten einer akuten Nierenschädigung - der abhängigen Variable oder Kriteriumsvariable - geprüft. Es wurde eine Regressionsfunktion gebildet und die einzelnen Regressionskoeffizienten wurden berechnet. Diese beschreiben den Effekt der einzelnen Parameter auf die Entstehung einer akuten Nierenschädigung, was man auch als relatives Risiko der einzelnen Variablen bezeichnen kann.

Anschließend wurden aus den Regressionskoeffizienten die Punktwerte für die einzelnen Risikofaktoren hergeleitet - die Scorepunkte. Die Berechnung erfolgte analog zum Vorgehen von Thakar. Infolgedessen wurden alle Regressionskoeffizienten der berücksichtigten Einflussparameter mit dem Kehrwert des kleinsten Regressionskoeffizienten multipliziert (Thakar et al. 2005; Toll et al. 2008). Dadurch erhält der kleinste Risikofaktor immer eine Scoregewichtung von 1 (Division durch seinen eigenen Wert) und die Basis eines Scores ist ebenso immer der Wert 1, wodurch einerseits eine Vergleichbarkeit der Einflussgrößen und andererseits eine Vergleichbarkeit von verschiedenen Scores unterei- 
nander gewährleistet ist. Zur einfacheren Anwendung wird der multiplizierte Wert auf den nächstgelegenen ganzzahligen Wert gerundet, wobei Thakar bereits den Kehrwert des kleinsten Koeffizienten rundete und mit diesem multiplizierte (Thakar et al. 2005).

Durch dieses Verfahren kann eine risikoadaptierte Gewichtung der einzelnen Risikofaktoren erreicht werden. Die Addition der einzelnen Scorepunkte ergibt schließlich den Gesamtsummenscore. Es wird der Minimalwert 0 bei keinem Risikofaktor und der Maximalwert des Gesamtscores bei Vorhandensein aller Risikofaktoren vergeben. Die Höhe des Summenscorewertes gibt nun das individuelle Risiko an, eine akute Nierenschädigung zu erleiden. Eine Aufteilung in Risikokategorien anhand der Punkte wird vorgenommen.

In der vorliegenden Arbeit wurden auf diese Weise zwei modifizierte Scores entwickelt:

- Score 1 bestehend aus präoperativen Risikofaktoren

- Score 2 bestehend aus prä- und intraoperativen Risikofaktoren.

\subsection{Validierung der modifizierten Scores}

Im letzten Schritt wurden die modifizierten Scores validiert. Das Patientenkollektiv für die Validierung bestand aus dem Evaluierungskollektiv. Hierzu wurde analog zur Validierung des Original-Scores (siehe Kapitel 2.6.2.2, S. 43) die Diskriminationsfähigkeit beurteilt, indem in der ROC-Analyse die Fläche unter der Kurve berechnet wurde. Es erfolgten ein Vergleich mit den Ergebnissen des Thakar-Scores und eine Beurteilung, ob die Modifizierungen und Erweiterungen des Original-Scores eine Verbesserung hinsichtlich der prognostischen Aussagekraft erzielen konnten.

\subsubsection{Einfluss der Natriumbicarbonat-Therapie auf die postoperative Nierenfunktion}

Innerhalb der vorliegenden Studie wurde der Einfluss der Gabe von Natriumbicarbonat auf die AKI-Inzidenz an 448 Patienten untersucht. Die Patienten wurden in eine $\mathrm{NaHCO}_{3}$-Gruppe, die eine Therapie mit Natriumbicarbonat erhielt und in eine Kontrollgruppe, die kein Natriumbicarbonat erhielt, unterteilt. 
Zunächst musste die Vergleichbarkeit des Risikos eines AKI der beiden Kollektive sichergestellt werden. Zu diesem Zweck erfolgte ein Vergleich der beiden Patientenkollektive einerseits hinsichtlich der patientenspezifischen Risikofaktoren durch Erhebung der individuellen Scorepunkte nach Thakar und andererseits hinsichtlich des HLM-abhängigen Hämolyseausmaßes durch Erhebung der intraoperativen freien Serumhämoglobinwerte. Hierzu dienten die während der extrakorporalen Zirkulation zu mehrfachen Messzeitpunkten bestimmten freien Serumhämoglobinkonzentrationen. Der jeweilige Mittelwert aller individuellen Messwerte wurde berücksichtigt.

Der Test auf Normalverteilung erfolgte mittels Kolmogorow-Smirnow-Test. Mit dem Mann-Whitney-U-Test wurde anschließend überprüft, ob sich die nicht-normalverteilte kontinuierliche Variable „freie Hämoglobinkonzentration“ innerhalb der $\mathrm{NaHCO}_{3}$-Gruppe signifikant von derjenigen ohne Natriumbicarbonattherapie unterscheidet. Mittels MannWhitney-U-Test wurde auch die Verteilung der nicht-normalverteilten Scorepunkte zwischen $\mathrm{NaHCO}_{3}-\mathrm{Gruppe}$ und Kontrollgruppe überprüft.

Zur Untersuchung des Effekts der Natriumbicarbonattherapie auf das Auftreten einer akuten Nierenschädigung wurden die Inzidenzen eines AKI gemäß der AKINKlassifikation in der $\mathrm{NaHCO}_{3}$-Gruppe und der Kontrollgruppe mittels einer zweidimensionalen Kontingenztabelle beziehungsweise Vierfeldertafel verglichen.

Anschließend wurde mit Hilfe des $\mathrm{Chi}^{2}$-Tests bestimmt, ob in der Häufigkeitsverteilung der Merkmale Natriumbicarbonatgabe und AKI ein signifikanter Unterschied besteht. 


\section{ERGEBNISSE}

\subsection{Demographie des Patientenkollektivs}

In die prospektive Beobachtungsstudie wurden 1.007 Patienten der operativen Intensivstation 0118 des Universitätsklinikums Göttingen von Juli 2009 bis Februar 2011 eingeschlossen.

748 männliche Patienten und 259 Patienten weiblichen Geschlechts wurden unter Einsatz eines kardiopulmonalen Bypasses operiert. Das mittlere Alter der Patienten betrug 68 Jahre. Der älteste Patient war 88 Jahre alt, der jüngste 21. Der Interquartil-Bereich lag zwischen 74,75 und 62 Jahren.

\subsection{Häufigkeitsverteilung der untersuchten Risikofaktoren}

Es wurden 171 (16,98 \%) sonstige Operationen, 166 (16,48\%) Herzklappenoperationen („Klappe“) und 133 (13,21\%) Aortokoronare Bypass-Operationen mit zusätzlich kombiniertem Ersatz beziehungsweise Rekonstruktion der Herzklappen („ACB+Klappe“) durchgeführt. Sonstige Operationen umfassten im Göttinger Kollektiv folgende Eingriffe: Myektomien (bei hypertroph-obstruktiven Kardiomyopathien), Papillarmuskelrekonstruktionen, Herzohrverschlüsse, ASD-Verschlüsse, Operationen am Aortenbogen, AneurysmaOperationen; oben genannte Eingriffe und Aortokoronare Bypass-Operationen in Kombination mit Ablationsverfahren, Tumorchirurgie, Lungenoperationen, Thymektomien oder Eingriffen an den Karotisgefäßen. Bei 91 Patienten handelte es sich um einen NotfallEingriff, bei 53 um einen Wiederholungseingriff (Re-Operation).

Anamnestisch konnten folgende Ergebnisse gezeigt werden: Bei 420 (41,71 \%) Patienten konnte eine Herzinsuffizienz (NYHA III und NYHA IV) festgestellt werden. Ein erhöhter präoperativer Kreatininwert (1,2 - 2,0 mg/dl) zeigte sich bei 202 (20,06 \%) Patienten. $148(14,7 \%)$ Patienten litten unter einer COPD, 140 (13,9 \%) unter einem insulinpflichtigen Diabetes mellitus. 119 (11,82 \%) Patienten besaßen eine linksventrikuläre Ejektionsfraktion < $35 \%, 19(1,89 \%)$ hatten eine präoperative IABP und 17 (1,69\%) Patienten einen massiv erhöhten Kreatininwert ( $\geq 2,1 \mathrm{mg} / \mathrm{dl})$. 
Tabelle 3.1 Verteilung der Risikofaktoren in abnehmender Häufigkeit

$\mathrm{CHF}=$ chronische Herzinsuffizienz, $\mathrm{Krea}=$ Serumkreatinin in $\mathrm{mg} / \mathrm{dl}, \mathrm{IDDM}=$ Insulinpflichtiger Diabetes mellitus, LVEF = Linksventrikuläre Ejektionsfraktion, IABP = Intraaortale Ballonpumpe.

\begin{tabular}{lcc}
\hline Risikofaktor & $\mathrm{N}$ & $\%$ \\
\hline CHF & 420 & 41,71 \\
Weiblich & 259 & 25,72 \\
Krea $1,2-2,0$ & 202 & 20,06 \\
Sonstige OP & 171 & 16,98 \\
Klappe & 166 & 16,48 \\
COPD & 148 & 14,70 \\
IDDM & 140 & 13,90 \\
ACB + Klappe & 133 & 13,21 \\
LVEF $<35$ & 119 & 11,82 \\
Not-OP & 91 & 9,04 \\
Re-OP & 53 & 5,26 \\
IABP & 19 & 1,89 \\
Krea $\geq 2,1$ & 17 & 1,69 \\
\hline
\end{tabular}

In Kapitel 3.6.1 folgen die Ergebnisse der univariaten Analyse mit Signifikanzprüfung der einzelnen Risikofaktoren.

Die intraoperativen Risikofaktoren stellten sich wie folgt dar: die mittlere Dauer der extrakorporalen Zirkulation betrug 131 Minuten, im Bereich zwischen 150 und 95 Minuten lagen 50 \% der Werte. Die Transfusion von Erythrozytenkonzentraten (EK) erfolgte bei 469 Patienten (46,6 \%), FFP bei 38 Patienten (3,8 \%) und TK bei 92 Patienten (9,1\%). Aufgrund dieser niedrigen Transfusionsrate betrugen Median und IQR für die Anzahl gegebener Blutprodukte für die Kategorien FFP und TK 0 beziehungsweise 0 - 0 und für die Kategorie EK 0 beziehungsweise 2 - 0 . Eine Filtration über die Herz-Lungen-Maschine erfolgte in 27 Fällen mit durchschnittlich 2915 ml. 


\subsection{Häufigkeitsverteilung der Scorewerte}

Anhand der präoperativen Risikofaktoren wurde für jeden Patienten der individuelle Scorewert nach Thakar errechnet, wobei der Minimalwert der erreichten Scorewerte bei 0 lag und der Maximalwert bei 14 von 17 möglichen Punkten. Der Median lag bei 2 Punkten (IQR 1 - 4).

Der häufigste Scorewert war 2 mit 21,65\%, gefolgt von Wert $3(18,87 \%)$ und Wert 0 (16,78\%). Ab dem Scorewert 3 war die Häufigkeit abnehmend. 95 \% der Patienten erhielten Werte $\leq 6$. Die Häufigkeit der Werte 7-11 und 14 lag insgesamt bei nur 3,77\% und fiel mit steigender Scorezahl. Die Scores 12, 13, 15, 16 und 17 wurden nicht vergeben.

Tabelle 3.2 Häufigkeitsverteilung der Scorewerte (in allen AKIN-Gruppen)

\begin{tabular}{ccc} 
Scorepunkte & $\mathrm{N}$ & $\%$ \\
\hline 0 & 169 & 16,78 \\
1 & 141 & 14,00 \\
2 & 218 & 21,65 \\
3 & 190 & 18,87 \\
4 & 126 & 12,52 \\
5 & 78 & 7,75 \\
6 & 47 & 4,67 \\
7 & 18 & 1,79 \\
8 & 8 & 0,80 \\
9 & 5 & 0,50 \\
10 & 4 & 0,40 \\
11 & 2 & 0,20 \\
14 & 1 & 0,10 \\
\hline
\end{tabular}




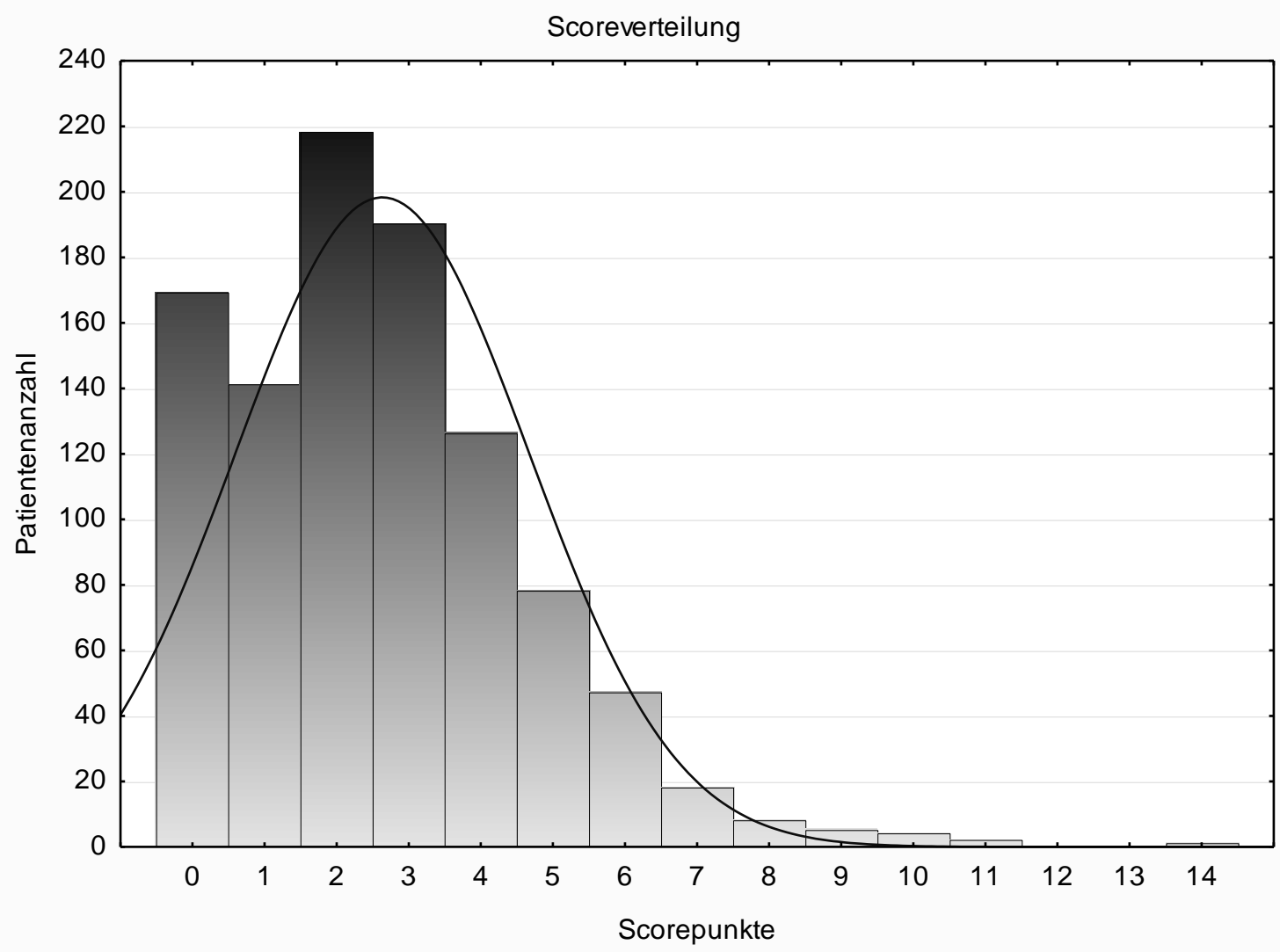

Abbildung 3.1 Histogramm der Scoreverteilung 


\subsection{Inzidenz des AKI im Göttinger Kollektiv}

\subsubsection{Häufigkeitsverteilung der AKIN-Stadien}

$484(48,06 \%)$ von 1.007 Patienten hatten keine akute Nierenschädigung (AKIN 0). $523(51,94 \%)$ erlitten dagegen eine akute Nierenschädigung, wovon 321 nur leichte Diureseeinschränkungen und Kreatininwertanstiege verzeichneten (AKIN 1), 88 Patienten dem Stadium AKIN 2 zugeordnet werden konnten und 114 Patienten das Stadium AKIN 3 erreichten.

Tabelle 3.3 Häufigkeitsverteilung der AKIN-Stadien

\begin{tabular}{ccc} 
AKIN-Stadium & N & $\%$ \\
\hline 0 & 484 & 48,06 \\
1 & 321 & 31,88 \\
2 & 88 & 8,74 \\
3 & 114 & 11,32 \\
\hline
\end{tabular}

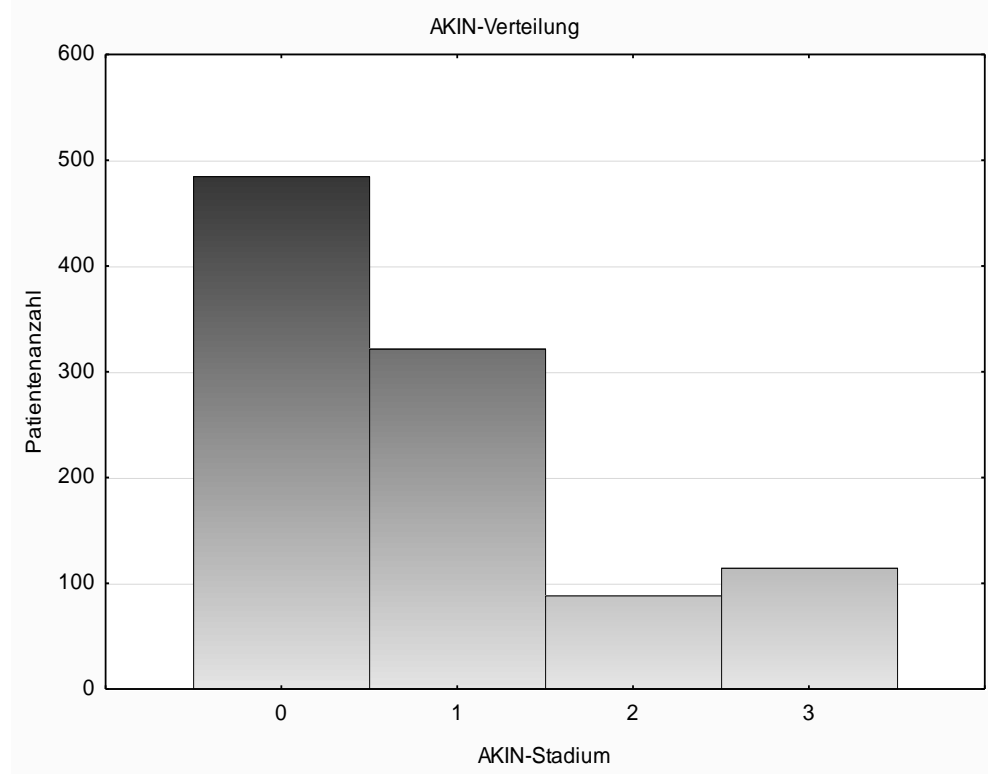

Abbildung 3.2 Histogramm der AKIN-Inzidenzen

3.4.2 Inzidenzen der akuten Nierenschädigung in Abhängigkeit der Scorepunkte

Etwas mehr als die Hälfte der Patienten im Untersuchungskollektiv erlitten eine postoperative akute Nierenschädigung: 523 (51,94\%) Patienten. Die Inzidenz des AKI er- 
höhte sich mit steigendem Scorewert. Doch auch Patienten ohne Risikofaktoren nach Thakar (Scorewert 0) wiesen in 37\% eine Nierenschädigung auf.

Tabelle 3.4 Inzidenz AKIN 1-3

\begin{tabular}{ccc}
\hline Scorepunkte & \multicolumn{2}{c}{ AKIN 1-3 } \\
& N & $\%$ \\
\hline 0 & 62 & 36,69 \\
1 & 64 & 45,39 \\
2 & 94 & 43,12 \\
3 & 102 & 53,68 \\
4 & 82 & 65,08 \\
5 & 55 & 70,51 \\
6 & 33 & 70,21 \\
7 & 14 & 77,78 \\
8 & 5 & 62,5 \\
9 & 5 & 100 \\
10 & 4 & 100 \\
11 & 2 & 100 \\
12 & 0 & 0 \\
13 & 0 & 0 \\
14 & 1 & 100 \\
\hline
\end{tabular}

AKI-Häufigkeit in Abhängigkeit der Scorewerte

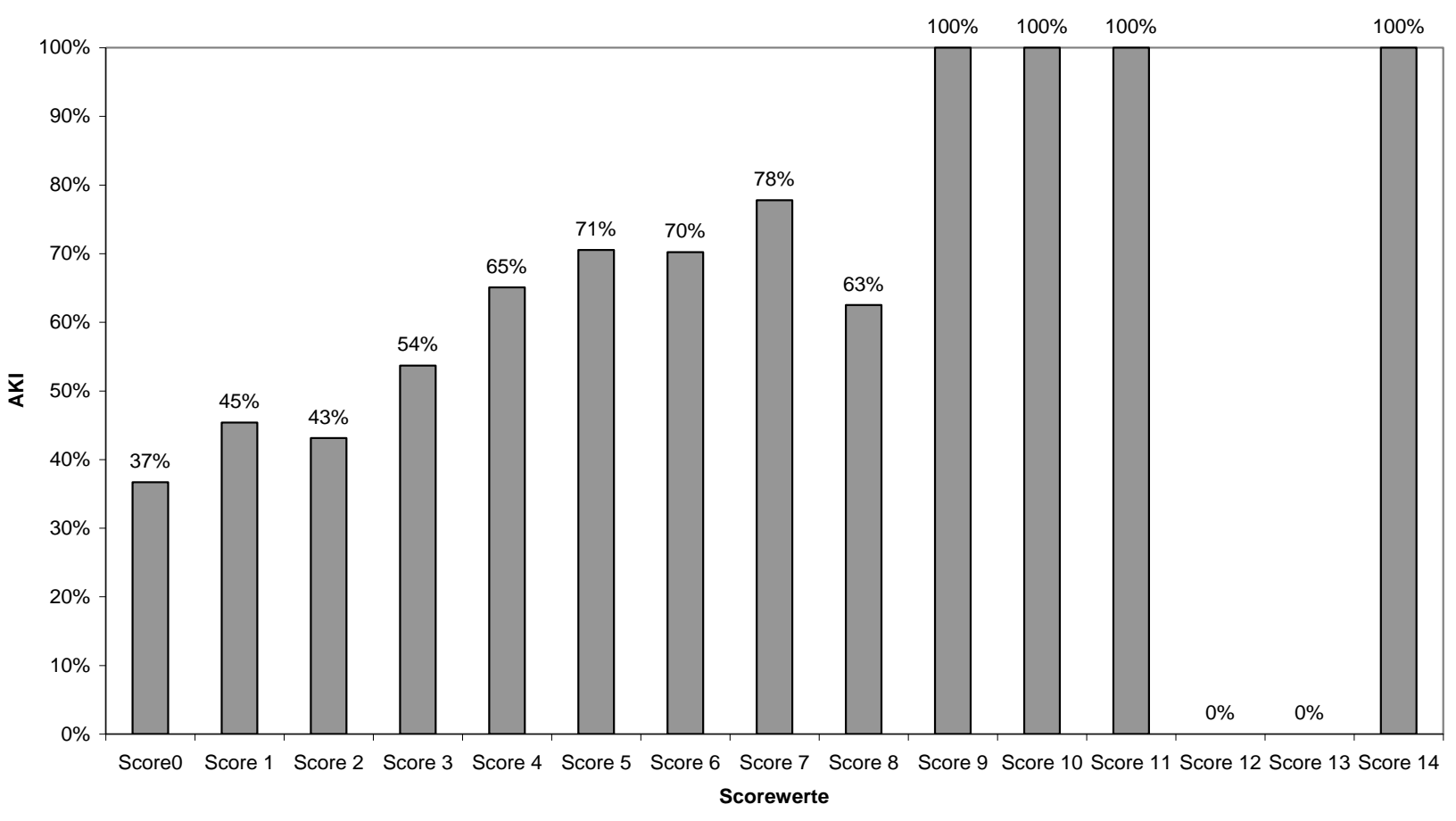

Abbildung 3.3 AKI in Abhängigkeit der Scorewerte 


\subsubsection{Score-Kategorien}

Analog zum Verfahren nach Thakar wurden die Patienten anhand ihrer individuellen Scorewerte zu vier Scorekategorien bzw. Risikokategorien zugeordnet. Es konnte gezeigt werden, dass die Inzidenz der akuten Nierenschädigung (AKIN 1-3) mit zunehmender Scorekategorie ansteigt.

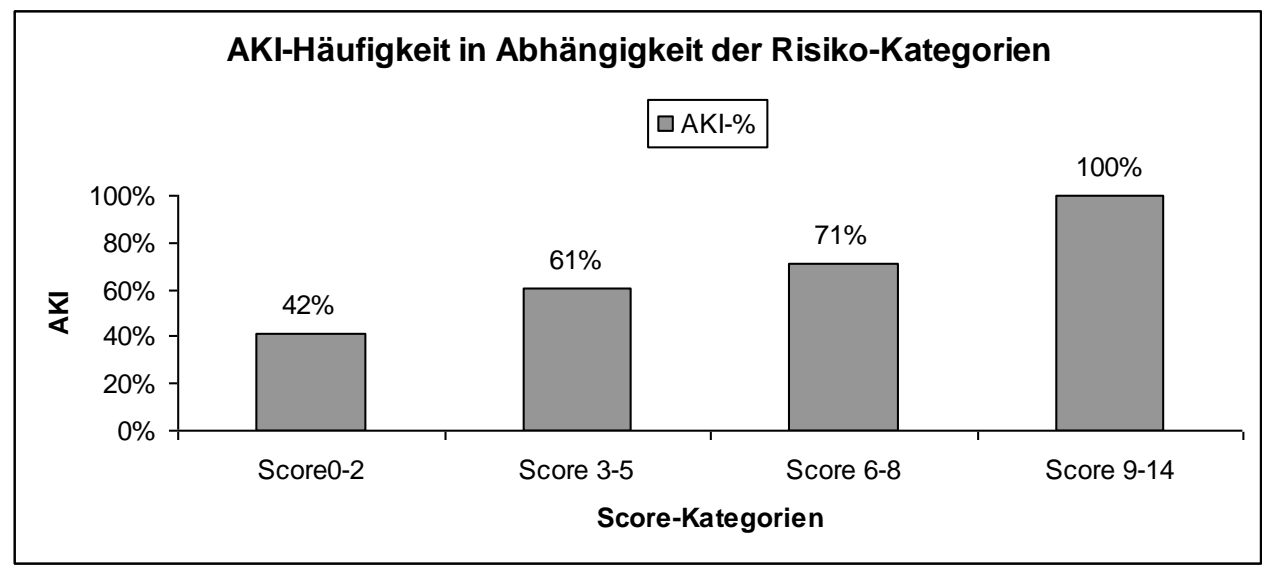

Abbildung 3.4 AKI in Abhängigkeit der Risiko-Kategorien

\subsection{Validierung des Thakar-Scores}

\subsubsection{Kalibrierung des Thakar-Scores bei Anwendung am Göttinger Kollektiv}

Aus dem Vergleich der Göttinger Inzidenzen eines AKI (unabhängig der AKIN-Klasse) in jeder Scoregruppe mit dem Cleveland-Kollektiv geht hervor, dass die tatsächlichen Inzidenzen die von Thakar prognostizierten Inzidenzen weit verfehlen. Keine Göttinger Inzidenz liegt im Bereich des prognostizierten Konfidenzintervalls. 
Tabelle 3.5 AKI-Inzidenzen im Göttinger Kollektiv im Vergleich zu Cleveland

\begin{tabular}{cccc}
\hline Scorepunkte & AKIN 1-3 & THAKAR & THAKAR \\
& $\%$ & $\%$ & $95 \% \mathrm{KI}$ \\
\hline 0 & 36,69 & 0,05 & $0-0,26$ \\
1 & 45,39 & 0,29 & $0,13-0,57$ \\
2 & 43,12 & 0,71 & $0,46-1,04$ \\
3 & 53,68 & 1,19 & $0,83-1,65$ \\
4 & 65,08 & 2,06 & $1,47-8,53$ \\
5 & 70,51 & 3,04 & $2,16-4,14$ \\
6 & 70,21 & 6,42 & $4,71-8,53$ \\
7 & 77,78 & 9,6 & $6,67-13,15$ \\
8 & 62,5 & 9,83 & $5,83-15,27$ \\
9 & 100 & 21,95 & $13,56-32,46$ \\
10 & 100 & 12,5 & $4,19-26,8$ \\
11 & 100 & 21,43 & $4,66-50,08$ \\
12 & 0 & 57,13 & $18,41-90,1$ \\
13 & 0 & 100 & $2,5-100$ \\
14 & 100 & - & - \\
\hline
\end{tabular}

\subsubsection{Diskrimination des Thakar-Scores bei Anwendung am Göttinger Kollektiv}

Zur Beurteilung der Diskriminationsfähigkeit des Thakar-Scores wurde eine ROCAnalyse durchgeführt. Der optimale Cut-off-Point wurde als $>2$ Scorepunkte ermittelt. Hierbei ergaben sich eine maximale Sensitivität von 57,74 \% und eine maximale Spezifität von $64,26 \%$. Die Fläche unter der Kurve (AUC) wurde mit einem Wert von 0,638 berechnet. Weniger als 2 Scorepunkte gingen mit einer größeren Sensitivität und fallender Spezifität einher, bei mehr als 3 Scorepunkten verhielt es sich umgekehrt. Das $95 \%$ Konfidenzintervall der AUC war 0,608-0,668, damit war die AUC signifikant geringer als bei Thakar (AUC 0,81 im Evaluierungskollektiv und 0,82 im Validierungskollektiv). 


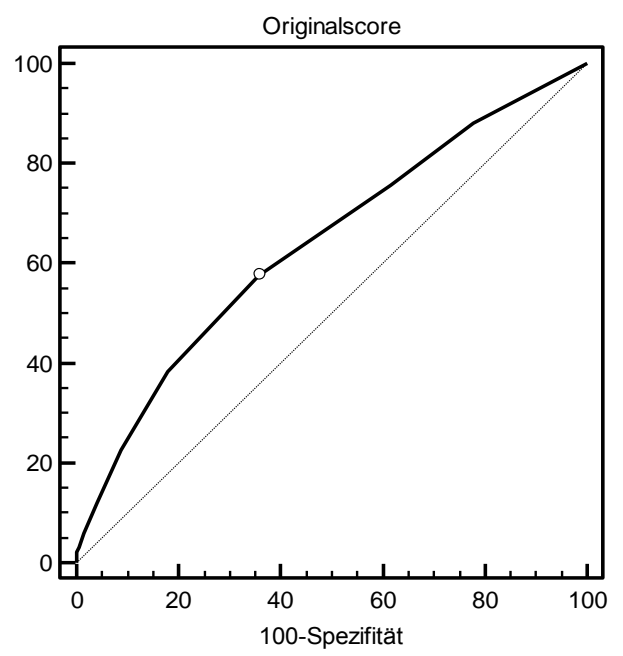

Abbildung 3.5 ROC-Kurve des Original-Scores

\subsection{Re-Evaluierung des Thakar-Scores am Göttinger Kollektiv}

\subsubsection{Univariate Analyse der Risikofaktoren}

Mit Hilfe von Kontingenztabellen wurden zunächst alle Risikofaktoren des OriginalScores hinsichtlich ihrer Verteilung im Patientenkollektiv mit einer akuten Nierenschädigung (AKIN 1-3) und der Gruppe ohne akute Nierenschädigung (AKIN 0) untersucht. Die Risikofaktoren Herzinsuffizienz, Kreatininwert zwischen 1,2 mg/dl und 2,0 mg/dl, insulinpflichtiger Diabetes mellitus und linksventrikuläre Ejektionsfraktion < $35 \%$ zeigten einen hoch signifikanten Unterschied $(p<0,001)$. Die Art des Eingriffs war signifikant unterschiedlich verteilt $(p<0,05)$.

Für folgende Original-Risikofaktoren konnte keine signifikant unterschiedliche Verteilung nachgewiesen werden: Serumkreatinin $\geq 2,1 \mathrm{mg} / \mathrm{dl}$, Not-Operation, präoperative Anlage einer IABP, Re-Operation, COPD sowie weibliches Geschlecht. 
Tabelle 3.6 Univariate Analyse der Risikofaktoren

\begin{tabular}{|c|c|c|c|}
\hline Risikofaktor $(\mathrm{N}=1007)$ & AKI 1-3 $(\mathrm{N}=523)$ & AKI $0(N=484)$ & P-Wert \\
\hline CHF & & & $P<0,0001$ \\
\hline ja $(\mathrm{N}=420)$ & 253 & 167 & \\
\hline nein $(N=587)$ & 270 & 317 & \\
\hline Krea $1,2-2,0$ & & & $P<0,0001$ \\
\hline ja $(\mathrm{N}=202)$ & 146 & 56 & \\
\hline nein $(N=805)$ & 377 & 428 & \\
\hline IDDM & & & $P=0,0002$ \\
\hline ja $(\mathrm{N}=140)$ & 94 & 46 & \\
\hline nein $(N=867)$ & 429 & 438 & \\
\hline EF & & & $P=0,0005$ \\
\hline$<35(\mathrm{~N}=119)$ & 80 & 39 & \\
\hline$>35(\mathrm{~N}=888)$ & 443 & 445 & \\
\hline Art des Eingriffs & & & $P=0,0062$ \\
\hline ACB-OP $(N=537)$ & 259 & 278 & \\
\hline Klappen-OP (N=166) & 83 & 83 & \\
\hline ACB- Klappen-OP ( $\mathrm{N}=133)$ & 85 & 48 & \\
\hline Sonstige OP (N=171) & 96 & 75 & \\
\hline Krea $\geq 2,1$ & & & $P=0,0723$ \\
\hline ja $(\mathrm{N}=17)$ & 13 & 4 & \\
\hline nein $(N=990)$ & 510 & 480 & \\
\hline Not-OP & & & $P=0,1700$ \\
\hline $\mathrm{ja}(\mathrm{N}=91)$ & 54 & 37 & \\
\hline nein $(N=916)$ & 469 & 447 & \\
\hline IABP & & & $P=0,2224$ \\
\hline ja $(\mathrm{N}=19)$ & 13 & 6 & \\
\hline nein $(N=988)$ & 510 & 478 & \\
\hline Re-OP & & & $P=0,2617$ \\
\hline $\mathrm{ja}(\mathrm{N}=53)$ & 32 & 21 & \\
\hline nein $(N=954)$ & 491 & 463 & \\
\hline COPD & & & $P=0,5174$ \\
\hline ja $(\mathrm{N}=148)$ & 81 & 67 & \\
\hline nein $(N=859)$ & 442 & 417 & \\
\hline Geschlecht & & & $P=0,6667$ \\
\hline weiblich ( $N=259$ ) & 138 & 121 & \\
\hline männlich ( $N=748)$ & 385 & 363 & \\
\hline
\end{tabular}




\subsubsection{Multivariate Analyse}

Nach Identifizierung der signifikant unterschiedlich verteilten Parameter mittels univariater Analyse konnte mit Hilfe der logistischen Regression für alle in Tabelle 3.6 (S. 63) aufgelisteten signifikanten Parameter auch ein unabhängiger Einfluss auf die Entstehung eines AKI nachgewiesen werden. Innerhalb des Parameters „Art des Eingriffes“ konnte sowohl für die Variable „sonstige Operation“ als auch für den „kombinierten ACBHerzklappeneingriff“ ein unabhängiger Einfluss gezeigt werden; wobei eine „alleinige Herzklappenoperation" keinen unabhängigen Einfluss nahm. Unten stehende Tabelle zeigt die errechneten Regressionskoeffizienten, Odds Ratios mit dem jeweiligen $95 \%$ Konfidenzintervall und die daraus ermittelten Scorepunkte für die fünf Einflussnehmenden Risikofaktoren. Die einzelnen Scorepunkte lagen zwischen einem und drei Punkten, mit einem Gesamtsummen-Minimum von 0 und einem Maximum von 9 Scorepunkten.

Tabelle 3.7 Regressionskoeffizienten, Odds Ratios und Scorepunkte

\begin{tabular}{lcccc}
\hline Risikofaktor & $\begin{array}{c}\text { Regressions- } \\
\text { koeffizient }\end{array}$ & Odds Ratio & $95 \% \mathrm{KI}$ & $\begin{array}{c}\text { Score- } \\
\text { punkte }\end{array}$ \\
\hline CHF & 0,37 & 1,45 & $1,11-1,91$ & 1 \\
EF $<35$ & 0,50 & 1,65 & $1,07-2,53$ & 1 \\
IDDM & 0,58 & 1,79 & $1,21-2,66$ & 2 \\
Krea 1,2-2,0 & 0,98 & 2,67 & $1,89-3,78$ & 3 \\
Art des Eingriffs & & & & \\
\multicolumn{1}{c}{ sonstige OP } & 0,39 & 1,47 & $1,04-2,09$ & 1 \\
\multicolumn{1}{c}{ ACB-Klappen-OP } & 0,61 & 1,83 & $1,23-2,73$ & 2 \\
\hline
\end{tabular}

Die $95 \%$ - Konfidenzintervalle der einzelnen Odds Ratios lagen alle im Bereich > 1, sodass ein eindeutiger Einfluss festzustellen war.

\subsubsection{ROC-Analyse des modifizierten Scores}

Die Diskriminationsfähigkeit verbesserte sich durch diese Modifikation des OriginalScores geringfügig, die AUC in der ROC-Analyse stieg von 0,638 auf 0,652 an, was jedoch 
nicht statistisch signifikant war $(p=0,53)$. Der optimale Cut-Off-Point wurde auf $>1$ Scorepunkt festgelegt, mit einer Sensitivität von 57,93 \% und einer Spezifität von 64,88 \%.

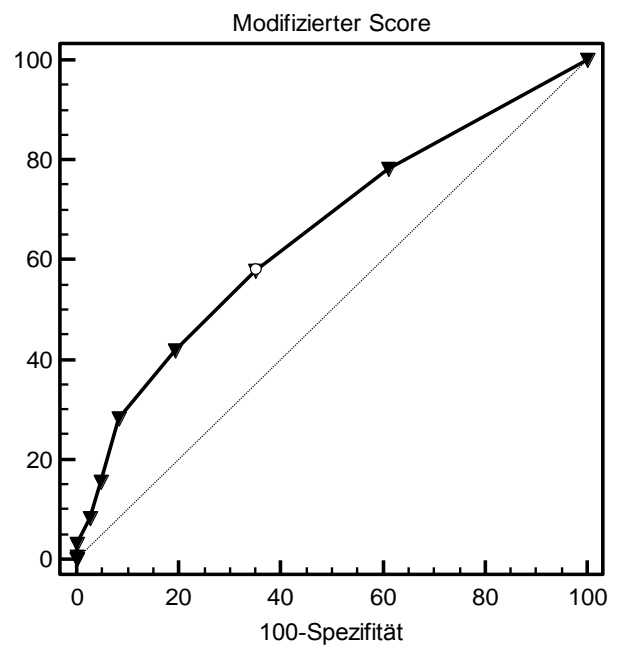

Abbildung 3.6 ROC-Kurve des modifizierten Scores

\subsection{Erweiterung des Scores}

\subsubsection{Univariate Analyse der kontinuierlichen Risikofaktoren}

Zunächst wurde mittels univariater Analyse die Verteilung der potentiellen Risikofaktoren zwischen den Gruppen AKIN 0 und AKIN > 0 untersucht. Es zeigte sich eine signifikant unterschiedliche Verteilung aller Risikofaktoren $(p<0,01)$, außer für die kategorische Variable Filtration.

Tabelle 3.8 Verteilung der potentiellen Risikofaktoren

\begin{tabular}{lccc}
\hline kontinuierliche Variablen & $\begin{array}{c}\text { AKI 1-3 } \\
\text { (Median) }\end{array}$ & $\begin{array}{c}\text { AKI 0 } \\
\text { (Median) }\end{array}$ & P-Wert \\
\hline Alter & 72 & 67 & $\mathbf{P}<\mathbf{0 , 0 0 0 1}$ \\
HLM-Zeit & 130 & 112 & $\mathbf{P}<\mathbf{0 , 0 0 0 1}$ \\
Aortenklemmzeit & 78 & 70 & $\mathbf{P}<\mathbf{0 , 0 0 0 1}$ \\
EK-Anzahl & 2 & 0 & $\mathbf{P}<\mathbf{0 , 0 0 0 1}$ \\
FFP-Anzahl & 0 & 0 & $\mathbf{P}=\mathbf{0 , 0 0 0 1}$ \\
TK-Anzahl & 0 & 0 & $\mathbf{P}=\mathbf{0 , 0 0 1 8}$ \\
& & & \\
\hline & AKI 1-3 & AKI 0 & \\
kategorische Variable & $(\mathrm{N})$ & (N) & \\
\hline Filtration & 12 & 15 & \\
keine Filtration & 511 & 469 & $\mathrm{P}=0,5521$ \\
\hline
\end{tabular}




\subsubsection{Dichotomisierung der kontinuierlichen Risikofaktoren}

Zur Dichotomisierung der kontinuierlichen Risikofaktoren „EK-Anzahl“, „FFPAnzahl“, „TK-Anzahl“, „HLM-Zeit“, „Aortenklemmzeit" und „Alter" wurde mittels ROCAnalysen die jeweiligen Cut-Off-Werte bestimmt. Folgende Cut-Off-Werte wurden festgelegt:

Tabelle 3.9 Kontinuierliche Variablen

\begin{tabular}{lc}
\hline Variable & Cut-Off-Point \\
\hline EK-Anzahl & 1 \\
FFP-Anzahl & 0 \\
TK-Anzahl & 0 \\
HLM-Zeit & 134 \\
Aortenklemmzeit & 77 \\
Alter & 68 \\
\hline
\end{tabular}

\subsubsection{Multivariate Analyse}

In der logistischen Regressionsanalyse mit den prä- und intraoperativen Risikofaktoren konnte für fünf weitere Risikofaktoren ein Einfluss auf die Entstehung der akuten Nierenschädigung gezeigt werden: männliches Geschlecht, Alter $>68$ Jahre, $>1$ Erythrozytenkonzentrat, >0 FFP und HLM-Zeit > 134 Minuten. Aus dem Original-Score konnte nur für die vier Risikofaktoren Geschlecht, chronische Herzinsuffizienz, insulinpflichtiger Diabetes mellitus und ein Kreatininwert zwischen $1,2 \mathrm{mg} / \mathrm{dl}$ und 2,0 mg/dl ein unabhängiger Einfluss nachgewiesen werden. Für die übrigen Original-Risikofaktoren und intraoperativen Variablen Aortenklemmzeit und Thrombozytenkonzentrat-Gabe konnte kein unabhängiger Einfluss gezeigt werden.

\subsubsection{Scorepunkte der Risikofaktoren}

Der erweiterte Score besteht dementsprechend aus fünf präoperativen Risikofaktoren und drei intraoperativen Risikofaktoren, deren jeweilige Scorewerte zwischen einem und drei Punkten variieren. Die minimale Scoresumme beträgt 0 Punkte, bei Präsenz aller Risikofaktoren werden 14 Punkte vergeben. 
Tabelle 3.10 Regressionskoeffizienten, Odds Ratios und Scorepunkte des erweiterten Scores

\begin{tabular}{lcccc}
\hline Risikofaktor & $\begin{array}{c}\text { Regressions- } \\
\text { koeffizient }\end{array}$ & $\begin{array}{c}\text { Odds } \\
\text { Ratio }\end{array}$ & $95 \% \mathrm{KI}$ & $\begin{array}{c}\text { Score- } \\
\text { punkte }\end{array}$ \\
\hline präoperativ & & & & \\
$\quad$ Männlich & 0,45 & 1,57 & $1,12-2,21$ & 1 \\
CHF & 0,43 & 1,53 & $1,16-2,03$ & 1 \\
IDDM & 0,58 & 1,79 & $1,18-2,72$ & 1 \\
Krea 1,2-2,0 & 0,78 & 2,18 & $1,51-3,14$ & 2 \\
$\quad$ Alter > 68 & 0,97 & 2,63 & $1,99-3,49$ & 2 \\
intraoperativ & & & & \\
HLM-Zeit > 134min & 0,67 & 1,95 & $1,46-2,60$ & 2 \\
EK > 1 & 0,72 & 2,05 & $1,51-2,79$ & 2 \\
FFP >0 & 1,2 & 3,32 & $1,2-8,59$ & 3 \\
\hline
\end{tabular}

\subsubsection{Diskriminationsfähigkeit des erweiterten Scores}

Durch die Berücksichtigung weiterer Faktoren konnte eine Verbesserung der Fläche unter der Kurve von ursprünglich 0,638 auf 0,773 erzielt werden, was einem hoch signifikantem Ergebnis entspricht $(p<0,0001)$. Die maximale Sensitivität $(63,29 \%)$ und Spezifität $(69,93 \%)$ wurde bei einem Cut-Off-Punkt > 4 Scorepunkten erreicht.

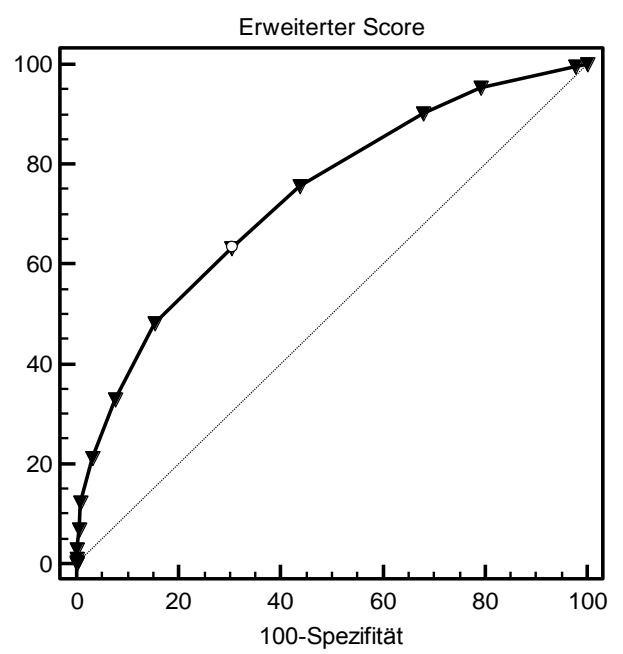

Abbildung 3.7 ROC-Kurve des erweiterten Scores 


\subsection{Zusammenfassung der Ergebnisse der Evaluierung und Vali- dierung}

Die Häufigkeitsverteilung der einzelnen Score-Werte stimmte im Göttinger Kollektiv gut mit den Original-Daten von Thakar überein. Die AKI-Inzidenz war im Göttinger Kollektiv sowohl für alle individuellen Score-Werte, als auch für die vier Score-Gruppen signifikant höher als im Cleveland-Kollektiv, sodass die Kalibrierung als unzureichend angesehen werden muss. Die Diskriminationsfähigkeit des Original-Scores war mit einer AUC von 0,638 in der ROC-Analyse signifikant geringer als innerhalb des Validierungskollektivs von Thakar. Durch eine Neuadjustierung der Score-Punkte für die einzelnen Risikofaktoren konnte die Diskriminationsfähigkeit nur minimal gesteigert werden, was in einer AUC von 0,653 resultierte $(p=0,53)$. Durch Hinzunahme von intraoperativen Risikofaktoren konnte die Diskriminationsfähigkeit des Scores dagegen statistisch signifikant gesteigert werden $(A \cup C=0,733, p<0,0001)$.

\subsection{Untersuchung der Auswirkung der Natriumbicarbonattherapie}

Der Effekt einer intra- und postoperativen Natriumbicarbonattherapie wurde an 448 Patienten untersucht. 224 Patienten erhielten diese Präventionsmaßnahme, $224 \mathrm{~Pa}$ tienten ohne Natriumbicarbonatgabe stellten das Kontrollkollektiv dar.

\subsubsection{Risikokonstellation}

Zur Überprüfung der Risikokonstellation der beiden Kollektive wurde präoperativ der Thakar-Score erhoben und intraoperativ die Konzentration des freien Hämoglobins, als vermutetes nephrotoxisches Agens, bestimmt.

\subsubsection{Scoreverteilung}

Die individuellen Scorewerte der Patienten lagen im Bereich zwischen 0 und 10 Punkten. Der mediane Scorewert betrug im Interventions- und Kontrollkollektiv 2 Punkte. 
Die Nullhypothese, dass die Scores in beiden Gruppen unterschiedlich verteilt sind, konnte mit Hilfe des Mann-Whitney-U-Tests mit der Irrtumswahrscheinlichkeit $p=0,6728$ sicher abgelehnt werden. Somit ist die Alternativhypothese anzunehmen, dass die individuellen Scores in beiden Gruppen gleich verteilt sind.

\subsubsection{Freie Serumhämoglobinwerte}

Der Median aller individuellen Mittelwerte der freien Serumhämoglobinwerte, die während der extrakorporalen Zirkulation mehrfach bestimmt wurden, betrug 27,00 mg/dl in der $\mathrm{NaHCO}_{3}$-Gruppe und $30,33 \mathrm{mg} / \mathrm{dl}$ in der Kontrollgruppe. Mittels des MannWhintney-U-Tests konnte festgestellt werden, dass die freien Serumhämoglobinwerte nicht signifikant $(p=0,0786)$ unterschiedlich in der $\mathrm{NaHCO}_{3}$-Gruppe und der Kontrollgruppe verteilt sind.

\subsubsection{Inzidenz der akuten Nierenschädigung}

Im Gesamtkollektiv von 448 Patienten konnte eine akute Nierenschädigung (AKIN 13) in 43,97 \% der Fälle nachgewiesen werden. Die Patienten, die eine präventive Therapie mit Natriumbicarbonat erhielten, zeigten eine Inzidenz von nur 37,5 \% im Gegensatz zur Kontrollgruppe, in welcher 50,45 \% unter einer akuten Nierenschädigung (AKIN 1-3) litten. Mittels $\mathrm{Chi}^{2}$-Test konnte das Ergebnis mit einer Irrtumswahrscheinlichkeit von $p=0,0007$ als hoch signifikant bewertet werden. Die unten stehende Abbildung verdeutlicht, dass in der $\mathrm{NaHCO}_{3}$-Gruppe die Anzahl an Patienten, die ein AKI entwickelten, deutlich kleiner ist als der Anteil an Patienten, die unter keinem AKI litten; wohingegen in der Kontrollgruppe der Anteil an Patienten, die kein AKI entwickelten, etwa genauso hoch ist wie der Anteil an Patienten mit AKI.

Tabelle 3.11 Vierfeldertafel AKI-Inzidenz

\begin{tabular}{c|cc} 
& $\mathrm{NaHCO}_{3}$-Gruppe & Kontrollgruppe \\
\hline AKI 0 & 140 & 111 \\
AKI 1-3 & 84 & 113
\end{tabular}




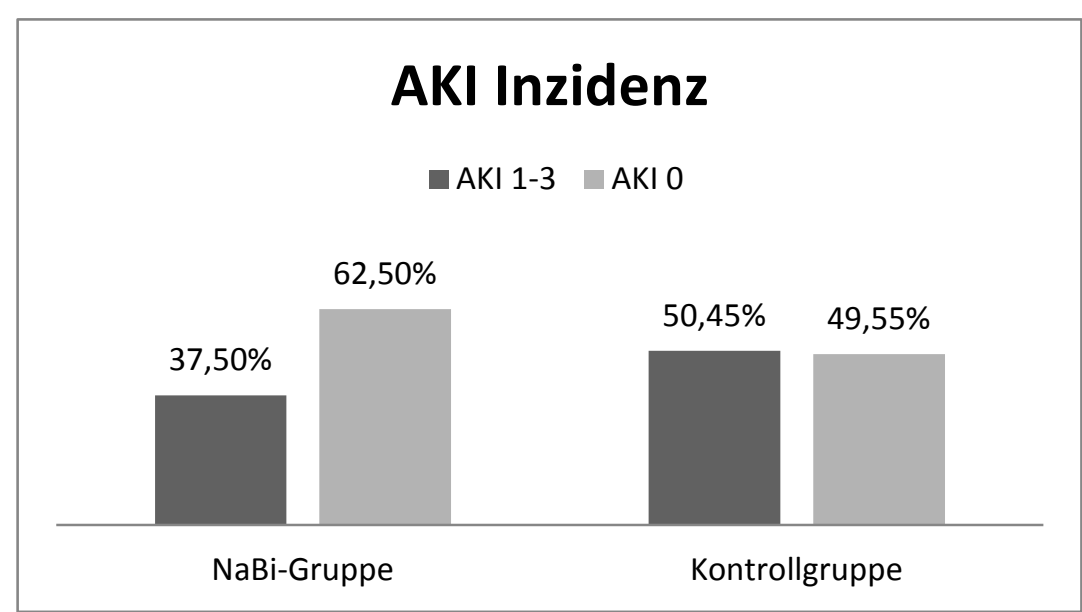

Abbildung 3.8 Histogramm AKI-Inzidenz 


\section{$4 \quad$ DISKUSSION}

\subsection{Relevanz der vorliegenden Studie}

Grundsätzlich ist die Erforschung des postoperativen akuten Nierenversagens weiterhin ein Thema von hoher Relevanz. Die postoperative akute Nierenschädigung nach herzchirurgischen Eingriffen unter Verwendung der Herz-Lungen-Maschine ist eine häufige und gravierende Komplikation (Conlon et al. 1999; Haase et al. 2007; Mangano et al. 1998; Parolari et al. 2012; Thakar et al. 2005). In bis zu 5 \% der Fälle kommen Nierenersatzverfahren zum Einsatz, ein leichter Anstieg des Kreatininwertes gegenüber dem präoperativen Ausgangswert findet sich bei der Hälfte der Patienten (Haase et al. 2007). Bereits geringe Veränderungen der Nierenfunktion sind mit einem allgemeinen schlechteren Outcome assoziiert und entscheidend für eine schlechte Prognose (Chertow et al. 2005). Die Gesamtmortalität nach Operationen mit HerzLungen-Maschinen liegt zwischen 2 und $8 \%$, bei Auftreten einer postoperativen akuten Nierenschädigung hingegen steigt die Mortalitätsrate exponentiell an (Bove et al. 2005; Thakar et al. 2005). Die akute Nierenschädigung ist allerdings nicht nur eine ernstzunehmende postoperative Komplikation, sondern bringt auch einen signifikant steigenden Kostenaufwand mit sich, insbesondere wenn der Einsatz eines Nierenersatzverfahrens notwendig wird (Mangano et al. 1998).

Die frühzeitige, idealerweise bereits präoperative Identifikation von Patienten mit erhöhtem Risiko einer akuten Nierenschädigung ist eine unabdingbare Voraussetzung für den gezielten Einsatz nephroprotektiver Maßnahmen. In der vorliegenden Arbeit wurde die Effektivität einer neuen Therapieoption - die Prävention mit Natriumbicarbonat - evaluiert; die Risikostratifizierung der Untersuchungskollektive erfolgte durch Anwendung des Thakar-Scores.

Bisher ist der Thakar-Score der am meisten validierte Score zur Abschätzung des Risikos akuter Nierenschädigungen nach kardiochirurgischen Eingriffen, unter anderem auch an einem Göttinger Kollektiv (Heise et al. 2010), der in den meisten EvaluierungsKollektiven auch eine hohen Diskriminationsfähigkeit vorwies (Huen und Parikh 2012).

Zwar existieren bereits diese diversen Studien zur Validierung des Thakar-Scores, dennoch unterliegen alle bisher publizierten Studien systematischen Schwachstellen, 
wie der Studiendurchführung in retrospektiver Form und der Definition des EndpunktKriteriums akutes Nierenversagen über die Anwendung einer Dialyse, die von individuellen Indikationsstellungen abhängig ist. So erfolgte diese Studie in prospektiver Form und unter Verwendung einer objektivierbaren, übertragbaren und präzisen Definition des AKI nach dem AKIN-Klassifikationssystem. Die vorliegende Studie stellt in dieser Hinsicht eine Neuheit dar.

\subsection{Design der Studie}

\subsubsection{Prospektives Studiendesign}

In bisherigen Validierungsstudien wurde die retrospektive Datenerhebung als Limitation der Aussagekraft der Studien angegeben. Es fiel auf, dass in retrospektiven Studien Daten oft nur unvollständig vorliegen, woraus eine Unschärfe bei der Bewertung der Risikofaktoren resultiert (Englberger et al. 2010; Heise et al. 2010; Huen und Parikh 2012). Diese Studie in prospektiver Form liefert dagegen auf Grund der vollen Verfügbarkeit aller Daten eine optimale Datenqualität. Fehlerquellen durch indirekte Einschätzungen bei mangelnder Verfügbarkeit von Daten konnten auf diese Weise verhindert werden (beispielsweise Erschließen von anamnestischen Angaben anhand der Vormedikation).

\subsubsection{Größe des Patientenkollektivs}

Um eine statistisch signifikante Aussage über die Validierung das Thakar-Scores machen zu können, ist eine gewisse Größe des Patientenkollektivs notwendig. Vergouwe et al. empfehlen auf Grund statistischer Analysen für die Größe eines externen Validierungskollektivs ein Minimum von 100 eintretenden Fällen des zu untersuchenden Endpunktes und 100 nicht-eintretende Fälle (Vergouwe et al. 2005). Auf Grund der Dialyse-Inzidenzen in Cleveland (Thakar et al. 2005) und Göttingen (Heise et al. 2010) sowie der angegebenen AKI-Inzidenz von Palomba et al. 2007 wurde eine voraussichtliche AKI-Inzidenz von mindestens 10 \% angenommen, sodass die Größe des Untersuchungskollektivs auf 1.000 festgelegt wurde, um mindestens 100 Fälle mit einer akuten Nierenschädigung zu erfassen. 
Die Abteilung Herzchirurgie des Universitätsklinikums Göttingen führt in einem Jahr etwa 1.000 Operationen unter der Verwendung der Herz-Lungen-Maschine durch, sodass ein Studienzeitraum von mindestens einem Jahr geplant wurde. Durch den prospektiven Charakter der Studie und die durch die Ethik-Kommission Göttingen geforderte Einwilligung der Patienten umfasste der Untersuchungszeitraum ein Jahr und acht Monate. Dies ist darauf zurückzuführen, dass einige Patienten wegen unvollständiger Datenerfassung und andere Patienten wegen mangelnder Einwilligungsfähigkeit oder -willen oder anderen Ausschlusskriterien (siehe Kapitel 2.3.2) nicht in die Studie eingeschlossen werden konnten.

Die primäre Validierung des Orginal-Scores, seine Modifikation und die anschließende Validierung wurden an demselben Kollektiv von 1.007 Göttinger Patienten durchgeführt. Für die Modifikation des Scores und seine Validierung wäre allerdings ein getrenntes Evaluierungs- und Validierungskollektiv wie in Cleveland optimal gewesen. Wäre das Kollektiv für die statistische Auswertung auf zwei Kohorten aufgeteilt worden, hätten bei $\mathrm{N}=500$ geringfügige Unterschiede von Risikofaktoren im Evaluierungskollektiv aber möglicherweise kein statistisches Signifikanzniveau erreicht. Deshalb wurde der Entschluss zur Evaluierung und Validierung am selben Kollektiv gefasst.

\subsubsection{Datenqualität}

Es ist zu beachten, dass die Beurteilung der einzelnen Risikofaktoren zur Ermittlung der individuellen Scorepunkte im Rahmen dieser prospektiven Studie deutlich präziser durchgeführt werden konnte und daraus Unterschiede in der RisikofaktorErfassung zwischen dieser Studie und Cleveland resultieren konnten. Um Datenunschärfen zu vermeiden, ist auf ein objektivierbares Vorgehen bei der Bewertung der Risikofaktoren (Beispiel Lungenfunktionstest statt Vormedikation zur Beurteilung einer COPD) oder sogar auf ausschließlich objektive Risikofaktoren an sich (linksventrikuläre Ejektionsfraktion statt subjektive Symptom-bestimmte NYHA-Klassifikation) zurückzugreifen.

Obwohl durch die prospektive Studienstruktur auf alle verfügbaren Daten uneingeschränkt zugegriffen werden konnte, bedurften einige wenige Parameter weiterhin der Interpretation. Beispielsweise auf den Risikofaktor „linksventrikuläre Ejektionsfrak- 
tion < $35 \%$ " musste bei fehlender konkreter prozentualer Angabe indirekt aus dem Textkontext „leichtgradig eingeschränkte EF“, „eingeschränkte EF“ und „schwer eingeschränkte EF“ geschlossen werden. Der letzte Fall wurde entsprechend einer „EF < $35 \%$ " gewertet und es wurde demzufolge ein Scorepunkt vergeben. Dies wurde einheitlich für alle Patienten so gehandhabt.

Zur bestmöglichen Eliminierung dieser interpretationsbedingten Unschärfe wurden alle Daten ausschließlich von einem Untersucher erhoben. So wurden die unvermeidbaren subjektiven Einschätzungen zumindest im gesamten Kollektiv unipersonal beziehungsweise monoperspektivisch ausgewertet und damit Interpretationsunschärfen minimiert.

\subsubsection{Endpunkt: Definition der akuten Nierenschädigung}

Heise et al. postulierten 2010, dass die vom Original-Score abweichenden Ergebnisse und die daraus resultierende mangelnde Übertragbarkeit der AKI-Inzidenzen daher begründet seien, dass die Definition des akuten Nierenversagens über die Anwendung eines Nierenersatzverfahrens erfolgte, deren Indikationsstellung zentrenabhängig an uneinheitliche Standards geknüpft ist und nicht übertragbar sei (Heise et al. 2010). So betonten Yallop und Smith weiterführend, dass sich solange keine einheitliche Definition des akuten Nierenversagens existiert, auch keine einheitlichen effektiven Therapiemaßnahmen zur Prävention des postoperativen ANV nach kardiochirurgischen Operationen etablieren können, da der Vergleich wissenschaftlicher Studien erschwert ist (Yallop und Smith 2004).

Man muss in diesem Zusammenhang erwähnen, dass zum Zeitpunkt der Entstehung des Thakar-Scores die RIFLE-Kriterien (Bellomo et al. 2004) gerade erst veröffentlicht wurden und die AKIN-Kriterien erst drei Jahre später entwickelt wurden (Mehta et al. 2007), sodass das von Thakar verwendete Kriterium Nierenersatzverfahren zum damaligen Zeitpunkt eine übliche Standard-Definition des akuten Nierenversagens darstellte. Inzwischen sind jedoch sowohl die RIFLE-Kriterien als auch die AKINKriterien etabliert (Kellum 2008), sodass konsequenterweise in dieser Studie die Definition des AKI anhand des aktuellen Standard-Klassifikationsystems - der AKINKlassifikation mit ihren einheitlichen, übertragbaren, praktikablen und vor allem präzi- 
sen Grenzwerten (Siehe Kapitel 1.1) - erfolgte. Eine Definition über die Anwendung einer Dialyse unterliegt nicht nur einer individuellen Indikationsstellung nach verschiedensten Standards als erhebliche systematische Fehlerquelle, sondern ist auch nicht mehr zeitgemäß.

Das in dieser Studie gewählte Endpunkt-Kriterium „AKI 0“ vs. „AKI 1-3“ entspricht demzufolge nicht dem Original-Endpunkt. Dies hat möglicherweise erhebliche Folgen auf die Kalibration und die Diskrimination. Da die AKIN-Kriterien jedoch das aktuelle Standard-Klassifikationssystem darstellen, sollte es folglich auch konsequent angewendet werden. Erkenntnisse über eventuelle Auswirkungen eines anderen EndpunktKriteriums auf die Anwendbarkeit des Thakar-Scores sind für die klinische Anwendung von großer Bedeutung. Nur hierdurch sind objektive Vergleiche mit anderen Zentren möglich.

Es erscheint sinnvoll, neben dem Thakar-Score den durch diesen an den aktuellen Standard angepassten modifizierten Thakar-Score in anderen Zentren zu evaluieren und zu validieren. Eine solche Studie wurde bereits in Kooperation zwischen der operativen Intensivstation des UMG Göttingen mit der operativen Intensivstation der Uniklinik Regensburg initiiert, die Ergebnisse stehen zum jetzigen Zeitpunkt noch aus.

\subsection{Ergebnisse der Studie}

\subsubsection{Häufigkeitsverteilung der Scorewerte}

Die im Göttinger Kollektiv erreichten Scorewerte lagen im Bereich 0 - 14 Punkte, wobei die Punkte 12, 13 und 15 - 17 nicht vergeben wurden. Im Original-Kollektiv in Cleveland erreichten die Patienten maximal 13 Punkte. Die prozentuale Verteilung der Scorepunkte zeigte sich in beiden Zentren sehr ähnlich. In dieser Studie und in Cleveland wurden die Scorewerte 2 und danach 3 am häufigsten vergeben. Nur die Verteilung der seltener vergebenen Scorewerte gestaltete sich etwas unterschiedlich. Dementsprechend ist davon auszugehen, dass die vorliegende Studie ein repräsentatives Patientenkollektiv darstellt, da eine ähnlich verteilte Ausprägung des patientenspezifischen Risikos wie in Cleveland festzustellen war; auch wenn das Göttinger Kollektiv nur 
1.007 Patienten umfasste im Gegensatz zu Thakar, der 15.838 Patienten in sein Evaluierungskollektiv einschloss.

\subsubsection{Häufigkeitsverteilung der Risikofaktoren}

Tabelle 4.1 Häufigkeitsverteilung der Risikofaktoren in Göttingen und Cleveland

\begin{tabular}{lccccl}
\hline Risikofaktor & \multicolumn{2}{c}{ Göttingen } & \multicolumn{2}{c}{ Cleveland } & P-Wert \\
& $\mathrm{N}$ & $\%$ & $\mathrm{~N}$ & $\%$ & \\
\hline CHF & 420 & 41,71 & 4221 & 26,65 & $\mathbf{P}<\mathbf{0 , 0 0 0 1}$ \\
Sonstige OP & 171 & 16,98 & 862 & 5,44 & $\mathbf{P}<\mathbf{0 , 0 0 0 1}$ \\
Re-OP & 53 & 5,26 & 3444 & 21,75 & $\mathbf{P}=\mathbf{0 , 0 0 6 3}$ \\
Klappe & 166 & 16,48 & 4068 & 25,69 & $\mathbf{P}=\mathbf{0 , 0 0 9 8}$ \\
Not-OP & 91 & 9,04 & 474 & 2,99 & $\mathbf{P}=\mathbf{0 , 0 1 5 7}$ \\
COPD & 148 & 14,70 & 1326 & 8,37 & $\mathbf{P}=\mathbf{0 , 0 1 6 4}$ \\
\hline IDDM & 140 & 13,90 & 1392 & 8,79 & $\mathrm{P}=0,0664$ \\
LVEF $<35$ & 119 & 11,82 & 1784 & 11,26 & $\mathrm{P}=0,0664$ \\
Weiblich & 259 & 25,72 & 4877 & 30,79 & $\mathrm{P}=0,0975$ \\
ACB + Klappe & 133 & 13,21 & 2594 & 16,38 & $\mathrm{P}=0,3973$ \\
IABP & 19 & 1,89 & 239 & 1,51 & $\mathrm{P}=0,4015$ \\
ACB & 537 & 53,33 & 8314 & 52,49 & $\mathrm{P}=0,739$ \\
\hline
\end{tabular}

Die Hälfte der Risikofaktoren war in Göttingen etwa gleich häufig vorhanden (beziehungsweise statistisch nicht signifikant unterschiedlich verteilt) wie in Cleveland. Hierzu zählten: „Anzahl der Aortokoronaren Bypass-Operationen“ (53,3 \% vs. 52,5 \%), „präoperative IABP“ (1,9\% vs. 1,5\%), „kombinierter ACB-Herzklappeneingriff“ (13,2 \% vs. $16,4 \%)$, „weibliches Geschlecht“ (25,7 \% vs. 30,8 \%), „linksventrikuläre Ejektionsfraktion“ (11,8\% und $11,3 \%)$ und „insulinpflichtigen Diabetes mellitus“ (13,9\% vs. $8,8 \%)$.

Die übrigen Risikofaktoren zeigten in Göttingen jedoch signifikant unterschiedliche Inzidenzen, wobei „Herzinsuffizienz", „sonstige Operation“, „Not-Operation“ und „COPD“ in Göttingen signifikant häufiger vorkamen und die Inzidenz der Risikofaktoren „Re-Operation“ und „Herzklappeneingriff“ signifikant niedriger war.

Für die Interpretation dieser Ergebnisse können diese Risikofaktoren in zwei Gruppen unterteilt werden: subjektive und objektive Risikofaktoren. 
Die ungleich verteilten Risikofaktoren „COPD“ und „Herzinsuffizienz" zählen zu den subjektiven Risikofaktoren. 41,7 \% der Patienten litten in Göttingen unter einer Herzinsuffizienz (NYHA III und NYHA IV), in Cleveland nur zwei Drittel davon (26,7\%). Hier ist anzumerken, dass die NYHA-Klassifikation eine rein symptomatische, schwer objektivierbare Einteilung darstellt, da sie auf subjektiven Patientenangaben basiert, die in Göttingen im Prämedikationsgespräch erfragt wurden. Diese Fehlerquote durch mangelnde Objektivierbarkeit wird bestätigt durch die im Gegensatz stehende objektive Einflussgröße „linksventrikuläre Ejektionsfraktion“, die erwartungsgemäß in Göttingen und Cleveland gleich verteilt war. Der Anteil der Patienten, die einen Scorepunkt für eine COPD erhielten, lag in Göttingen fast doppelt so hoch (14,7 \% vs. 8,4 \%). Dieser Unterschied ist auf die verschiedene Durchführung der Datenerhebung in den beiden Zentren zurückzuführen. Nur in Göttingen wurde dieser Risikofaktor objektiv erfasst. Thakar diagnostizierte eine COPD indirekt durch die Interpretation des Medikamentenplans mit einer COPD-Medikation der Patienten. Da die vorliegende Studie prospektiv erfolgte, konnten weitere Diagnoseschritte betrachtet werden, um bei unklarer Datenlage die Diagnose COPD zu stellen. Neben den Diagnoseaufzählungen in Arztbriefen und Überprüfung der Medikation wurde hier die präoperative Lungenfunktionsdiagnostik in Betracht gezogen, wonach eine Einteilung entsprechend des Gold-Schemas (siehe Anhang) erfolgte, sodass hierbei ein deutlich sensitiveres Verfahren gewählt wurde. In der vorliegenden Studie wurde die Bewertung des Risikofaktors COPD zwar objektiviert, wegen der mangelnden Anwendung von objektiven Diagnoseschemata im Erhebungskollektiv in Cleveland muss dieser Risikofaktor jedoch im Vergleich mit Cleveland weiterhin zu den subjektiven interpretationsbedürftigen Risikofaktoren gezählt werden.

Den objektiven Variablen lassen sich folgende Risikofaktoren unterordnen: „sonstige Operation“, „Herzklappeneingriff“, „Not-Operation“ und „Re-Operation“. Hierbei handelt es sich um absolut objektive Risikofaktoren, deren Erfassen keinerlei Interpretation erfordert. Die Inzidenz von „Sonstigen Operationen“ (17,0\% vs. 5,4\%) und „Not-Operationen“ (9,0\% vs. 3,0 \%) war in Göttingen eindeutig höher. Dagegen wurden in Cleveland ein vierfach höherer Anteil an "Re-Operationen“ (21,8\% vs. 5,3\%) und deutlich mehr „Herzklappenoperationen“ (25,7\% vs. $16,5 \%)$ durchgeführt. An dieser Stelle kommt den verschiedenen Zentren Bedeutung zu. Diese unterschiedli- 
chen Inzidenzen der Eingriffsvarianten sind wahrscheinlich dadurch begründet, dass die Indikationsstellungen für die Durchführung verschiedener Operationen zentrenspezifischen Unterschieden und Standards unterliegen.

Aus diesen charakteristischen Häufigkeitsverteilungen ergeben sich folgende Schlussfolgerungen: Auch wenn die Verteilung der Scorepunktesummen in Göttingen und Cleveland ähnlich waren, kamen einige relevante Risikofaktoren in sehr unterschiedlichen Ausprägungen vor. Diese Inhomogenität der Kollektive stellt eine Limitation bei der Übertragung von monozentrisch generierten Scores dar. Darüber hinaus lässt sich festhalten, dass subjektive, interpretationsbedürftige Daten die Anwendbarkeit und Gültigkeit eines Scores deutlich verschlechtern.

\subsubsection{Validität des Thakar-Scores}

Die Validitätsprüfung zeigte entgegen der Erwartung, dass trotz Optimierung des Studiendesigns mit prospektiver Datenerhebung und objektiver Endpunkt-Definition eine mangelhafte Kalibration und eine geringere Diskriminationsfähigkeit als im Original-Kollektiv von Thakar resultierten. Im Vergleich zu Validierungsstudien mit DialyseAnwendung als Endpunkt-Kriterium und retrospektiver Datenerhebung zeigte die vorliegende Arbeit schlechtere Ergebnisse (Candela-Toha et al. 2008; Heise et al. 2010).

\subsubsection{Kalibrierung}

Die Gesamtinzidenz des AKI lag in Göttingen um ein vielfaches höher als in Cleveland: $51,9 \%$ vs. 1,7\%. Dies ist begründet durch die deutlich verschiedenen EndpunktKriterien AKIN-Klassifikationssystem vs. Anwendung eines Nierenersatzverfahrens. Das AKIN-Klassifikationssystem, das bereits kleinste Nierenschädigungen mit geringsten Kreatinwert-Anstiegen berücksichtigt, stellt eine absolut sensitive Diagnose der akuten Nierenschädigung dar, während das Kriterium Nierenersatztherapie erst in der maximalen Ausprägung der akuten Nierenschädigung zutrifft; wobei Thakar darüber hinaus noch eine restriktive statt liberale Dialyse-Indikationsstellung vertrat, wodurch noch weniger Fälle tatsächlich das Kriterium der akuten Nierenschädigung erfüllten und ein so geringer Anteil von 1,7 \% zustande kam. 
In der vorliegenden Studie konnte gezeigt werden, dass die Höhe der individuell erreichten Scoresummen mit der Inzidenz einer akuten Nierenschädigung (AKIN 1 - 3) korrelieren. Je höher die Scoresumme war, desto höher war auch die AKI-Inzidenz. Weiterhin konnte gezeigt werden, dass auch die Inzidenz der kritischen AKIN-Klassen AKIN 2 und AKIN 3 mit steigendem Scorewert zunahm. Die AKI-Inzidenz der jeweiligen Scorewerte stimmte allerdings bei keinem Scorewert mit den Inzidenzen von Thakar überein. Demzufolge ist festzuhalten, dass der Thakar-Score im Göttinger Kollektiv keine gute Kalibrierung aufweist und damit keine präzise Vorhersagekraft besitzt, wenn als Endpunkt AKI >0 gewählt wird. Zu diesem Resultat der ungenügenden Kalibrierung kamen auch Heise et al. 2010 und die Arbeitsgruppe um Candela-Toha 2008. Auch Vives et al. konnten eine schlechte Kalibrierung des Scores mit einer tendenziellen Unterschätzung der Notwendigkeit eines Nierenersatzverfahrens feststellen, die besonders die Hoch-Risiko-Gruppen betraf (Vives et al. 2011). In einer Teil-Kohorte von Patienten mit sehr geringem Risiko konnte in einer anderen Studie eine gute Kalibrierung nachgewiesen werden (Candela-Toha et al. 2008).

\subsubsection{Diskrimination}

Die Diskriminationsfähigkeit des Thakar-Scores war im Göttinger Kollektiv deutlich geringer als in Cleveland. Die "Area under the curve" lag nur bei 0,638 im Gegensatz zu Cleveland mit 0,81 und 0,82 im Validierungskollektiv. Daraus resultierte eine nur mittelmäßige Sensitivität von 57,74 \% und Spezifität von 64,26 \%. Auch die Diskriminationsfähigkeit des Thakar-Scores kann dementsprechend nicht mit "gut" bewertet werden. Es wurden bereits mehrfach Studien veröffentlicht, die den Thakar-Score validierten. In einer spanischen (Candela-Toha et al. 2008), italienischen (Di Bella et al. 2007), US-amerikanischen (Englberger et al. 2010) und zwei kanadischen Kohorten (Wijeysundera et al. 2007) zeigte der Thakar-Score gute Ergebnisse mit einer AUC zwischen 0,80 und 0,86 . Auch Vives et al., die zwar eine mangelhafte Kalibration feststellten, berichteten von einer guten Diskriminationsfähigkeit des Scores zwischen HochRisiko- und Patienten mit geringem Risiko für ein AKI (Vives et al. 2011). Zwei Studien konnten eine sehr gute Trennschärfe mit einer AUC von 0,86 erzielen: eine mit einem sehr großen Kollektiv aus 12.096 Patienten (Englberger et al. 2010) und die zweite mit 
einem bedeutend kleineren Kollektiv von 1.780 Patienten (Candela-Toha et al. 2008), sodass nicht zwingend davon ausgegangen werden kann, dass die Größe des Kollektivs einen Einfluss auf die Trennschärfe hat. Interessanterweise konnten in Göttingen keine vergleichbaren Werte reproduziert werden, weder in der 2010 durchgeführten Validierungsstudie, in welcher der Wert der AUC nur geringgradig höher bei 0,66 lag (Heise et al. 2010), noch in der vorliegenden Untersuchung trotz Objektivitätsoptimierung.

\subsubsection{Ergebnisinterpretation}

Nachdem sowohl die Bewertung der Risikofaktoren durch die prospektive Form als auch die Definition des Outcome-Kriteriums mit Hilfe der standardisierten präzisen AKIN-Klassifikation maximal objektiviert wurden, ist eine Unschärfe und mangelnde Objektivität bei der Bewertung der individuellen Scorewerte als Ursache der nicht zufriedenstellenden Ergebnisse unwahrscheinlich. So ist davon auszugehen, dass diese Resultate höchst wahrscheinlich darin begründet sind, dass die primäre Entwicklung des Scores mit der Endpunkt-Definition der Nierenersatztherapie erfolgte, sodass die Anwendung des Scores, auch wenn - oder gerade weil - sie mit genaueren Definitionen durchgeführt wird, zu keinem besseren Ergebnis führt. Die Arbeitsgruppe um Englberger führte eine ähnliche Modifizierung des Endpunkt-Kriteriums durch, die zwar noch nicht alle Patienten erfasst, die dem AKIN-Stadium 1 entsprechen, aber den höheren AKIN-Stadien ähnelt. Die Endgröße wurde präzise als Serumkreatinin $>2,0 \mathrm{mg} / \mathrm{dl}$ und einer Verdopplung des präoperativen Kreatininwertes oder Einsatz einer Dialyse definiert. Im Gegensatz zu den Ergebnissen der vorliegenden Arbeit konnte der ThakarScore in ihrem Validierungskollektiv trotz Endpunkt-Modifikation interessanterweise weiterhin ein gutes Diskriminationsvermögen (AUC 0,81) aufzeigen (Englberger et al. 2010). Dieser Widerspruch gegenüber den Ergebnissen der vorliegenden Arbeit zeigt, dass nicht nur die identifizierten präoperativen Risikofaktoren Einfluss nehmen, sondern verdeutlicht die Relevanz zentrenspezifischer Unterschiede im intraoperativen und postoperativen Management. Möglicherweise ähneln sich die Standards in den Zentren der Arbeitsgruppen um Englberger und Thakar. Die eventuell in Göttingen existierenden maßgeblichen Risikofaktoren werden mit den 10 präoperativen Risikofaktoren des Thakar-Scores nicht ausreichend erfasst. So ist daraus abzuleiten, dass 
der Einfluss intraoperativer Faktoren wie Art und Dauer der HLM, Filtration und die Gabe von Blutprodukten sowie postoperative Standards der intensivmedizinischen Therapie wie diagnostische Kontrastmittelgabe und Antibiotikatherapie überprüft werden sollten. Speziell postoperative Risikofaktoren sind jedoch für die Voraussage eines AKI nur noch sehr bedingt geeignet, da sie erst zu einem vergleichsweise späten Zeitpunkt erfasst und gegebenenfalls modifiziert werden können.

Diese Ergebnisse der unzureichenden Kalibration und der geringeren Diskriminationsfähigkeit unterstreichen darüber hinaus die Notwendigkeit, bestehende Scores auf ihre "Anfälligkeit" gegenüber einer Objektivierung hinsichtlich der Bewertung der Risikofaktoren und vor allem hinsichtlich des Endpunkt-Kriteriums der Definition der akuten Nierenschädigung hin zu untersuchen. Scoring-Systeme sollten unbedingt auf möglichst eindeutigen, nicht-interpretationsbedürftigen Parametern basieren. Demzufolge erscheint es unerlässlich, modifizierte Scores zu entwickeln, in denen nur Einflusskriterien berücksichtigt werden, die Zentren-, Untersucher- und Patientenunabhängig objektiv bewertet werden können und ein Endpunkt-Kriterium beinhalten, das nicht nur einheitlich und objektiv ist, sondern auch den aktuellen Standards entspricht. Nur auf diese Art kann das tatsächliche Risiko postoperativer Nierenschädigungen evaluiert werden und nur dann können weitere Schritte der Entwicklung von Präventions- und Therapiemaßnahmen der postoperativen akuten Nierenschädigung evaluiert, angewendet und verbessert werden.

\subsubsection{Modifikation des Original-Scores}

\subsubsection{Neuberechnung des Original-Scores}

Die Neuberechnung der einzelnen Risiko-Punkte erbrachte keine erkennbare Steigerung der Diskriminationsfähigkeit.

Mittels uni- und multivariater Analyse wurde der signifikant unabhängige Einfluss der Risikofaktoren des Thakar-Scores auf das Endergebnis AKI im Göttinger Kollektiv untersucht. Es konnten fünf Parameter mit einem signifikanten unabhängigen Einfluss detektiert werden: „insulinpflichtiger Diabetes mellitus“, „Herzinsuffizienz“, „linksventrikuläre Ejektionsfraktion < $35 \%$ ", "Kreatininwert 1,2 - 2,0 mg/dl“ und "Art des Eingriffes" (kombinierter ACB-Herzklappeneingriff oder sonstige Operation). Für die 
Parameter "COPD“, „IABP“, „Not-Operation“, "Re-Operation“, „Herzklappeneingriff“ und „weibliches Geschlecht", die in den Thakar-Score einbezogen wurden, konnte kein unabhängiger signifikanter Einfluss gezeigt werden, wobei der Risikofaktor „Kreatininwert $\geq 2,1 \mathrm{mg} / \mathrm{dl}^{\prime \prime}$ an der Grenze des statistischen Signifikanzniveaus $(p=0,072)$ lag. Durch Vergrößerung des Patientenkollektives könnte dieser tendenzielle Unterschied der Verteilung in beiden Gruppen unter Umständen statistische Signifikanz erreichen. Der modifizierte Score des vorliegenden Kollektivs bestand daher nur aus fünf der ursprünglichen 10 präoperativen Risikofaktoren. Vergleichbare Resultate zeigten Di Bella et al. 2007, die an ihrem Validierungskollektiv ebenfalls nur für fünf der OriginalRisikofaktoren einen unabhängigen Einfluss nachweisen konnten.

Bei dem Vergleich der Punktegewichtung mit dem Thakar-Score aus Cleveland fiel auf, dass die Risikofaktoren „Herzinsuffizienz“, „linksventrikuläre Ejektionsfraktion $<35 \%$ " und "ACB-Herzklappeneingriff“ identische Scoresummen erhielten, die Variable „sonstige Operation“ wurde aber in der vorliegenden Analyse nur mit 1 statt 2 Punkten gewichtet, wohingegen der Risikofaktor „insulinpflichtiger Diabetes mellitus“ 2 statt nur 1 Punkt erhielt. Ein Kreatininwert zwischen 1,2 und 2,0 mg/dl wurde sogar mit 3 Scorepunkten gewichtet. Thakar vergab hierfür nur 2 Punkte, dagegen aber 5 Punkte für einen Kreatininwert $\geq 2,1 \mathrm{mg} / \mathrm{dl}$, der in diesem modifizierten Score auf Grund statistisch mangelnden Einflusses gar nicht vorkam.

Dennoch konnte auch die Anpassung der Gewichtung der einzelnen Risikofaktoren die Trennschärfe des Scores nicht deutlich verbessern. Der AUC-Wert erhöhte sich nur von 0,638 auf $0,652(p=0,53)$.

Die Beurteilungsstruktur der Risikofaktoren entspricht in der vorliegenden Studie optimaler Qualität und die Gewichtung der fünf Risikofaktoren wurde angepasst. Bei drei der fünf Risikofaktoren handelt es sich um objektive interpretationsfreie Risikofaktoren; nur die Risikofaktoren „Herzinsuffizienz“ und „insulinpflichtiger Diabetes mellitus" unterliegen eventuell einigen Interpretationsunschärfen. Dennoch konnte keine deutliche Verbesserung der Diskriminationsfähigkeit des Scores erzielt werden. Daraus lässt sich schließen, dass zentrenspezifische intra- und postoperative Standards einen vergleichsweise hohen Stellenwert im Einfluss auf ein AKI besitzen und dass die schlechteren Ergebnisse - verglichen mit dem Validierungskollektiv von Thakar - weniger an den unscharfen Definitionen der Risikofaktoren liegen, sondern vor allem an der 
überholten Definition des Endpunktes Nierenersatztherapie und der mangelhaften Übertragbarkeit des Thakar-Scores auf den präzisen AKIN-Endpunkt.

Daraus ergibt sich folgende Konsequenz für die Entwicklung zukünftiger Scores: Nur durch die primäre und konsequente Anwendung der AKIN-Klassifikation sowie eine objektive Bewertung der Risikofaktoren kann diese Fehlerquelle weitgehend eliminiert werden.

\subsubsection{Erweiterung um intraoperative Risikofaktoren}

In der Studie von Thakar wurden nur präoperative Einflussgrößen berücksichtigt (Thakar et al. 2005), in der vorliegenden Arbeit konnte jedoch darüber hinaus ein signifikanter Einfluss intraoperativer Faktoren auf die Entstehung einer akuten Nierenschädigung festgestellt werden. Für die Dauer eines Herz-Lungen-Maschinen-Einsatzes von mehr als 134 Minuten konnte ein signifikanter unabhängiger Einfluss nachgewiesen werden, wie bereits in anderen Studien berichtet wurde (Stallwood et al. 2004; Taniguchi et al. 2007). Dahingegen war die Aortenklemmzeit nicht als unabhängiger Risikofaktor an der Entstehung eines AKI beteiligt. Die Gabe von Blutprodukten, darunter die Gabe von Erythrozytenkonzentraten, aber vor allem die Gabe von Fresh-Frozen-Plasma zeigte in unserer Studie signifikante Auswirkungen, wobei der Einsatz von Thrombozytenkonzentraten keinen Einfluss nahm. Die Variable Filtration zeigte bereits in der monovariaten Analyse keine signifikant unterschiedliche Verteilung in den Gruppen AKI 0 und $\mathrm{AKI}>0$.

Parolari et al. beschrieben 2012, dass die Präzision von Scores verbessert werden kann, wenn zusätzlich intraoperative Variablen einbezogen werden. Der von ihnen entwickelte Score mit präoperativen Risikofaktoren konnte durch die Berücksichtigung intraoperativer Risikofaktoren eine Erhöhung der AUC von 0,83 auf 0,86 erzielen; durch zusätzlich postoperative Variablen sogar auf 0,9 (Parolari et al. 2012). In der vorliegenden Studie wurde der modifizierte Original-Score um drei intraoperative Risikofaktoren (HML-Dauer, EK, FFP) und darüber hinaus um eine präoperative Variable erweitert: Alter > 68 Jahre. Durch diese Erweiterung konnte die Fläche unter der ROCKurve auf 0,773 (95\% Kl: 0,705-0,760) vergrößert werden, im Gegensatz zu 0,638 
(95 \% KI: 0,608 - 0,668) bei Anwendung des Original-Scores. Dieser Unterschied war statistisch hoch signifikant $(p<0,0001)$.

Inwiefern die Kalibrierung des Scores durch die Modifizierung und Erweiterung verbessert werden kann, kann erst nach Anwendung auf das externe Kollektiv in Regensburg beurteilt werden.

Es ist festzuhalten, dass die Hinzunahme intraoperativer Risikofaktoren die Diskriminationsfähigkeit des Scores zwar signifikant verbesserte; doch wenn intraoperative Risikofaktoren mit erfasst werden, kann der Score folglich erst postoperativ errechnet werden und steht somit für die Planung des intraoperativen Vorgehens nicht mehr zur Verfügung. Vor dem Hintergrund einer frühestmöglichen Risikostratifizierung scheint daher die Begrenzung auf präoperative Faktoren ratsam zu sein. Dagegen kann im Rahmen von klinischen Studien die retrospektive Anwendung von umfangreicheren Scores bestehend aus prä- und intraoperativen Risikofaktoren zur präziseren Risikostratifizierung von Untersuchungskollektiven sinnvoll sein.

\subsubsection{Einfluss von Natriumbicarbonat auf die postoperative Nieren- funktion}

Bereits in den 80er Jahren wurde über präventive und therapeutische Effekte von Natriumbicarbonat diskutiert. Sporer et al. untersuchten 1981 die Wirkung von Natriumbicarbonat am Modell der Ratte, konnten damals jedoch keine Evidenz dafür finden, dass Natriumbicarbonat die renale Funktion im ischämischen Nierenversagen verbessert (Sporer et al. 1981). Atkins postulierte fünf Jahre später einen protektiven Effekt von Natriumbicarbonat (Atkins 1986).

Bis heute wurden verschiedene Studien und Metaanalysen veröffentlicht, die den Einsatz von Natriumbicarbonat untersuchten, jedoch nicht im Kontext des HerzLungen-Maschinen-Einsatzes. Die Ergebnisse der Studien waren sehr heterogen und ob die Gabe von Natriumbicarbonat tatsächlich eine effektive Therapie zur Prävention der akuten Nierenschädigung ist, blieb unklar (Atkins 1986; Kanbay et al. 2009; Merten et al. 2004; Sporer et al. 1981; Zoungas et al. 2009). Die Arbeitsgruppe um Haase et al. konnte jedoch 2009 mit einer randomisierten, doppelblinden, Placebo-kontrollierten Pilotstudie erstmals einen nephroprotektiven Effekt für Natriumbicarbonat bei kardio- 
chirurgischen Eingriffen unter extrakorporaler Zirkulation nachweisen (Haase et al. 2009).

In der vorliegenden Studie wurde nun untersucht, ob sich die Gabe von Natriumbicarbonat tatsächlich protektiv auf die Entwicklung eines AKI nach kardiochirurgischen Eingriffen auswirkt und ob damit die Ergebnisse der Pilotstudie von Haase et al. bestätigt werden konnten.

Zunächst musste die Vergleichbarkeit der beiden Patientenkollektive $\mathrm{NaHCO}_{3}-$ Gruppe und Kontrollgruppe hinsichtlich ihrer Scorewerte zur Beurteilung ihres Risikos zur Entwicklung eines AKI und hinsichtlich ihrer freien Serumhämoglobinwerte sichergestellt werden. Die Anwendung des Thakar-Scores mit Erhebung der individuellen Scorepunkte aller Patienten ermöglichte einen Vergleich der beiden Kollektive hinsichtlich der Summe ihrer patientenspezifischen Risikofaktoren. Bei gleich verteilten Scorewerten konnte von einer gleichen Risikoverteilung innerhalb der beiden Gruppen ausgegangen werden und die Gruppen waren hinsichtlich des Effekts von Natriumbicarbonat auf das Auftreten eines AKI vergleichbar.

Bei außerdem nahezu gleichmäßiger Verteilung der Konzentration an freiem Serumhämoglobin als vermutete nephrotoxische Substanz in beiden Gruppen konnte auch von einem vergleichbaren Ausmaß der HLM-abhängigen Hämolyse ausgegangen werden und ein Einfluss auf die Entstehung eines AKI durch eine höhere freie Hämoglobinkonzentration in der Kontrollgruppe ausgeschlossen werden.

Es zeigte sich in der vorliegenden Untersuchung eine hoch signifikant $(p<0,001)$ geringere Inzidenz einer akuter Nierenschädigung (AKIN 1 - 3) in der $\mathrm{NaHCO}_{3}$-Gruppe $(37,5 \%)$ als in der Kontrollgruppe $(50,45 \%)$ bei gleichen Score-Werten und nahezu gleichen Konzentrationen an freiem Serumhämoglobin in beiden Gruppen.

Demzufolge können diese Ergebnisse die Hypothese, dass die Natriumbicarbonatgabe einen präventiven Effekt auf die Entwicklung eines postoperativen akuten Nierenversagens besitzt, unterstützen. Natriumbicarbonat scheint die nephrotoxische Wirkung der HLM-abhängigen Hämolyse verhindern zu können. Die Ergebnisse der Pilotstudie von Haase et al. (Haase et al. 2009) konnten hiermit bestätigt werden. Es handelt sich um eine effektive, einfache, praktisch anwendbare und günstige präventive Therapiemaßnahme (Haase et al. 2007; Merten et al. 2004), deren Einsatz auf 
Grund der vorliegenden Datenlage empfohlen werden kann. Zur Zeit werden einige großangelegte internationale Phase-III-Studien durchgeführt (Haase et al. 2010).

Es ist zu erwähnen, dass die Größe des Untersuchungskollektivs von insgesamt 448 Patienten zwar größer war als in der Pilotstudie, die 100 Patienten umfasste, dennoch sollte die Veröffentlichung der Resultate der internationalen Studien mit großem Interesse verfolgt werden. Weiterhin handelt es sich bei der vorliegenden Studie nur um eine Beobachtungsstudie im Rahmen eines neu eingeführten klinischen Standardvorgehens ohne „echte“ Randomisierung: Nach Einführen eines neuen internen Standardvorgehens der operativen Intensivstation des Universitätsklinikums Göttingen, das basierend auf der Pilotstudie von Haase et al. (Haase et al. 2009) die intraoperative und 24-Stunden-postoperative Gabe von Natriumbicarbonat empfiehlt, erhielten alle Patienten diese präventive Maßnahme. Aus dieser Patientengruppe ergab sich das Kollektiv $\mathrm{NaHCO}_{3}$-Gruppe beziehungsweise die Interventionsgruppe. Die Kontrollgruppe bestand dagegen aus Patienten, die vor Einführung dieser standardisierten Gabe hospitalisiert waren und kein Natriumbicarbonat erhielten. Trotz der aufgeführten Einschränkungen konnte mit dieser Untersuchung der Effekt der NatriumbicarbonatPräventionsmaßnahme retrospektiv statistisch analysiert und anschließend bestätigt werden. So konnte damit der Forderung von Haase et al. nach Folge-Studien nachgekommen werden.

\subsection{Ausblick}

\subsubsection{Validierung anderer Scores, Vergleich von Scoring-Systemen}

Neben dem Thakar-Score existieren weitere Scoring-Systeme, die verschiedene Risikofaktoren einbeziehen, neben präoperativen auch intraoperative. Bisher scheint der Thakar-Score die größte Vorhersagekraft und die beste Diskriminationsfähigkeit zu besitzen (Englberger et al. 2010; Huen und Parikh 2012). Dennoch konnte noch keine Konsensentscheidung getroffen, oder gar eine Leitlinie veröffentlicht werden, die die Anwendung eines bestimmten Scores zur Risikostratifizierung eines AKI nach kardiochirurgischen Eingriffen empfiehlt (Huen und Parikh 2012). 
Mehta et al. publizierten 2006 einen Score, der an einem sehr großen multizentrischen Kollektiv aus 449.524 Patienten aus über 600 Krankenhäusern zwischen 2002 und 2004 entwickelt wurde. Diese große Anzahl beteiligter Zentren impliziert, dass systematische Fehler durch zentrenspezifische Einflüsse minimiert wurden. Es wurden identische Risikofaktoren wie in Cleveland bestimmt: präoperatives Serumkreatinin, Art des Eingriffes (Aortokoronarer Bypass mit Klappenoperation, alleinige Klappenoperation), Diabetes mellitus, NYHA-Stadium, Lungenerkrankung und Re-Operation. Darüber hinaus wurden noch vier weitere Risikofaktoren berücksichtigt: Alter, ethnische Rasse, Schock und kürzlich stattgehabter Myokardinfarkt. Auch hier wurde die Definition des ANV über die Anwendung eines postoperativen Nierenersatzverfahrens gestellt (Mehta et al. 2006). Trotz des großen multizentrischen Erhebungskollektivs konnte in Validierungsstudien gezeigt werden, dass der Thakar-Score eine bessere Diskriminationsfähigkeit aufweist (0,85 vs. 0,81) (Englberger et al. 2010). Dies könnte dadurch erklärt werden, dass der Mehta-Score ausschließlich für Patienten mit entweder einer ACB-Operation und/oder einem Aorten- oder Mitralklappeneingriff entwickelt wurde; wohingegen der Thakar-Score zur Prognose eines akuten Nierenversagens bei allen Eingriffen, die mit einer extrakorporalen Zirkulation einhergehen, uneingeschränkt angewendet werden kann. Zusammenfassend ließ sich feststellen, dass der ThakarScore die beste Diskrimination vorweist und darüber hinaus auch eine gute prognostische Aussagekraft besitzt, in einer Studie sogar dann, wenn objektive Endpunkte (wie beispielsweise Serumkreatinin) definiert und angewendet werden (Englberger et al. 2010).

\subsubsection{Scores mit Berücksichtigung der AKIN-Stadien}

Die bisher beschriebenen Scores prognostizieren lediglich die Wahrscheinlichkeit, mit der postoperativ ein Nierenersatzverfahren zur Anwendung kommt. Es konnte jedoch gezeigt werden, dass bereits geringe Veränderungen der Nierenfunktionen mit einem allgemein schlechteren Outcome assoziiert sind (Chertow et al. 2005; Lassnigg et al. 2008; Loef et al. 2005) und dass auch Patienten, die nur ein „mildes“ AKI entwickeln, das nicht dialysepflichtig ist, dennoch ein vierfach erhöhtes Risiko für kurzfristige und langfristige Mortalität im Vergleich zu Patienten mit uneingeschränkter Nieren- 
funktion nach kardiochirurgischen Eingriffen haben (Lok et al. 2004). Daraus geht hervor, dass es essentiell ist, den Fokus bereits auf geringe Veränderungen der Nierenfunktion (AKIN 1) zu legen und in Zukunft einen Score zu entwickeln und zu validieren, der nicht nur das terminale Nierenversagen, sondern auch das Risiko der weniger kritischen AKIN-Stadien präzise bewerten kann. Als Beispiel hierfür ist der AKICS-Score zu nennen (siehe Kapitel 1.7.3.2), der eine AKIN-ähnliche Definition als EndpunktKriterium nutzt: Serumkreatinin über $2,0 \mathrm{mg} / \mathrm{dl}$ oder ein Anstieg von über $50 \%$ zum Ausgangswert des Serumkreatinins (Palomba et al. 2007). Einschränkend ist jedoch zu erwähnen, dass neben präoperativen Risikofaktoren auch intra- und postoperative Variablen berücksichtigt werden, sodass eine frühzeitige präoperative Risikostratifizierung anhand dieses Scores nicht möglich ist. Obwohl der AKICS-Score bereits Anwendung zur Risikoabschätzung von Untersuchungskollektiven fand (Billings et al. 2011), existieren bis heute keine externen Validierungsstudien zu diesem Score.

\subsubsection{Anwendung und Auswirkung eines Scores}

Auffällig ist, dass sich die meisten Veröffentlichungen der Entwicklung von Vorhersagemodellen widmen. Eine kleinere Anzahl beschäftigt sich noch mit der Validierung der entwickelten Scores. Dagegen untersucht fast keine Studie mehr die Anwendung und Auswirkungen der Scores hinsichtlich ärztlichen Handelns oder PatientenOutcome (Reilly und Evans 2006).

Man kann zwischen verschieden Phasen der Forschungsarbeit an Vorhersagemodellen differenzieren (Justice et al. 1999).

- Phase $1 \quad$ Entwicklung

- Phase 2 Validierung

- Phase $3 \quad$ Auswirkung

- Phase 4 Realisierung

Phase eins stellt die eigentliche Entwicklung eines Scores dar, dies beinhaltet die Identifikation und Gewichtung von Risikofaktoren, interne Validierung mit Bestimmung der Vorhersagekraft und gegebenenfalls Optimierung des Scores. In der zweiten Phase 
wird ein Score extern validiert, was Thema dieser Arbeit war. Die Ergebnisse von Validierungen an anderen Zentren spielen eine zentrale Rolle in der Beurteilung von Scores, wobei die Übertragbarkeit getestet wird, das heißt, ob und wie präzise sich der Score auf andere Patientenkollektive anwenden lässt. Mit Hilfe der Daten aus den Validierungsstudien kann der Original-Score modifiziert und aktualisiert werden (Justice et al. 1999; Toll et al. 2008). In den darauffolgenden Phasen werden Nützlichkeit und Realisierung der Score-Anwendung evaluiert: Ist der Score auch praktikabel? Erfüllt ein Score seinen Zweck? Hilft er tatsächlich, ärztliche Entscheidungen zu treffen, zu klinischer Prozessoptimierung beizutragen, das Patienten-Outcome zu verbessern und können durch seine Anwendung Kosten reduziert werden (Toll et al. 2008)? Denn ohne die Anwendung und ohne eine Ableitung von klinischen Konsequenzen bleibt der Sinn von Scoring-Systemen limitiert.

Nachdem die Durchführbarkeit und auch der Nutzen eines Scores geprüft wurde, muss beobachtet werden, ob sich ein Score im klinischen Alltag schlussendlich auch etablieren kann. Diese letzte Phase wird als Realisierungsphase bezeichnet (Toll et al. 2008). Hierzu müssen in Zukunft weitere wissenschaftliche Studien publiziert werden und Vergleiche erfolgen. Erst dann können weiterführende Schritte folgen, wie beispielsweise die Entwicklung von Score-basierten Handlungsempfehlungen und Scoreadaptierten Therapie-Schemata. Darüber hinaus müssen Scores ständig auf ihre Aktualität hin geprüft und gegebenenfalls modifiziert werden.

Ein präzises und validiertes Vorhersagemodell für AKI nach herzchirurgischen Eingriffen muss also zum Ziel haben, eine Hilfe in der fundierten klinischen Entscheidungsfindung, in der Patientenberatung, im Einsatz klinischer Ressourcen und für die präoperative Optimierung ärztlichen Vorgehens darzustellen. Es ist wichtig, dass ein Score aus Variablen besteht, die einfach verfügbar und schnell zu berechnen sind, um die Patienten effektiv in klinische Studien einschließen zu können (Huen und Parikh 2012).

Mehta et al. bezeichneten ihren Score zur Identifizierung des Risikos für die postoperative Dialysepflichtigkeit bereits in der ersten Veröffentlichung als „BedsideTool“ (Mehta et al. 2006), was die einfache Anwendbarkeit des Scores impliziert. Doch ob der Score wirklich praktikabel oder einfach durchführbar ist und damit als „BedsideTool" bezeichnet werden kann und vor allem ob ein Score effektiv auch von Nutzen ist, 
kann erst durch multizentrische Evaluierung in klinischen Studien und anschließende weit verbreitete klinische Anwendung geklärt werden.

\subsubsection{Etablierte präventive Maßnahmen}

Zur Prävention eines postoperativen AKI existieren einige effektive Optionen. Dennoch sind die Möglichkeiten dieser effektiven nephroprotektiven Maßnahmen begrenzt und nicht alle universell einsetzbar. Geeignete Scores erlauben nicht nur deren gezielteren Einsatz, sondern bei guter Kalibrierung auch eine objektive Analyse ihres Effektes.

Idealerweise werden die Hoch-Risiko-Patienten bereits präoperativ identifiziert, sodass ein entsprechendes intraoperatives Vorgehen eingeleitet werden kann. Besonders für die Dauer des Herz-Lungen-Maschinen-Einsatzes konnte ein signifikanter unabhängiger Einfluss festgestellt werden, wie bereits in anderen Studien berichtet wurde (Stallwood et al. 2004; Taniguchi et al. 2007). Daraus ist abzuleiten, dass in Zukunft der Fokus auf der Verbesserung von intraoperativen chirurgischen und anästhesiologischen Verfahren zu legen ist. Die Anwendung der Herz-Lungen-Maschinen sollte optimiert werden, sowohl auf technischer Basis als auch durch zeitliche Begrenzung der HLM-Dauer oder sogar die Umstellung auf Off-pump-Techniken (Stallwood et al. 2004). Daneben stellt die Aufrechterhaltung des physiologischen Blutflusses und eines normwertigen arteriellen Mitteldruckes vor, während und nach der extrakorporalen Zirkulation einen wichtigen Punkt in der Prävention des postoperativen AKI dar (Haase et al. 2007).

Eine protektive Wirkung auf die Nierenfunktion scheint auch die Optimierung des intraoperativen Hämatokrits auf 21 - 25 \% (Karkouti et al. 2005) unter Vermeidung von massiver Hämodilution und daraus resultierend eine Aufrechterhaltung der Sauerstofftransportkapazität und Sauerstoffversorgung zu haben (Ranucci et al. 2005). Habib et al. konnten bereits bei einer Hämodilution mit einem Hämatokrit < $24 \%$ ein erhöhtes Risiko für postoperative Nierenschädigungen nachweisen (Habib et al. 2005).

Postoperativ können Scores als Entscheidungshilfe bei der Wahl der antibiotischen Therapie zum Einsatz kommen. So kann bei bekanntem AKI-Risiko der Einsatz nephrotoxischer Antibiotika wie Vancomycin vermieden werden und an dessen Stelle 
beispielsweise der Einsatz des weniger nephrotoxischen, aber teureren Reserveantibiotikums Linezolid gerechtfertigt werden. Weiterhin können mit Hilfe eines Scores Maßnahmen wie postoperative Kontrastmittelgabe fundierter diskutiert werden.

\subsubsection{Experimentelle präventive Maßnahmen}

In dieser Arbeit wurde über die Validierung (Phase 2) hinaus die Anwendung (Phase 3) des Thakar-Scores zur Untersuchung der Effektivität einer möglichen präventiven Therapie mit Natriumbicarbonat überprüft. In der $\mathrm{NaHCO}_{3}-\mathrm{Gruppe}$ zeigte sich eine signifikant geringere Inzidenz einer akuten Nierenschädigung als in der Kontrollgruppe. Demzufolge scheint Natriumbicarbonat die nephrotoxische Wirkung der HLMabhängigen Hämolyse vermindern zu können.

Neben der präventiven Gabe von Natriumbicarbonat existieren weitere vielversprechende Therapie-Aussichten zur Prävention der postoperativen akuten Nierenschädigung, deren vermutete Effekte in klinischen Human-Studien noch nachgewiesen werden müssen. Die Identifizierung gefährdeter Patienten dient dem zielgerichteten Einsatz präventiver Maßnahmen; der Nachweis pathophysiologischer Mechanismen ist jedoch essentiell, um weitere kausal-präventive Therapien überhaupt entwickeln zu können. Die Prävention und Therapie der intraoperativen hämodynamischen Auswirkungen und die Kontrolle der HLM-assoziierten Hämolyse stellen wesentliche Bereiche zukünftiger Studien dar (Haase und Shaw 2010).

\subsubsection{Haptoglobin}

Es konnte nachgewiesen werden, dass die nephrotoxischen Effekte von Hämproteinen durch die Gabe von Haptoglobin möglicherweise reduziert werden können, indem es freies Hämoglobin bindet und damit aus dem Kreislauf eliminiert. Die Plasmahaptoglobin-Konzentration sinkt sofort nach Beginn der HLM unter die Nachweisgrenze, sodass die bisher experimentelle Substitution von Haptoglobin während der extrakorporalen Zirkulation eine Strategie darstellen könnte, um Hämprotein-induzierte Nierenschädigung zu verhindern (Billings et al. 2011). 


\subsubsection{Caspase-Inhibitoren}

Cunningham et al. berichteten 2002, dass Apoptosemechanismen eine Schlüsselrolle in der Nierenschädigung nach hämodynamischen Veränderungen spielen, sowohl im Zustand eines hyperdynamen Kreislaufs bei Sepsis als auch durch die Reperfusion nach experimentellem ischämischen Zustand der Nieren (Cunningham et al. 2002). Caspase-3 repräsentiert in diesem Zusammenhang ein mögliches therapeutisches Konzept zur Unterdrückung von Apoptosemechanismen in den Nieren, die im letzten Schritt der proapoptotischen Signalprozesse in vielen Zellreihen aktiviert werden (Oyar et al. 2012). Die Apoptose könnte nun mit Caspase-Inhibitoren verhindert werden und damit einen protektiven und präventiven Effekt in Bezug auf die Nierenschädigung erzielen (Cunningham et al. 2002). Während der extrakorporalen Zirkulation kommt es zu hämodynamischen Veränderungen, insbesondere während der Aortenklemmzeit. Diese führt zu Koronarischämien, deren Reperfusion Auswirkungen auf den gesamten Organismus hat. Dementsprechend sollte eine Therapie mit Caspase-Inhibitoren experimentell auch in dieser Situation, die mit keiner direkten Ischämie der Nieren einhergeht, untersucht werden.

\subsubsection{Apotransferrin}

Während der Reperfusion ischämischer Organe wird redoxaktives Eisen in die systemische Zirkulation freigesetzt, dies konnte nach Transplantation von ischämisch geschädigten Organen nachgewiesen werden. Die Menge dieses zirkulierenden Eisens kann durch eine Behandlung mit Apotransferrin reduziert werden, sodass es zur Abnahme von oxidativem Stress und Entzündungsreaktionen kommt (de Vries et al. 2004). Die systemische Zirkulation des freien Eisens hat auch nephrotoxische Effekte zu Folge, wie es in Kapitel 1.5.3.2 (S. 18) erläutert wurde. Das intraoperative Abklemmen der Aorta führt zu einer vorübergehenden Koronarischämie, die auf die hier beschriebene ischämische Reperfusion übertragbar sein könnte. Wird die Menge des freien Eisens reduziert, könnte die Nierenschädigung verhindert werden. Demzufolge könnte auch eine Behandlung mit Apotransferrin eine Therapieoption des akuten Nierenversagens darstellen (de Vries et al. 2004). 


\subsubsection{Telmisartan}

Auch für Telmisartan, ein Angiotensin-II-Rezeptor-Antagonist, konnte im Tierversuch ein nephroprotektiver Effekt vor einer Nierenschädigung durch ischämische Reperfusion gezeigt werden. Für Telmisartan konnte eine antioxidative, antiinflammatorische und antiapoptotische Wirkung nachgewiesen werden, sodass es als potentielle Behandlungsmöglichkeit zur Prävention eines akuten Nierenversagens durch ischämische Reperfusion eine Rolle spielen könnte. Unbehandelte Ratten zeigten nach einer 45-minütigen Ischämie der Nieren mit anschließender Reperfusion für drei Stunden einen signifikant höheren Anstieg von Serumkreatinin, Stickstoffmonoxid, Malondialdehyd (ein Biomarker für oxidativen Stress), Blut-Harnstoff-Stickstoff, TNF $\alpha$ und eine erhöhte Aktivität von Caspase-3 (Fouad et al. 2010). Auch hier gilt es in Zukunft die Übertragbarkeit der direkten Nierenischämie auf die systemische Reperfusion nach ischämischem Zustand der Koronarien durch intraoperatives Abklemmen der Aorta zu klären. 


\section{$5 \quad$ Zusammenfassung}

Akute Nierenschädigung nach kardiochirurgischen Eingriffen ist eine häufige und ernstzunehmende Komplikation. Um präventive und therapeutische Maßnahmen zielgerichtet einsetzen zu können, ist die Risikostratifizierung der Patienten essentiell. Zu diesem Zweck wurden Scoring-Systeme entwickelt, die das individuelle Risiko für das Auftreten einer akuten Nierenschädigung abschätzen können. Geeignete Scores erlauben dann nicht nur den gezielteren Einsatz präventiver Maßnahmen, sondern auch eine objektive Analyse ihres Effektes. Der in dieser Arbeit validierte Thakar-Score mit dem EndpunktKriterium Nierenersatztherapie wies in den bisher durchgeführten Validierungsstudien eine gute Diskriminationsfähigkeit auf, die Kalibrierung schien jedoch nicht übereinstimmend gut zu sein. Seine Definition der akuten Nierenschädigung ist seit der Etablierung von objektivierbaren, vergleichbaren und präzisen Definitionen der RIFLE- oder AKINKriterien als überholt anzusehen. Erkenntnisse über die Auswirkungen eines anderen Endpunkt-Kriteriums auf die Anwendbarkeit des Thakar-Scores sind für die klinische Anwendung von großer Bedeutung. Nur hierdurch sind objektive Vergleiche mit anderen Zentren möglich. In der vorliegenden Arbeit wurde durch prospektives Studiendesign die Bewertung der Risikofaktoren optimiert und die den aktuellen Standards angepasste AKIN-Klassifikation als Endpunkt-Definition verwendet. Mit diesen Modifikationen konnten weder eine gute Kalibrierung noch eine gute Diskriminationsfähigkeit erreicht werden. Nur durch Hinzunahme intraoperativer Risikofaktoren konnte die Diskriminationsfähigkeit verbessert werden. Daraus ergibt sich, dass Scoring-Systeme auf möglichst eindeutigen, nicht-interpretationsbedürftigen Parametern basieren sollten und unbedingt ein Endpunkt-Kriterium beinhalten sollten, das nicht nur einheitlich und objektiv ist, sondern auch den aktuellen Standards entspricht. Nur dann können das tatsächliche Risiko postoperativer Nierenschädigungen evaluiert und ebenso weitere Schritte der Entwicklung von Präventions- und Therapiemaßnahmen der postoperativen akuten Nierenschädigung evaluiert, angewendet und verbessert werden.

Die praktische Anwendung des Scores zur Untersuchung des Effekts präventiver Therapiemöglichkeiten konnte in dieser Arbeit beispielhaft demonstriert werden. Anhand des Scores konnten gleiche Risikobedingungen in den Untersuchungskollektiven sicherge- 
stellt und ein direkter Effekt eines Therapieversuches evaluiert werden. Es wurde gezeigt, dass die Gabe von Natriumbicarbonat eine effektive Präventionsmaßnahme darstellt, um die Inzidenz der postoperativen akuten Nierenschädigung nach kardiochirurgischen Eingriffen zu reduzieren. 


\section{ANHANG}

\subsection{COPD Gold Schema}

\begin{tabular}{|c|c|c|c|c|}
\hline Schweregrad & I: leicht & II: mittel & III: schwer & IV: sehr schwer \\
\hline \multirow[t]{5}{*}{ Charakteristika } & $\begin{array}{l}\text { - } \mathrm{FEV}_{1} / \mathrm{VC}<70 \% \\
\cdot \mathrm{FEV}_{1} \geq 80 \% \\
\text { - mit/ohne } \\
\text { Symptomatik }\end{array}$ & $\begin{array}{l}\cdot \text { - } \mathrm{FEV}_{1} / \mathrm{VC}<70 \% \\
\cdot 50 \% \leq \mathrm{FEV}_{1}<80 \% \\
\cdot \text { mit/ohne } \\
\text { Symptomatik }\end{array}$ & $\begin{array}{l}\cdot \mathrm{FEV}_{1} / \mathrm{VC}<70 \% \\
\cdot 30 \%<\mathrm{FEV}_{1}<50 \% \\
\cdot \text { mit }_{\text {ohne }} \\
\text { Symptomatik }\end{array}$ & $\begin{array}{l}\cdot \mathrm{FEV}_{1} / \mathrm{VC}<70 \% \\
\cdot \mathrm{FEV}_{1} \leq 30 \% \text { oder } \\
\cdot \mathrm{FEV}_{1<50 \%} \text { und chronische } \\
\text { respiratorische Insuffizienz, } \\
\text { Zeichen der Rechtsherz- } \\
\text { insuffizienz }\end{array}$ \\
\hline & \multicolumn{4}{|c|}{$\begin{array}{l}\text { Vermeidung von Risikofaktoren, Grippe- und Pneumokokken-Schutzimpfung. } \\
\text { Zusätzlich bei Bedarf kurzwirksamer Bronchodilatator. }\end{array}$} \\
\hline & & \multicolumn{3}{|c|}{$\begin{array}{l}\text { Zusätzlich Dauertherapie mit einem oder mehreren langwirksamen } \\
\text { Bronchodilatatoren, Rehabilitation. }\end{array}$} \\
\hline & & & \multicolumn{2}{|c|}{$\begin{array}{l}\text { Zusätzlich inhalative Glukokortikoide bei } \\
\text { wiederkehrenden Exazerbationen. }\end{array}$} \\
\hline & & & & $\begin{array}{l}\text { Zusätzlich Langzeitsauerstoff- } \\
\text { therapie bei respiratorischer } \\
\text { Insuffizienz. } \\
\text { Prüfen, ob chirurgische } \\
\text { Behandlung angezeigt ist. }\end{array}$ \\
\hline
\end{tabular}

\subsection{Haber-Weiss-Reaktion}

$\cdot \mathrm{O}_{2}{ }^{-}+\mathrm{H}_{2} \mathrm{O}_{2} \rightarrow \bullet \mathrm{OH}+\mathrm{OH}^{-}+\mathrm{O}_{2}$

$\mathrm{Fe}^{2+}+\mathrm{H}_{2} \mathrm{O}_{2} \rightarrow \mathrm{Fe}^{3+}+\mathrm{OH}^{-}+\cdot \mathrm{OH}$

$\mathrm{Fe}^{3+}+\cdot \mathrm{O}_{2}^{-} \rightarrow \mathrm{Fe}^{2+}+\mathrm{O}_{2}$

$\bullet \mathrm{OH}=\mathrm{Hydroxyl}$-Radikale, $\mathrm{H}_{2} \mathrm{O}_{2}$ Wasserstoffperoxid, $\bullet \mathrm{O}_{2}{ }^{-}=$Superoxid 
ANHANG

-97- 
ANHANG 


\section{$7 \quad$ LITERATURVERZEICHNIS}

Abu-Omar Y, Ratnatunga C (2006): Cardiopulmonary bypass and renal injury. Perfusion $\underline{21}, 209-213$

Alpers C, Fogo A: The Kidney and Its Collecting System; in: Robbins basic pathology; hrsg. v. V Kumar AA, N Fausto, RN Mitchell 8. Auflage; Saunders Elsevier-Verlag, Philadelphia 2007, 541-578

Atkins JL (1986): Effect of sodium bicarbonate preloading on ischemic renal failure. Nephron $\underline{44}, 70-74$

Backhaus K, Erichson B, Plinke W, Weiber R: Multivariate Analysemethoden: eine anwendungsorientierte Einführung. 12. Auflage; Springer-Verlag, Berlin 2008

Bagshaw SM, Delaney A, Jones D, Ronco C, Bellomo R (2007): Diuretics in the management of acute kidney injury: a multinational survey. Contrib Nephrol 156, 236-249

Bahar I, Akgul A, Ozatik MA, Vural KM, Demirbag AE, Boran M, Tasdemir O (2005): Acute renal failure following open heart surgery: risk factors and prognosis. Perfusion 20 , 317-322

Barr LF, Kolodner K (2008): N-acetylcysteine and fenoldopam protect the renal function of patients with chronic renal insufficiency undergoing cardiac surgery. Crit Care Med $\underline{36}$, $1427-1435$

Bayati A, Nygren K, Kllskog O, Wolgast M (1990): The effect of loop diuretics on the long-term outcome of post-ischaemic acute renal failure in the rat. Acta Physiol Scand 139, 271-279

Bellomo R (2005): Management of early acute renal failure: focus on post-injury prevention. Curr Opin Crit Care 11, 542-547

Bellomo R, Ronco C, Kellum JA, Mehta RL, Palevsky P (2004): Acute renal failure - definition, outcome measures, animal models, fluid therapy and information technology needs: the Second International Consensus Conference of the Acute Dialysis Quality Initiative (ADQI) Group. Crit Care $\underline{8}$, R204-R212 
Bellomo R, Auriemma S, Fabbri A, D'Onofrio A, Katz N, McCullough PA, Ricci Z, Shaw A, Ronco C (2008): The pathophysiology of cardiac surgery-associated acute kidney injury (CSA-AKI). Int J Artif Organs $\underline{31}$, 166-178

Benedetto U, Angeloni E, Luciani R, Refice S, Stefanelli M, Comito C, Roscitano A, Sinatra R (2010): Acute kidney injury after coronary artery bypass grafting: does rhabdomyolysis play a role? J Thorac Cardiovasc Surg $\underline{140}$, 464-470

Billings FTt, Ball SK, Roberts LJ, 2nd, Pretorius M (2011): Postoperative acute kidney injury is associated with hemoglobinemia and an enhanced oxidative stress response. Free Radic Biol Med 50, 1480-1487

Bortz J: Statistik für Human- und Sozialwissenschaftler. 6. Auflage; Springer-MedizinVerlag, Berlin 2005

Bortz J, Schuster C: Statistik für Human- und Sozialwissenschaftler. 7. Auflage; SpringerMedizin-Verlag, Berlin 2010

Bourgeois E, Bataille A, Jacob L (2009): [Perioperative modifications in kidney function]. Press Med 38, 1621-1629

Bove T, Landoni G, Calabro MG, Aletti G, Marino G, Cerchierini E, Crescenzi G, Zangrillo A (2005): Renoprotective action of fenoldopam in high-risk patients undergoing cardiac surgery: a prospective, double-blind, randomized clinical trial. Circulation 111, 3230-3235

Candela-Toha A, Elias-Martin E, Abraira V, Tenorio MT, Parise D, de Pablo A, Centella T, Liano F (2008): Predicting acute renal failure after cardiac surgery: external validation of two new clinical scores. Clin J Am Soc Nephrol $\underline{3}$, 1260-1265

Chertow GM, Burdick E, Honour M, Bonventre JV, Bates DW (2005): Acute kidney injury, mortality, length of stay, and costs in hospitalized patients. J Am Soc Nephrol 16, 33653370

Coleman MD, Shaefi S, Sladen RN (2011): Preventing acute kidney injury after cardiac surgery. Curr Opin Anaesthesiol 24, 70-76

Conlon PJ, Stafford Smith M, White WD, Newman MF, King S, Winn MP, Landolfo K (1999): Acute renal failure following cardiac surgery. Nephrol Dial Transplant 14, 11581162 
Cunningham P, Dyanov H, Park P, Wang J, Newell K, Quigg R (2002): Acute renal failure in endotoxemia is caused by TNF acting directly on TNF receptor-1 in kidney. $\mathrm{J}$ Immunol $\underline{168}, 5817-5823$

Dasta JF, Kane-Gill SL, Durtschi AJ, Pathak DS, Kellum JA (2008): Costs and outcomes of acute kidney injury (AKI) following cardiac surgery. Nephrol Dial Transplant 23, 19701974

de-Mendona A, Vincent JL, Suter PM, Moreno R, Dearden NM, Antonelli M, Takala J, Sprung C, Cantraine F (2000): Acute renal failure in the ICU: risk factors and outcome evaluated by the SOFA score. Intensive Care Med 26, 915-921

de Vries B, Walter SJ, von Bonsdorff L, Wolfs TG, van Heurn LW, Parkkinen J, Buurman WA (2004): Reduction of circulating redox-active iron by apotransferrin protects against renal ischemia-reperfusion injury. Transplantation $\underline{77}, 669-675$

Di Bella I, Da Col U, Ciampichini R, Affronti A, Santucci A, Fabbri M, Sapia F, Ragni T (2007): [Validation of a new scoring system to predict the risk of postoperative acute renal failure in cardiac surgery]. G Ital Cardiol (Rome) $\underline{8}$, 306-310

Englberger L, Suri RM, Li Z, Dearani JA, Park SJ, Sundt TM, 3rd, Schaff HV (2010): Validation of clinical scores predicting severe acute kidney injury after cardiac surgery. Am J Kidney Dis $\underline{56}, 623-631$

Englberger L, Suri RM, Greason KL, Burkhart HM, Sundt TM, 3rd, Daly RC, Schaff HV (2011): Deep hypothermic circulatory arrest is not a risk factor for acute kidney injury in thoracic aortic surgery. J Thorac Cardiovasc Surg 141, 552-558

Falvo A, Horst HM, Rubinfeld I, Blyden D, Brandt MM, Jordan J, Faber MD, Silverman N (2008): Acute renal failure in cardiothoracic surgery patients: what is the best definition of this common and potent predictor of increased morbidity and mortality. Am J Surg 196, 379-383

Fouad AA, Qureshi HA, Al-Sultan AI, Yacoubi MT, Al-Melhim WN (2010): Nephroprotective effect of telmisartan in rats with ischemia/reperfusion renal injury. Pharmacology $\underline{85}, 158-167$

Friedrich J, Adhikari N, Herridge M, Beyene J (2005): Meta-analysis: low-dose dopamine increases urine output but does not prevent renal dysfunction or death. Ann Intern Med $\underline{142}, 510-524$

Haase M, Shaw A (2010): Acute kidney injury and cardiopulmonary bypass: special situation or same old problem? Contrib Nephrol 165, 33-38 
Haase M, Haase-Fielitz A, Bagshaw SM, Ronco C, Bellomo R (2007): Cardiopulmonary bypass-associated acute kidney injury: a pigment nephropathy? Contrib Nephrol 156, 340353

Haase M, Haase-Fielitz A, Bellomo R, Devarajan P, Story D, Matalanis G, Reade MC, Bagshaw SM, Seevanayagam N, Seevanayagam S, et al. (2009): Sodium bicarbonate to prevent increases in serum creatinine after cardiac surgery: a pilot double-blind, randomized controlled trial. Crit Care Med 37, 39-47

Haase M, Haase-Fielitz A, Bellomo R (2010): Cardiopulmonary bypass, hemolysis, free iron, acute kidney injury and the impact of bicarbonate. Contrib Nephrol 165, 28-32

Habib RH, Zacharias A, Schwann TA, Riordan CJ, Engoren M, Durham SJ, Shah A (2005): Role of hemodilutional anemia and transfusion during cardiopulmonary bypass in renal injury after coronary revascularization: implications on operative outcome. Crit Care Med 33, 1749-1756

Heise D, Sundermann D, Braeuer A, Quintel M (2010): Validation of a clinical score to determine the risk of acute renal failure after cardiac surgery. Eur J Cardiothorac Surg 37, $710-716$

Herold G: Innere Medizin 2012: eine vorlesungsorientierte Darstellung. Selbstverlag, Köln 2012

Heyman SN, Rosen S, Epstein FH, Spokes K, Brezis ML (1994): Loop diuretics reduce hypoxic damage to proximal tubules of the isolated perfused rat kidney. Kidney Int $\underline{45}$, 981-985

Hilgers R-D, Bauer P, Scheiber V, Heitmann KU: Einführung in die Medizinische Statistik; hrsg. v. Dette H, Härdle W; 2. Auflage; Springer-Verlag, Berlin 2007

Holmes CL, Walley KR (2003): Bad medicine: low-dose dopamine in the ICU. Chest $\underline{123}$, 1266-1275

Hou SH, Bushinsky DA, Wish JB, Cohen JJ, Harrington JT (1983): Hospital-acquired renal insufficiency: a prospective study. Am J Med $\underline{74}, 243-248$

Huber W, Eckel F, Hennig M, Rosenbrock H, Wacker A, Saur D, Sennefelder A, Hennico R, Schenk C, Meining A, et al. (2006): Prophylaxis of contrast material-induced nephropathy in patients in intensive care: acetylcysteine, theophylline, or both? A randomized study. Radiology 239, 793-804 
Huen SC, Parikh CR (2012): Predicting acute kidney injury after cardiac surgery: a systematic review. Ann Thorac Surg 93, 337-347

Jaenike JR (1967): The renal lesion associated with hemoglobinemia: a study of the pathogenesis of the excretory defect in the rat. J Clin Invest $\underline{46}, 378-387$

Joannidis M, Druml W, Forni LG, Groeneveld AB, Honore P, Oudemans-van Straaten HM, Ronco C, Schetz MR, Woittiez AJ (2010): Prevention of acute kidney injury and protection of renal function in the intensive care unit. Expert opinion of the Working Group for Nephrology, ESICM. Intensive Care Med 주, 392-411

Jones DR, Lee HT (2007): Protecting the kidney during critical illness. Curr Opin Anaesthesiol 20, 106-112

Justice AC, Covinsky KE, Berlin JA (1999): Assessing the generalizability of prognostic information. Ann Intern Med 130, 515-524

Kanbay M, Covic A, Coca S, Turgut F, Akcay A, Parikh C (2009): Sodium bicarbonate for the prevention of contrast-induced nephropathy: a meta-analysis of 17 randomized trials. Int Urol Nephrol 41, 617-627

Karkouti K, Beattie WS, Wijeysundera DN, Rao V, Chan C, Dattilo KM, Djaiani G, Ivanov J, Karski J, David TE (2005): Hemodilution during cardiopulmonary bypass is an independent risk factor for acute renal failure in adult cardiac surgery. $\mathrm{J}$ Thorac Cardiovasc Surg $\underline{129}, 391-400$

Keene WR, Jandl JH (1965): The sites of hemoglobin catabolism. Blood 26, 705-719

Kellum JA (2008): Defining and classifying AKI: one set of criteria. Nephrol Dial Transplant $\underline{23}, 1471-1472$

Kellum J, Leblanc M, Gibney RTN, Tumlin J, Lieberthal W, Ronco C (2005): Primary prevention of acute renal failure in the critically ill. Curr Opin Crit Care 11, 537-541

Krautzig S: Niere; in: Basislehrbuch Innere Medizin: kompakt - greifbar - verständlich; hrsg. v. Renz-Polster H, Krautzig S, Braun J; 3. Auflage; Elsevier, Urban \& FischerVerlag, München 2004, 878-951

Kubinger KD: Schlüsselbegriffe der psychologischen Diagnostik; hrsg. v. Jäger RS; 1. Auflage; Beltz-Verlag, Weinheim 2003 
Lameire N, Van Biesen W, Vanholder R (2005): Acute renal failure. Lancet $\underline{365}$, 417-430

Larsen R: Anästhesie. 9. Auflage; Elsevier, Urban \& Fischer-Verlag, München 2010

Lassnigg A, Schmid ER, Hiesmayr M, Falk C, Druml W, Bauer P, Schmidlin D (2008): Impact of minimal increases in serum creatinine on outcome in patients after cardiothoracic surgery: do we have to revise current definitions of acute renal failure? Crit Care Med $\underline{36}, 1129-1137$

Leone M, Martin C (2008): Vasopressor use in septic shock: an update. Curr Opin Anaesthesiol 21, 141-147

Leonhart R: Lehrbuch Statistik: Einstieg und Vertiefung. Hans Huber-Verlag, Bern 2004

Loef BG, Epema AH, Smilde TD, Henning RH, Ebels T, Navis G, Stegeman CA (2005): Immediate postoperative renal function deterioration in cardiac surgical patients predicts in-hospital mortality and long-term survival. J Am Soc Nephrol 16, 195-200

Lok CE, Austin PC, Wang H, Tu JV (2004): Impact of renal insufficiency on short- and long-term outcomes after cardiac surgery. Am Heart J 148, 430-438

Mack M, Pfister A, Bachand D, Emery R, Magee M, Connolly M, Subramanian V (2004): Comparison of coronary bypass surgery with and without cardiopulmonary bypass in patients with multivessel disease. J Thorac Cardiovasc Surg 127, 167-173

Mangano CM, Diamondstone LS, Ramsay JG, Aggarwal A, Herskowitz A, Mangano DT (1998): Renal dysfunction after myocardial revascularization: risk factors, adverse outcomes, and hospital resource utilization. The Multicenter Study of Perioperative Ischemia Research Group. Ann Intern Med 128, 194-203

Mason J, Kain H, Welsch J, Schnermann J (1981): The early phase of experimental acute renal failure. VI. The influence of furosemide. Pflugers Arch $\underline{392}, 125-133$

Mehta RH, Grab JD, O'Brien SM, Bridges CR, Gammie JS, Haan CK, Ferguson TB, Peterson ED (2006): Bedside tool for predicting the risk of postoperative dialysis in patients undergoing cardiac surgery. Circulation 114 , 2208-2216

Mehta RL, Kellum JA, Shah SV, Molitoris BA, Ronco C, Warnock DG, Levin A (2007): Acute Kidney Injury Network: report of an initiative to improve outcomes in acute kidney injury. Crit Care $\underline{11}, \mathrm{R} 31$ 
Merten GJ, Burgess WP, Gray LV, Holleman JH, Roush TS, Kowalchuk GJ, Bersin RM, Van Moore A, Simonton CA, 3rd, Rittase RA, et al. (2004): Prevention of contrast-induced nephropathy with sodium bicarbonate: a randomized controlled trial. JAMA $\underline{291}$, 23282334

Moosbrugger H: Testtheorie und Fragebogenkonstruktion; hrsg. v. Kelava A; SpringerVerlag, Berlin 2012

Mori Y, Sato N, Kobayashi Y, Ochiai R (2011): Acute kidney injury during aortic arch surgery under deep hypothermic circulatory arrest. J Anesth $\underline{25}$, 799-804

Nath KA, Norby SM (2000): Reactive oxygen species and acute renal failure. Am J Med $\underline{109}, 665-678$

Oyar EO, Kiris I, Gulmen S, Ceyhan BM, Cure MC, Delibas N, Lortlar N, Okutan H (2012): The protective effect of adrenomedullin on renal injury, in a model of abdominal aorta cross-clamping. Thorac Cardiovasc Surg $\underline{60}, 5-10$

Paller MS (1988): Hemoglobin- and myoglobin-induced acute renal failure in rats: role of iron in nephrotoxicity. Am J Physiol 255, F539-F544

Palomba H, de Castro I, Neto AL, Lage S, Yu L (2007): Acute kidney injury prediction following elective cardiac surgery: AKICS Score. Kidney Int 72, 624-631

Paparella D, Yau TM, Young E (2002): Cardiopulmonary bypass induced inflammation: pathophysiology and treatment. An update. Eur J Cardiothorac Surg 21, 232-244

Parolari A, Pesce L, Pacini D, Mazzanti V, Salis S, Sciacovelli C, Rossi F, Alamanni F (2012): Risk factors for perioperative acute kidney injury after adult cardiac surgery: role of perioperative management. Ann Thorac Surg 93, 584-591

Pospeschill M: Statistische Methoden: Strukturen, Grundlagen, Anwendungen in Psychologie und Sozialwissenschaften. 1. Auflage; Elsevier-Spektrum-Akademischer-Verlag, München 2006

Provenchere S, Plantefeve G, Hufnagel G, Vicaut E, De Vaumas C, Lecharny JB, Depoix JP, Vrtovsnik F, Desmonts JM, Philip I (2003): Renal dysfunction after cardiac surgery with normothermic cardiopulmonary bypass: incidence, risk factors, and effect on clinical outcome. Anesth Analg 96, 1258-1264 
Ranucci M, Romitti F, Isgro G, Cotza M, Brozzi S, Boncilli A, Ditta A (2005): Oxygen delivery during cardiopulmonary bypass and acute renal failure after coronary operations. Ann Thorac Surg 무, 2213-2220

Reilly BM, Evans AT (2006): Translating clinical research into clinical practice: impact of using prediction rules to make decisions. Ann Intern Med 144, 201-209

Riede U, Rumpelt H, Sauter G, Schmid O, Störkel S: Uropoetisches System; in: Allgemeine und spezielle Pathologie; hrsg. v. Riede U, Werner M, Schaefer H; 5. Auflage; ThiemeVerlag, Stuttgart 2004, 809-860

Roh GU, Lee JW, Nam SB, Lee J, Choi JR, Shim YH (2012): Incidence and Risk Factors of Acute Kidney Injury After Thoracic Aortic Surgery for Acute Dissection. Ann Thorac Surg $\underline{94}, 766-771$

Ronco C, Haapio M, House A, Anavekar N, Bellomo R (2008): Cardiorenal syndrome. J Am Coll Cardiol 52, 1527-1539

Sachs L, Hedderich J: Angewandte Statistik: Methodensammlung mit R. 13. Auflage; Springer-Verlag, Berlin 2009

Schrier RW, Wang W (2004): Acute renal failure and sepsis. N Engl J Med 351, 159-169

Silbernagl S: Die Funktion der Nieren; in: Physiologie; hrsg. v. Klinke R, Pape H-C, Silbernagl S; 5. Auflage; Thieme-Verlag, Stuttgart 2005, 325-376

Sisillo E, Ceriani R, Bortone F, Juliano G, Salvi L, Veglia F, Fiorentini C, Marenzi G (2008): $\mathrm{N}$-acetylcysteine for prevention of acute renal failure in patients with chronic renal insufficiency undergoing cardiac surgery: a prospective, randomized, clinical trial. Crit Care Med $\underline{36}, 81-86$

Skrabal C, Khosravi A, Westphal B, Steinhoff G, Liebold A (2006): Effects of poly-2methoxyethylacrylate (PMEA)-coating on CPB circuits. SCJ. Scand Cardiovasc J $\underline{40}$, 224229

Sporer H, Lang F, Oberleithner H, Greger R, Deetjen P (1981): Inefficacy of bicarbonate infusions on the course of postischaemic acute renal failure in the rat. Eur J Clin Invest $\underline{11}$, 311-315

Stallwood MI, Grayson AD, Mills K, Scawn ND (2004): Acute renal failure in coronary artery bypass surgery: independent effect of cardiopulmonary bypass. Ann Thorac Surg $\underline{77}$, 968-972 
Taniguchi F, de Souza A, Martins A (2007): Cardiopulmonary bypass time as a risk factor for acute renal failure. Rev Bras Cir Cardiovasc 22, 201-205

Thakar CV, Arrigain S, Worley S, Yared JP, Paganini EP (2005): A clinical score to predict acute renal failure after cardiac surgery. J Am Soc Nephrol 16, 162-168

Toll DB, Janssen KJ, Vergouwe Y, Moons KG (2008): Validation, updating and impact of clinical prediction rules: a review. J Clin Epidemiol 61, 1085-1094

Vergouwe Y, Steyerberg EW, Eijkemans MJ, Habbema JD (2005): Substantial effective sample sizes were required for external validation studies of predictive logistic regression models. J Clin Epidemiol 58, 475-483

Vermeulen Windsant I, Snoeijs M, Hanssen S, Altintas S, Heijmans J, Koeppel T, Schurink GWH, Buurman W, Jacobs M (2010): Hemolysis is associated with acute kidney injury during major aortic surgery. Kidney Int 77, 913-920

Vives M, Monedero P, Perez-Valdivieso JR, Garcia-Fernandez N, Lavilla J, Herreros J, Bes-Rastrollo M (2011): External validation and comparison of three scores to predict renal replacement therapy after cardiac surgery: a multicenter cohort. Int J Artif Organs 34 , 329-338

von Baeyer H: Pschyrembel Klinisches Wörterbuch 2012; hrsg. v. Pschyrembel W; 263. Auflage; de Gruyter-Verlag, Berlin 2011

Wijeysundera DN, Karkouti K, Dupuis JY, Rao V, Chan CT, Granton JT, Beattie WS (2007): Derivation and validation of a simplified predictive index for renal replacement therapy after cardiac surgery. JAMA $\underline{297}, 1801-1809$

Wilcox CS (1983): Regulation of renal blood flow by plasma chloride. J Clin Invest $\underline{71}$, 726-735

Yallop K, Smith D (2004): The incidence and pathogenesis of acute renal failure following cardiac surgery, and strategies for its prevention. Ann Card Anaesth $\underline{7}, 17-31$

Zager RA, Gamelin LM (1989): Pathogenetic mechanisms in experimental hemoglobinuric acute renal failure. Am J Physiol 256, F446-F455

Zanardo G, Michielon P, Paccagnella A, Rosi P, Cal M, Salandin V, Da Ros A, Michieletto F, Simini G (1994): Acute renal failure in the patient undergoing cardiac operation. Prevalence, mortality rate, and main risk factors. J Thorac Cardiovasc Surg 107, 14891495 
Zoungas S, Ninomiya T, Huxley R, Cass A, Jardine M, Gallagher M, Patel A, VasheghaniFarahani A, Sadigh G, Perkovic V (2009): Systematic review: sodium bicarbonate treatment regimens for the prevention of contrast-induced nephropathy. Ann Intern Med 151, 631-638 


\section{Danksagung}

Zunächst möchte ich Herrn Prof. Dr. med. Anselm Bräuer für die Ermöglichung dieser Dissertation und das Interesse an meiner Arbeit danken.

Mein besonderer Dank gilt Herrn Dr. med. Daniel Heise für seine großartige, uneingeschränkte und stetige Unterstützung bei der Durchführung dieser Arbeit, sein Engagement für die statistische Auswertung, seine konstruktiven Anregungen und Korrekturen und für seine ständige Hilfsbereitschaft bei vielen Fragen.

Weiterhin möchte ich den Mitarbeitern der Stationen 0118, 2012 und 2013 des Universitätsklinikums Göttingen für ihre freundliche Unterstützung während der Phase der Patienten-Rekrutierung danken. 